\title{
Hydrogen induced fast-fracture
}

\author{
Siamak S. Shishvan ${ }^{\mathrm{a}, \mathrm{b}}$, Gábor Csányi ${ }^{\mathrm{b}}$ and Vikram S. Deshpande ${ }^{\mathrm{b}^{*}}$ \\ ${ }^{a}$ Department of Structural Engineering, University of Tabriz, Tabriz, Iran. \\ ${ }^{\mathrm{b}}$ Department of Engineering, University of Cambridge, Cambridge CB2 1PZ, UK.
}

\begin{abstract}
One of the recurring anomalies in the hydrogen induced fracture of high strength steels is the apparent disconnect between their toughness and uniaxial tensile strength in identical hydrogen environments. Here we propose, supported by detailed atomistic and continuum calculations, that unlike macroscopic toughness, hydrogen-mediated tensile failure is a result of a fastfracture mechanism. Specifically, we show that failure originates from the fast propagation of cleavage cracks that initiate from cavities that form around inclusions such as carbide particles. The failure process occurs in two stages. In stage-A, hydrides rapidly form around the roots of stressed notches on the cavity surfaces with hydrogen fed from the hydrogen gas within the cavity. These hydrides promote cleavage fracture with the cracks propagating at $>100 \mathrm{~ms}^{-1}$ until the hydrogen gas in the cavity is exhausted. Predictions of this hydrogen-assisted crack growth mechanism are supported by atomistic calculations of binding energies, mobility barriers and molecular dynamics calculations of the fracture process. Typically, cracks grow by less than $1 \mu \mathrm{m}$ via this hydrogen-assisted mechanism and thus insufficient to cause macroscopic fracture of the specimen. However, this stage is then followed by a stage-B process where these fast propagating cracks can continue to grow, now in the absence of hydrogen supply, given an appropriate level of remote tensile stress. This is surprising because the fracture energy is now that of $\mathrm{Fe}$ in the absence of $\mathrm{H}$ and cleavage fracture requires opening tractions on the order of $15 \mathrm{GPa}$ to be generated. Thus, fracture is usually precluded due to plasticity around the crack-tip. Here we show via macroscopic continuum crack growth calculations in a rate dependent elastic-plastic solid with fracture modelled using a cohesive zone that cleavage is possible if the crack propagates fast enough. This is because strain-rates at the tips of fast propagating cracks are sufficiently high for the drag on the motion of dislocations resulting from phonon scattering to limit plasticity. This combined atomistic/continuum model is used to explain a host of well-established experimental observations including (but not limited to): (i) insensitivity of the strength to the concentration of trapped hydrogen; (ii) the extensive microcracking in addition to the final cleavage fracture event and (iii) the higher susceptibility of high strength steels to hydrogen embrittlement. Importantly, we also show that the stage-A hydrogen-assisted fracture process only occurs in certain crystallographic orientations with crack-tip plasticity processes, such as twinning, blunting cracks in other orientations. This inhibits the fast-fracture mechanism in a macroscopic toughness on a polycrystalline material and thus explains the apparent contradiction between the hydrogen-assisted macroscopic toughness and tensile strength of steels.
\end{abstract}

Keywords: Hydrogen embrittlement, Embedded Atom Model, fast-fracture.

*Corresponding author. E-mail address: vsd@eng.cam.ac.uk 


\section{Introduction}

When metallic alloys, including steels, are exposed to hydrogen they suffer a loss of tensile ductility and toughness leading to premature failure. Consequently, the adverse effects of hydrogen embrittlement must be included in engineering design for applications such as pipelines and nuclear power plants that come into contact with water, hydrocarbons or hydrogen gas. Hydrogen embrittlement is also critical for welded joints since hydrogen takeup can arise from the use of damp electrodes in electric welding operations.

While the adverse effects of hydrogen ingress on the mechanical properties of metallic alloys have long been recognized (Johnson, 1865; Pfeil, 1926) the mechanism of hydrogen embrittlement, especially in steels and ferrous alloys, remains a highly controversial issue with numerous mechanisms having been proposed; see the reviews by Lynch (2013) as well as Dadfarnia et al. (2015) and references therein. The two commonly invoked mechanisms are: (a) Hydrogen Enhanced DEcohesion (HEDE) and (b) Hydrogen Enhanced Localized Plasticity (HELP). According to the HEDE mechanism, hydrogen which has accumulated at a crack-tip reduces the cohesive strength giving rise to a reduced fracture toughness (Troiano, 1960; Oriani, 1972). By contrast, the HELP mechanism (Beachem, 1972; Birnbaum and Sofronis, 1994; Martin et al., 2019) assumes that hydrogen redistribution occurs around dislocations which in turn reduces the elastic interaction energy between dislocations and the associated reduction in Peierls stress results in material softening. However, there exist a number of experimental observations that are not only apparently inconsistent with each other but also cannot be rationalized by the commonly proposed embrittlement mechanisms. We proceed to discuss some of these observations in order to set the background of this study. For the sake of conciseness, we restrict the discussion to very low rates of loading where the observed embrittlement is most severe.

\subsection{Scaling of toughness and tensile strengths}

There is a large body of experimental data which suggests that while the tensile strength (and ductility) of notched and un-notched metallic alloy bars is significantly reduced in a hydrogen environment, the fracture toughness is less sensitive. Such observations have been reported for metals ranging from structural steels to nickel alloys and present a conundrum: the quasi-brittle fracture of the tensile bars is usually thought to result from the brittle propagation of small internal flaws in the presence of hydrogen (Song and Curtin, 2013) but the measured fracture toughnesses and tensile strengths in identical hydrogen environments seem inconsistent.

To quantify and expose this conundrum, we consider the strengths and toughnesses of a range of steels in a $69 \mathrm{MPa} \mathrm{H}_{2}$ atmosphere using the data summarised in San Marchi and Somerday (2012). The measured hydrogen-assisted mode-I elastic-plastic fracture toughness $J_{\mathrm{TH}}$ (i.e. the applied $J$ below which no crack growth occurs in the given hydrogen environment) is plotted as a function of their yield strength $\sigma_{\mathrm{Y}}$ in Fig. 1a. Also marked in Fig. 1a is the hydrogen environment embrittlement index (HEE index) for each alloy defined as the ratio of the tensile strength of the alloy in the $69 \mathrm{MPa} \mathrm{H}_{2}$ environment to the ultimate tensile strength (UTS) of the alloy in air. The $\mathrm{H}_{2}$ atmosphere reduces the tensile strength of all the alloys but the reduction is most significant for the high strength 4130 steel with a HEE of 0.4 . The commonly employed explanation for the reduction in tensile strength due to hydrogen charging, motivated by the HEDE mechanism, is as follows. Hydrogen reduces the toughness of the steels from $J_{\text {IC }}$ to the $J_{\mathrm{TH}}$ plotted in Fig. 1a. This hydrogen-assisted toughness is sufficiently low that small pre-existing flaws within the steels now propagate resulting in the premature fracture of the tensile specimens. For this argument to be valid, a $J$-field will need to exist around the preexisting cracks. The well-established criterion for the existence of a $J$-field (McMeeking and 
Parks, 1979) then specifies that a crack of size $a$ will only propagate in a hydrogen-assisted crack-like mode if

$$
a>a_{\mathrm{cr}}=50 \frac{J_{\mathrm{TH}}}{\sigma_{\mathrm{Y}}} .
$$

Contours of $a_{\mathrm{cr}}$ are included in Fig. 1a which suggest that these pre-existing flaws will need to be at least greater than $200 \mu \mathrm{m}$ in size and in some cases the flaws will need to be in the $\mathrm{mm}$ range for tensile fracture to be consistent with the measured $J_{\mathrm{TH}}$. Such large pre-existing flaws do not exist in these high-grade engineering steels. Nevertheless, brittle fracture with a fracture surface reminiscent of quasi-cleavage (Shibata et al., 2016; Nagao et al., 2012) is observed for the tensile failure of steels in a hydrogen environment. Thus, observations of hydrogen-assisted tensile fracture of steels seem to be inconsistent with fracture toughness measurements in identical hydrogen environments and the tensile failure mechanism remains unclear. Similar arguments can also be made for nickel alloys; see for example the data in Bechtle et al. (2009) for the toughness and tensile strength of Ni pre-charged with hydrogen.
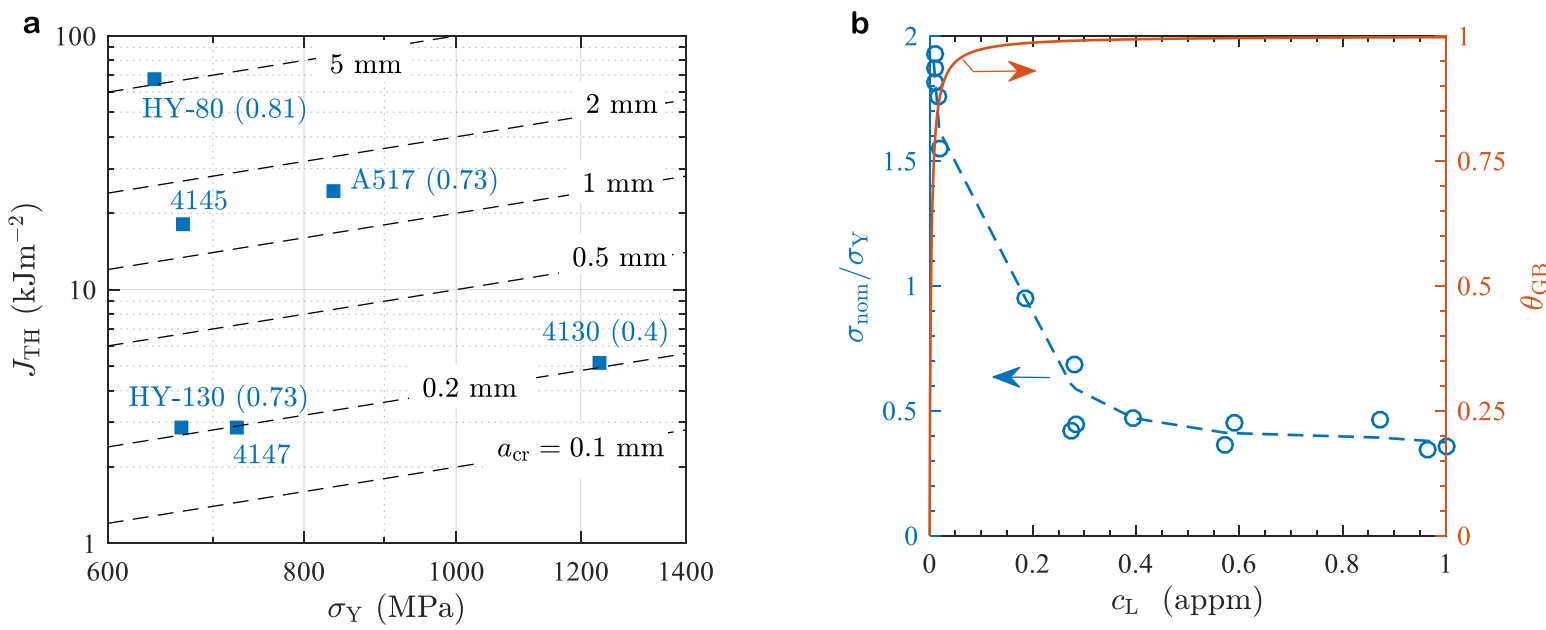

Figure 1: (a) Summary of the measured threshold toughness $J_{\mathrm{TH}}$ for a range of steels in a $69 \mathrm{MPa} \mathrm{H}_{2}$ atmosphere. The data is shown as a function of the yield strength $\sigma_{\mathrm{Y}}$ along with contours of the critical flaw size $a_{\mathrm{cr}}$ for the existence of a $J$-field. The corresponding hydrogen environment embrittlement (HEE) index defined as the ratio of the tensile fracture strength of the steel in a $69 \mathrm{MPa}_{2}$ atmosphere to the strength in air is also indicated (where available) alongside each data point. The data is taken from the technical reference compiled by San Marchi and Somerday (2012). (b) Summary of the nominal bending strength $\sigma_{\text {nom }}$ measurements of Novak et al. (2010) for a 4340 steel with yield strength $\sigma_{Y}=1500 \mathrm{MPa}$ as a function of the lattice hydrogen concentration $c_{\mathrm{L}}$. The corresponding Oriani (1972) predictions of the occupancy $\theta_{\mathrm{GB}}$ of grain boundary traps in 4340 steel are also included (righthand $y$-axis).

\subsection{The role of hydrogen traps}

The role of hydrogen trapping at microstructural defects upon hydrogen embrittlement also remains a controversial issue; see Gangloff (2003). For example, Novak et al. (2010) proposed a synergistic effect of HELP and HEDE as the mechanism of hydrogen embrittlement. They argued that the HELP mechanism reduces the length of pile-ups on carbides situated at the grain boundaries. Simultaneously, the presence of hydrogen reduces the interfacial cohesive strength of carbides on grain boundaries (HEDE) and this results in premature intergranular fracture. Thus, the view taken by Novak et al. (2010) and recently adapted by Nagao et al. (2018) is that the trapped hydrogen at dislocations and grain boundaries is critical to the embrittlement process. 
Observations of hydrogen embrittlement by intergranular cleavage (Wang et al., 2014) have also reinforced the view that hydrogen trapping at grain boundaries plays in critical role. However, there seems to be an inherent contradiction in this view as can be seen by examining the relation between the occupancy of grain boundary hydrogen sites and strength as recognised by Ayas et al. (2014). To exemplify this contraction, we include in Fig. 1b predictions of the occupancy $\theta_{\mathrm{GB}}\left(\equiv K \theta_{\mathrm{L}} /\left(1+K \theta_{\mathrm{L}}\right)\right.$ with $K=\exp \left(-\Delta H_{\mathrm{GB}} / R T\right), \Delta H_{\mathrm{GB}}$ the binding energy of grain boundary and $R$ the universal gas constant) of hydrogen binding sites at grain boundaries at a temperature $T=293 \mathrm{~K}$ as a function of the lattice hydrogen concentration $c_{\mathrm{L}} \equiv 10^{6} \theta_{\mathrm{L}}$ in appm (throughout this study we shall quote $c_{\mathrm{L}}$ in units of appm). These predictions are for the 4340 steel investigated by Novak et al. (2010) with $\Delta H_{\mathrm{GB}}=$ $-48 \mathrm{~kJ} \mathrm{~mol}^{-1}$ (throughout this manuscript all trap enthalpies/binding energies are quoted with respect to $\mathrm{H}$ in bulk unstressed $\mathrm{BCC} \mathrm{Fe}$ ) and clearly show that while the strength decreases gradually with increasing hydrogen concentration $c_{\mathrm{L}}>0.05 \mathrm{appm}$, this decrease is not correlated with the occupancy of grain boundary traps with $\theta_{\mathrm{GB}} \approx 1$ for $c_{\mathrm{L}}>0.05 \mathrm{appm}$. The fact that hydrogen in grain boundaries does not seem to play a major role is also supported by recent atomistic studies of the embrittlement of grain boundaries, albeit in Nickel, by Tehranchi and Curtin (2017a, 2017b).

A detailed assessment of the embrittlement of 4340 steels carried out by Ayas et al. (2014) confirmed that: (i) the level of hydrogen embrittlement scales with the lattice hydrogen and (ii) is independent of the hydrogen trapped in dislocations, grain boundaries or carbide particles. This is now widely recognized in the literature, i.e. the level of embrittlement scales with the diffusible (or equivalently lattice) hydrogen (Bhadeshia, 2016). In fact, reduction of the level of diffusible hydrogen by trapping of hydrogen at carbides (Yamasaki and Bhadeshia, 2006) and martensitic interfaces (Li et al., 2004) are being used as mechanisms to mitigate against embrittlement. With the experimental data suggesting that embrittlement in ferritic steels scales with lattice hydrogen that typically is less than $1 \mathrm{appm}$, the mechanism by which such low concentrations of hydrogen cause embrittlement remains unclear.

\subsection{Scope and outline of study}

The main aim of the current study is to present a coherent mechanism for understanding the dependence of the tensile strength of metallic alloys in a hydrogen environment and thereby rationalise the apparent contradictions discussed above. To limit the scope of the study we shall focus on steels and specifically present predictions using material data for 4340 steel even though we anticipate the proposed mechanism to be more widely applicable. Moreover, we shall restrict the analysis to the case of slow loading where embrittlement is most severe.

The outline of the paper is as follows. We first establish that fast crack propagation is essential for hydrogen-assisted cleavage fracture of $\mathrm{Fe}$ - this motivates the failure mechanism proposed here. Next, we give an overview of the proposed mechanism that involves a hydrogen-assisted fast-fracture mechanism initiating at the atomic scale but then evolving to result in macroscopic fracture. Based on this overview, we present a continuum model for hydrogen-assisted tensile fracture at a stress levels below the yield strength. The main assumptions of the atomic fracture processes employed in the continuum model are then justified via detailed atomistic calculations of the hydrogen ingress and fracture processes. The model is then extended to lower levels of hydrogen charging such that tensile fracture is accompanied by global plasticity. Finally, we end with further calculations to rationalise the high toughnesses measured in traditional fracture toughness tests and importantly provide model predictions for loading cases where no experimental data exists to-date - such a priori predictions can form the basis for the critical future validation of the proposed hydrogen-assisted fracture mechanism. 


\section{Motivation and overview of the hydrogen embrittlement model}

The observations discussed above suggest that a hydrogen-assisted cleavage crack propagates under tensile loading at stress levels far below than that estimated from toughness measured in an identical hydrogen environment. To understand this apparent contradiction and motivate our proposed mechanism we first establish a key requirement to be met for a crack to propagate by hydrogen-assisted cleavage.

Detailed atomistic calculations for the effect of hydrogen on a mode-I loaded crack with a crack plane (110) and crack front direction [110] in BCC Fe are presented in Section 4 (these predictions are broadly consistent with those of Song and Curtin (2013) for cracks on the (111) plane). They demonstrate that loading enables hydrides of size $\ell_{\mathrm{H}} \approx 1.25 \mathrm{~nm}$ to form at sharp crack tips and these hydrides promote cleavage fracture at an applied mode-I stress intensity factor $K_{\mathrm{I}}=K_{\text {cleavage }}^{\mathrm{H}} \approx 1 \mathrm{MPa} \sqrt{\mathrm{m}}$. However, immediately after cleavage there is no hydride at the crack tip as the hydrogen in the hydride is consumed to cover the newly formed fracture surface. In the absence of a crack-tip hydride, the barrier for dislocation emission is also vanishingly small at $K_{\mathrm{I}} \approx 1 \mathrm{MPa} \sqrt{\mathrm{m}}$, i.e. $K_{\text {emit }} \approx 1 \mathrm{MPa} \sqrt{\mathrm{m}}$, and therefore dislocation emission is expected to ensue from such a crack tip. If hydrogen cannot be supplied to sufficiently rapidly replenish the crack tip hydride, significant blunting due to dislocation emission will ensue at $K_{\mathrm{I}}=1 \mathrm{MPa} \sqrt{\mathrm{m}}$ and further cleavage will be precluded; see sketches in Fig. 2a. Thus, there is a direct competition between the rate of formation of a crack-tip hydride (and consequently the rate of crack propagation by cleavage) and crack blunting by dislocation emission.

An estimate of the minimum crack velocity $v_{\text {blunt }}^{\text {crit }}$ required for preventing blunting by dislocation emission requires two ingredients: (i) a definition of a blunt crack, i.e. the number $\alpha$ of dislocations emitted to blunt a sharp crack and reduce stress levels to preclude cleavage and (ii) the number $\mathcal{N} \equiv \dot{\mathcal{N}} \mathcal{A} / v_{\text {c }}$ of dislocations emitted from the crack tip travelling at a speed $v_{\mathrm{c}}$ in the time it needs to propagate one atomic spacing $\mathcal{A}$ with $\dot{\mathcal{N}}$ denoting the rate of dislocation emission. The minimum velocity $v_{\mathrm{c}}=v_{\text {blunt }}^{\text {crit }}$ required for cleavage is defined by the condition $\mathcal{N}<\alpha$. The level of blunting parametrised by the number $\alpha$ of dislocations that a crack tip can emit but yet propagate by cleavage follows from the requirement that the cleavage stress $T_{\max }=15 \mathrm{GPa}$ needs to be attained at the notch (i.e. blunt crack) root at a remote applied $K_{\mathrm{I}}=1 \mathrm{MPa} \sqrt{\mathrm{m}}$. On the other hand, the rate of dislocation emission $\dot{\mathcal{N}}$ can be calculated by recognising that an emitted dislocation shields the crack tip and prevents emission until it has travelled a sufficient distance from the crack tip that the crack tip stressintensity recovers to $K_{\text {emit }}$.

A detailed analysis based on these general principles is presented in Appendix A with predictions of $v_{\text {blunt }}^{\text {crit }}$ are included in Fig. $2 \mathrm{~b}$ as a function of $K_{\mathrm{I}} / K_{\mathrm{emit}}$ for parameters representative of Fe (included in Table 1). To put these results in context, consider the case of a centre-cracked structure with a crack of half-length $\lambda$ subjected to a remote tensile stress $\sigma$. Recall that cleavage crack extension commences at $K_{\mathrm{I}}=\sigma \sqrt{\pi \lambda}=1 \mathrm{MPa} \sqrt{\mathrm{m}}$ with the stress intensity increasing to $K_{\mathrm{I}}=\sigma \sqrt{\pi(\lambda+\Delta \lambda)}$ after the crack has extended by $\Delta \lambda$. We then rewrite $K_{\mathrm{I}} / K_{\text {emit }}$ in terms of crack extension and include in Fig. $2 \mathrm{~b}$ a double abscissa to also quantify $v_{\text {blunt }}^{\text {crit }}$ as a function of the normalised crack extension $\Delta \lambda / \lambda$. The key result is that a cleavage crack velocity $v_{\mathrm{c}}>v_{\text {blunt }}^{\text {crit }} \approx 5.4 \mathrm{~ms}^{-1}$ is required to frustrate blunting of the crack-tip by dislocation emission and allow any reasonable level of crack extension. Below a velocity 
$v_{\text {blunt }}^{\text {crit }}$, the crack is expected to arrest by blunting due to dislocation emission after minimal extension.

a

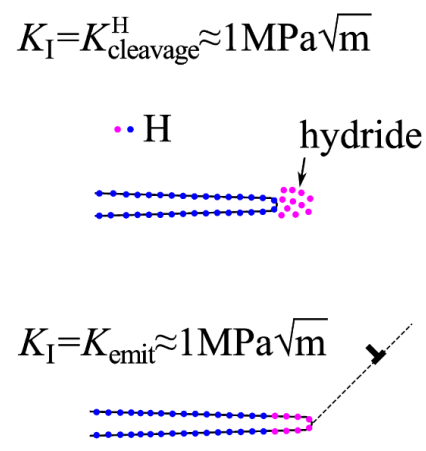

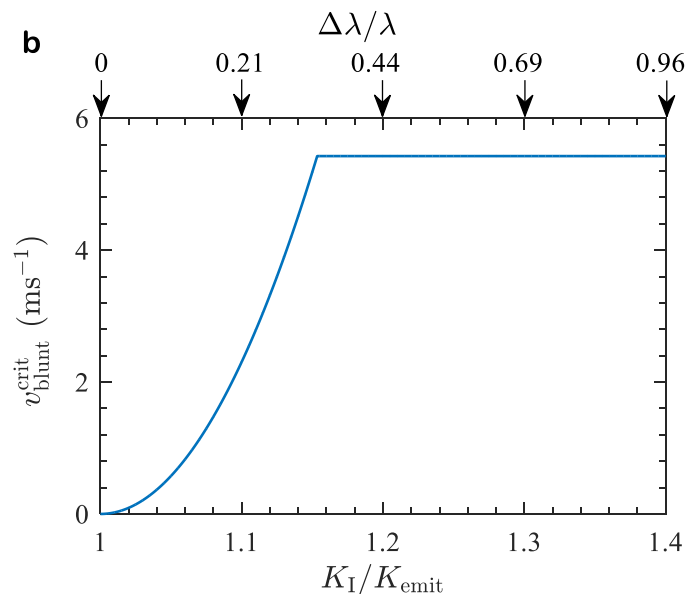

Figure 2: (a) Sketches showing the cleavage of a pre-existing crack-tip hydride (with hydrogen within the hydride covering the newly formed fracture surfaces) followed by the subsequent emission of dislocations from the crack-tip. Cleavage can only recommence if the hydride reforms before blunting the crack-tip by dislocation emission. (b) Predictions of the critical velocity $v_{\text {blunt }}^{\text {crit }}$ to ensure propagation of a crack with minimal blunting due to dislocation emission. This velocity is plotted as function of the applied mode-I stress intensity factor $K_{\mathrm{I}}$ normalised by the stress intensity factor $K_{\text {emit }}=1 \mathrm{MPa} \sqrt{\mathrm{m}}$ for dislocation emission. The double abscissa shows the corresponding normalised crack extension $\Delta \lambda / \lambda$, where $\lambda$ is the half-length of the initial crack.

\subsection{Cleavage crack speed governed by diffusive hydrogen flux from lattice}

Cleavage can occur only due to the presence of a crack-tip hydride and the hydrogen required to form this hydride is usually assumed to be supplied via diffusive flux from the specimen bulk to a loaded crack-tip; see for example Sofronis and McMeeking (1989) and Song and Curtin (2013). The speed of the cleavage crack is thus limited by the diffusive flux rate of $\mathrm{H}$ driven by concentration gradients that develop as hydrogen from the lattice covers sites on the newly formed fracture surfaces. This speed can be readily estimated and here we provide a simple analytical estimate (it differs somewhat from the Song and Curtin (2013) estimate that included the crack-tip stress gradients). Consider the growth in the radius of a penny-shaped crack from $\zeta$ to $\zeta+\Delta \zeta$ in time $\Delta t$ due to hydrogen-assisted cleavage. Given that the binding affinity of surface $\mathrm{H}$ sites $\Delta H \approx-96 \mathrm{~kJ} \mathrm{~mol}^{-1}$ (Jiang and Carter, 2004a) all $\mathrm{H}$ binding sites on the newly formed surfaces will require to be occupied by the $\mathrm{H}$ supplied by diffusion under equilibrium conditions. In time $\Delta t$, the diffusion distance of $\mathrm{H}$ is $\approx \sqrt{D_{\mathrm{L}} \Delta t}$, where

$$
D_{\mathrm{L}}=D_{0} \exp \left(-\frac{Q_{\mathrm{D}}}{R T}\right) \text {, }
$$

is the lattice diffusion co-efficient for $\mathrm{H}$ in BCC Fe, $D_{0}=2.1 \times 10^{-7} \mathrm{~m}^{2} \mathrm{~s}^{-1}$ is the preexponential diffusion factor and $Q_{\mathrm{D}}=6.6 \mathrm{~kJ} \mathrm{~mol}^{-1}$ the lattice activation energy for diffusion in bulk unstressed 4340 steel (Nelson and Stein, 1973). Thus, H required to cover the newly formed surface is obtained from within a toroidal region with radius of revolution $\zeta$ and a circular section of radius $\approx \sqrt{D_{\mathrm{L}} \Delta t}$ located at the crack front. Mass balance of $\mathrm{H}$ then requires

$$
2 \pi^{2} \zeta \theta_{\mathrm{L}} \rho_{\mathrm{m}}\left(\sqrt{D_{\mathrm{L}} \Delta t}\right)^{2}=2 \Gamma_{\mathrm{s}}(2 \pi \zeta) \Delta \zeta
$$


where $\rho_{\mathrm{m}} \equiv \rho / M$ is the molar density of $\mathrm{Fe}$ in terms of the density $\rho$ of $\mathrm{Fe}$ and the molar mass $M$ while $\Gamma_{\mathrm{s}}=2.65 \times 10^{-5} \mathrm{~mol} \mathrm{~m}^{-2}$ (Wang and Vehoff, 1991) is the areal density of $\mathrm{H}$ binding sites on the Fe surface. The crack front velocity then follows as

$$
v_{\mathrm{d}}^{\text {crit }} \equiv \frac{\Delta \zeta}{\Delta t}=\frac{\pi D_{\mathrm{L}} \theta_{\mathrm{L}} \rho_{\mathrm{m}}}{2 \Gamma_{\mathrm{s}}}
$$

Using the values from Table 1, we see that even for a very high level of hydrogen charging corresponding to $c_{\mathrm{L}}=10^{6} \theta_{\mathrm{L}}=1 \mathrm{appm}, v_{\mathrm{d}}^{\text {crit }} \approx 120 \mu \mathrm{m} \mathrm{s}^{-1}$ at a temperature of $T=293 \mathrm{~K}$. Thus, if hydrogen is supplied by diffusion from the bulk a hydrogen-assisted cleavage crack will propagate at a speed $v_{\mathrm{d}}^{\text {crit }} \leq 120 \mu \mathrm{m} \mathrm{s}^{-1}$ for $c_{\mathrm{L}} \leq 1 \mathrm{appm}$. Based on the results in Fig. $2 \mathrm{~b}$, such a slow growing cleavage crack will blunt by dislocation emission and arrest after a negligible amount of crack extension. We therefore conclude that for hydrogen-assisted cleavage to occur, a mechanism is needed to rapidly supply $\mathrm{H}$ to the crack-tip so as to maintain a cleavage crack velocity of at-least $5.4 \mathrm{~ms}^{-1}$. Diffusive flux from the lattice is unable to maintain such fast crack growth and this understanding shall form the basis of the new model proposed here.

\subsection{Overview of the hydrogen fracture of tensile bars}

As outlined in Section 1.1, the measured hydrogen-assisted toughness $J_{\mathrm{TH}}$ of high strength steels suggests that unrealistically large pre-existing flaws (cracks) are required to rationalise the measured tensile fracture strengths $\sigma_{\mathrm{f}}$. In a fracture toughness test for $J_{\mathrm{TH}}$ crack growth occurs in a controlled manner and thus typically is slow but the discussion above suggests that hydrogen-assisted cleavage fracture requires fast growing cracks. Here, we espouse a model to rationalise the observed fracture strengths (and a number of other characteristics of hydrogen induced fracture) by proposing that hydrogen induces fast-growing cracks, even under quasistatic remote loading. These fast-growing cracks not only limit crack-tip plasticity (such as crack-tip dislocation emission and twinning) but also reduce plasticity within the bulk in the vicinity of the crack-tip giving rise to a macroscopic quasi-cleavage failure mode. Critically, fracture of a quasi-statically loaded tensile bar by hydrogen-assisted fast-growing cracks implies that its tensile strength is decorrelated from the measured $J_{\mathrm{TH}}$ in an identical hydrogen environment.

We shall support the proposed model via detailed continuum finite element and atomistic calculations. However, it is instructive to first outline the entire mechanism before continuing to provide the appropriate details. The overall mechanism is illustrated in Fig. 3 and comprises two stages: a hydrogen-assisted crack growth stage-A and crack growth in the absence of hydrogen supply in stage-B. These two stages can be summarised as follows:

(i) Remote loading results in the formation and growth of small cavities. These cavities form either by the expansion of pre-existing flaws or more likely by debonding of inclusions such as carbides from the surrounding matrix ${ }^{1}$ (Fig. 3a). Hydrogen gas $\left(\mathrm{H}_{2}\right)$ fills these cavities via diffusive flux of $\mathrm{H}$ from the surrounding material (Fig. 3b). Under equilibrium conditions, the $\mathrm{H}_{2}$ gas pressure in the cavity equals the pressure $p_{\mathrm{H}}$ of the external hydrogen atmosphere.

(ii) The loading induces stress concentrations ahead of nanoscopic cracks/notches (associated with surface roughness) on the cavity surface. Cracks oriented along the (110) plane are very potent in initiating hydrogen-assisted cleavage due to the

\footnotetext{
${ }^{1}$ Throughout this manuscript, we shall refer to the entire debonded volume, which is partially filled with the carbide particle, as a void and the space within the void that is not occupied by the carbide as the cavity.
} 
formation of a hydride $\left(\mathrm{FeH}_{y}\right.$ where $\left.y \approx 1-3\right)$ over a nanometer size region at the crack-tips (Fig. 3c).

(iii) Hydrogen to form these hydrides is provided from the $\mathrm{H}_{2}$ gas in the cavity. This is because the large crack-tip stresses reduce the barriers for the entry of $\mathrm{H}$ from the cavity as well as for diffusion of $\mathrm{H}$ in a nanometer region around the crack-tip. This implies that the crack-tip hydrides can form rapidly allowing for a hydrogenassisted cleavage crack speed $v_{\mathrm{c}}>100 \mathrm{~ms}^{-1}$ (Fig. 3d). The key here is that this $\mathrm{H}$ supply route can sustain a cleavage velocity $v_{\mathrm{c}}>v_{\mathrm{blunt}}^{\text {crit }}$ and this significantly limits blunting via dislocation emission thereby allowing hydrogen-assisted cleavage to continue until the hydrogen supply within the cavity is exhausted. This is the end of stage-A.

(iv) Hydrogen-assisted fast crack growth induces a high strain-rate at the crack-tip which not only maintains a sharp crack-tip but also limits plasticity in the bulk around the crack-tip. This implies that given a sufficiently high remote applied stress $\sigma^{\infty}$, fast and brittle crack-growth can continue over large distances in stageB even after the hydrogen supply within the cavity is exhausted. It is this macroscopic fast crack growth that results in the final failure of the specimen (Fig. 3e)

Table 1: Summary of the material parameters for 4340 steel and $\mathrm{H}$.

\begin{tabular}{|l|l|l|}
\hline \multicolumn{1}{|c|}{ Material parameter } & Symbol & \multicolumn{1}{c|}{ Value } \\
\hline Young's modulus & $E$ & $200 \mathrm{GPa}$ \\
\hline Poisson's ratio & $v$ & 0.3 \\
\hline Yield strength & $\sigma_{\mathrm{Y}}$ & $1500 \mathrm{MPa}$ \\
\hline Strain hardening exponent & $n$ & 7.7 \\
\hline Reference strain-rate & $\dot{\varepsilon}_{0}$ & $0.1 \mathrm{~s}^{-1}$ \\
\hline Transition strain-rate & $\dot{\varepsilon}_{\mathrm{t}}$ & $10^{6} \mathrm{~s}^{-1}$ \\
\hline Rate sensitivity exponent & $m$ & 10 \\
\hline Solution enthalpy of H in 4340 steel & $\Delta H_{\mathrm{S}}$ & $27.1 \mathrm{~kJ} \mathrm{~mol}^{-1}$ \\
\hline Equilibrium constant in Sievert's law & $k$ & $1.08 \times 10^{3} \mathrm{appm} \mathrm{MPa}^{-1 / 2}$ \\
\hline Abel-Nobel equation of state constant for $\mathrm{H}_{2}$ & $B$ & $15.84 \times 10^{-6} \mathrm{~m}^{3} \mathrm{~mol}^{-1}$ \\
\hline Lattice constant of BCC Fe & $\mathcal{A}$ & $0.286 \mathrm{~nm}^{-1}$ \\
\hline Burgers vector magnitude & $b$ & $0.25 \mathrm{~nm}$ \\
\hline Pre-exponential diffusion factor & $D_{0}$ & $2.1 \times 10^{-7} \mathrm{~m}^{2} \mathrm{~s}^{-1}$ \\
\hline Lattice activation energy for H diffusion & $Q_{\mathrm{D}}$ & $6.6 \mathrm{~kJ} \mathrm{~mol} \mathrm{~mol}^{-1}$ \\
\hline Molar mass of Fe & $M$ & $56 \times 10^{-3} \mathrm{~kg} \mathrm{~mol}^{-1}$ \\
\hline Density of Fe & $\rho$ & $7850 \mathrm{~kg} \mathrm{~m}^{-3}$ \\
\hline Areal density of H binding sites on Fe surface & $\Gamma_{\mathrm{S}}$ & $2.65 \times 10^{-5} \mathrm{~mol} \mathrm{~m}^{-2}$ \\
\hline Hydrogen-assisted crack nucleation stress & $\Sigma_{\text {nuc }}$ & $3.6 \mathrm{GPa}$ \\
\hline Carbide particle radius & $R_{0}$ & $0.15 \mu \mathrm{m}$ \\
\hline Carbide particle number density & $N_{\mathrm{C}}$ & $5.5 \times 10^{11} \mathrm{~m}^{-3}$ \\
\hline Work of fracture for Fe & $G_{0}$ & $4.8 \mathrm{~J} \mathrm{~m}$ \\
\hline Fe cohesive strength & $T_{\mathrm{max}}$ & $15 \mathrm{GPa}$ \\
\hline Atomic vibration frequency of H & $\omega$ & $1014 \mathrm{~Hz}^{-2}$ \\
\hline & & \\
\hline
\end{tabular}



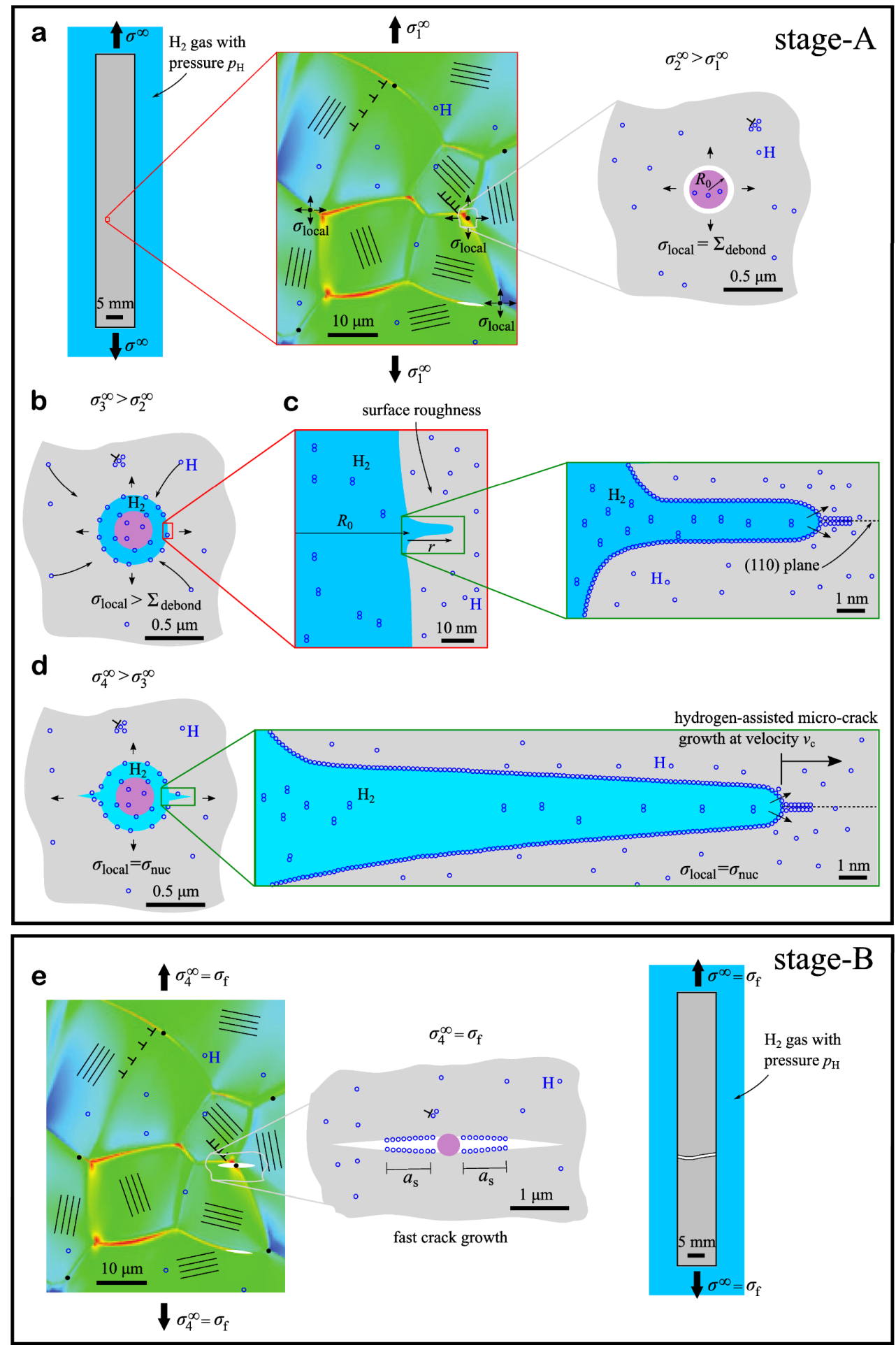

Figure 3: Summary of the overall mechanism for the tensile fracture of an alloy such as 4340 steel in a hydrogen environment. (a) Debonding of the metal matrix from an inclusion such as a carbide particle due to local stress concentrations. (b) Diffusive flow of hydrogen fills the cavity around the carbide. (c) Stress concentrations at nanoscopic crack-tips on the surface of the cavity drive the formation of a Fe hydride. (d) The Fe hydride promotes cleavage propagation of the crack with hydrogen supplied from within the cavity. This supply route rapidly replenishes the crack-tip hydride and drives the microcrack at a velocity $v_{c}>100 \mathrm{~ms}^{-1}$. (e) After the hydrogen within the cavity is exhausted this fast-growing microcrack continues to propagate, now in the absence of hydrogen, as the high crack-tip strain-rate suppresses plasticity. We refer to the hydrogen-assisted crack growth regime as stage-A with crack growth continuing in stage-B without a hydrogen supply. 


\section{A model for hydrogen induced fast-fracture}

We proceed now to describe the model based on the mechanism outlined above in three steps. First, in this section we present the model for a relatively high hydrogen pressure $p_{\mathrm{H}}$ (or equivalently high lattice hydrogen concentration $c_{\mathrm{L}}$ ) where fracture occurs at a remote stress $\sigma^{\infty}=\sigma_{\mathrm{f}}<\sigma_{\mathrm{Y}}$ such that there is no macroscopic plastic deformation of the specimen. In doing so we shall assume a priori that hydrogen promotes cleavage due to the formation of a cracktip hydride and that supply of hydrogen from within the cavity results in a fast-growing cleavage crack until hydrogen supply within the cavity is exhausted. Using this a priori assumption we present continuum simulations and predictions for the macroscopic tensile fracture of the specimen. Then in Section 4 we present detailed atomistic calculations to justify these a priori assumptions, viz. the formation of a hydride at the crack-tip, cleavage in the presence of the hydride and the rate at which the hydride is expected to be replenished via hydrogen supplied from within the cavity. Finally, in Section 5 we extend the model to lower levels of $p_{\mathrm{H}}$ when fracture is accompanied by macroscopic plasticity such that $\sigma_{\mathrm{f}} \geq \sigma_{\mathrm{Y}}$.

In developing the model, we shall make numerous simplifying assumptions with regards the micro-structure of the steel as well as the various mechanical processes. We emphasize that these assumptions are not expected to change the qualitative (and in some cases even quantitative) features of the predictions. In fact, in most cases these assumptions can be relatively easily relaxed if the microstructure of the steel were known precisely so as to permit a detailed calculation of the fracture process in a given specimen.

\subsection{Description of the overall problem of the tensile loading of a bar in a hydrogen environment}

We present a mechanism for the tensile fracture of a smooth steel bar loaded in a hydrogen environment. To aid the discussion we shall think of a gaseous hydrogen environment maintained at a hydrogen partial pressure $p_{\mathrm{H}}$ although the analysis remains valid for other charging environments as well. The tensile loading rate $\dot{\varepsilon}^{\infty}$ is assumed to be sufficiently slow such that hydrogen within the specimen is in equilibrium with the external hydrogen atmosphere up to the onset of fracture, i.e. the chemical potential of the atomic hydrogen within the specimen is equal to that of the hydrogen gas in the surrounding atmosphere. In the absence of hydrogen, the uniaxial tensile stress versus strain response of the 4340 steel is adequately described by an elastic-plastic law with a strain hardening response

$$
\bar{\sigma}=\sigma_{\mathrm{Y}}\left(1+E \frac{\varepsilon^{\mathrm{p}}}{\sigma_{\mathrm{Y}}}\right)^{1 / n},
$$

where $\bar{\sigma}$ is the uniaxial tensile flow strength at a tensile plastic strain $\varepsilon^{\mathrm{p}}$. Here $E$ is the Young's modulus, $n$ the hardening exponent and $\sigma_{\mathrm{Y}}$ the yield strength. Then, in the absence of hydrogen embrittlement the tensile ductility of the steel follows from the Considère criterion as $1 / n-$ $\sigma_{\mathrm{Y}} / E$ with the failure strength, normally referred to as the ultimate tensile strength $\sigma^{\mathrm{UTS}}$, given by substituting $\varepsilon^{\mathrm{p}}=1 / n-\sigma_{\mathrm{Y}} / E \approx 1 / n$ in Eq. (3.1). The constitutive parameters for 4340 steel including the elastic Poisson's ratio $v$ are listed in Table 1.

Novak et al. (2010) reported an extensive microstructural characterisation of the quenched and tempered 4340 steel they investigated. In brief, the steel comprised grains with an average size $\sim 10 \mu \mathrm{m}$ along with carbide particles (distribution with modal and maximum sizes of $0.3 \mu \mathrm{m}$ and $2 \mu \mathrm{m}$, respectively) mainly at the grain boundaries and $\mathrm{MnS}$ particles of sizes in the range $1-10 \mu \mathrm{m}$ distributed throughout the matrix. A detailed characterization of hydrogen traps within this steel was also reported and the authors concluded that three primary trap types were operative: (i) dislocation traps with a binding enthalpy $\Delta H=-18 \mathrm{~kJ} \mathrm{~mol}^{-1}$; (ii) grain 
boundary traps with a binding enthalpy $\Delta H=-48 \mathrm{~kJ} \mathrm{~mol}^{-1}$ and (iii) carbide traps with a binding enthalpy $\Delta H=-72 \mathrm{~kJ} \mathrm{~mol}^{-1}$. Under equilibrium conditions, the occupancies of these traps can be related to the lattice hydrogen concentration $c_{\mathrm{L}}$ via the Oriani (1970) model; readers are referred to Sofronis and McMeeking (1989) for details but here it suffices to say that at room temperature for $c_{\mathrm{L}}>0.1$ appm both the carbide and grain boundary traps are fully occupied while for most realistic levels of $c_{\mathrm{L}}$ the dislocation traps remain relatively empty. However, as discussed in Section 1.2 the occupancy of traps does not correlate with the fracture strength and in fact we shall not explicitly require knowledge of the occupancy of these traps in the model developed here. Nevertheless, it is worthwhile keeping these trap enthalpies in mind as they will serve as a useful reference to compare with the atomistically computed binding energies (Section 4) of hydrogen sites that form around the loaded crack tip.

The lattice hydrogen concentration $c_{\mathrm{L}}$ in a bar subjected to uniaxial tension under equilibrium conditions is nominally spatially uniform and directly relates to the pressure $p_{\mathrm{H}}$ of gaseous hydrogen in the external atmosphere. With $c_{\mathrm{L}}$ in units of appm, this relation is given by Sieverts law in terms of an equilibrium constant $k=1.08 \times 10^{3} \mathrm{appm} \mathrm{MPa}^{-1 / 2}$ as (Nelson and Stein, 1973)

$$
c_{\mathrm{L}}=k \exp \left(-\frac{\Delta H_{\mathrm{s}}}{R T}\right) \sqrt{f},
$$

where $\Delta H_{\mathrm{s}}=27.1 \mathrm{~kJ} \mathrm{~mol}^{-1}$ is the solution enthalpy of the hydrogen in 4340 steel and $f$ the fugacity of the hydrogen gas in $\mathrm{MPa}$. To relate the fugacity to $p_{\mathrm{H}}$, we require an equation of state for hydrogen gas. If hydrogen is assumed to be an ideal gas $f=p_{\mathrm{H}}$ but it is well-known that hydrogen under normal charging pressures displays non-ideal behaviour with the AbelNoble equation of state (San Marchi et al., 2007) a more appropriate description. The AbelNoble equation relates the molar density $\rho_{\mathrm{H}}$ of $\mathrm{H}_{2}$ to $p_{\mathrm{H}}$ as

$$
\rho_{\mathrm{H}}=\frac{p_{\mathrm{H}}}{R T+B p_{\mathrm{H}}},
$$

with $B=15.84 \times 10^{-6} \mathrm{~m}^{3} \mathrm{~mol}^{-1}$. The fugacity then follows from a calculation of the chemical potential of $\mathrm{H}_{2}$ using this equation of state and is given by

$$
f=p_{\mathrm{H}} \exp \left(\frac{p_{\mathrm{H}} B}{R T}\right) \text {. }
$$

Novak et al. (2010) reported data for the fracture strength of 4340 for $c_{\mathrm{L}}$ in the range 0 appm to 1.0 appm which then corresponds to hydrogen gas charging pressures $0 \mathrm{MPa} \leq p_{\mathrm{H}} \leq$ $366 \mathrm{MPa}$. We shall now proceed to develop the model for a relatively high hydrogen pressure $p_{\mathrm{H}}$ (or equivalently high lattice hydrogen concentration $c_{\mathrm{L}}$ ) where fracture occurs at a remote stress $\sigma^{\infty}=\sigma_{\mathrm{f}}<\sigma_{\mathrm{Y}}$ such that there is no macroscopic plastic deformation of the specimen.

\subsection{Debonding of inclusions and filling of cavities with hydrogen}

Flaws in terms of pre-existing cracks and voids are typically absent in high strength engineering steels. Rather voids are nucleated during loading in such materials by debonding of the matrix from inclusions such as carbides and MnS (McClintock, 1968). Thus, here we restrict ourselves to cavities formed by such a debonding process although of course the idea can readily be generalised to other mechanisms for the formation of cavities. For simplicity we assume the inclusions of interest to be spheres of radius $R_{0}$ that are perfectly bonded to the surrounding material. Typically, there exists a distribution of inclusion sizes but here we shall not present a statistical analysis. Rather, because the model requires hydrogen-assisted crack growth to be triggered by a local stress concentration, it is imperative that an inclusion is present at such a 
location. Thus, we identify $R_{0}$ with the inclusion size corresponding to the mode of the distribution of inclusion sizes.

Inclusions such as carbides debond from the surrounding material when the local hydrostatic stress at the inclusion reaches a critical value $\Sigma_{\text {debond. The literature on the }}$ measurement/prediction of the stress to induce debonding and nucleate voids is sparse although a combined experimental and numerical investigation (Giovanola et al., 2006) has suggested that $\Sigma_{\text {debond }} \approx 3 \mathrm{GPa}$ in 4340 steels. Debonding of the inclusions is known to occur early in the loading history (typically when the remote stress $\sigma^{\infty} \ll \sigma_{\mathrm{Y}}$ ) and it thus follows that debonding is driven by local stress concentrations (e.g. grain boundary triple junctions); see Fig. 3a. In fact, at a fixed remote stress $\sigma^{\infty}$ there exists a wide distribution of local stresses $\sigma_{\text {local }}$ around inclusions that drive the debonding process with $\sigma_{\text {local }}$ for a specific inclusion relatively de-correlated from $\sigma^{\infty}$. Given the constraint imposed by the elastic material surrounding the inclusion, we assume that the field due to the stress concentration is wellapproximated by a plane strain field such that the principal stresses around the inclusion are $\left(\sigma_{\text {local }}, \sigma_{\text {local }}, v \sigma_{\text {local }}\right)$ and a void nucleates around this inclusion when $\sigma_{\text {local }} \approx \Sigma_{\text {debond }}$ (since we are not explicitly modelling the micro-structure we shall not calculate $\sigma_{\text {local }}$ but rather assume there exist locations within the specimen where $\sigma_{\text {local }}$ attain the value $\Sigma_{\text {debond }}$ required to cause debonding). Continued remote loading increases $\sigma_{\text {local }}$ beyond this critical value and an ellipsoidal void grows around the inclusion associated with principal strains $\left(\varepsilon_{\text {local }}, \varepsilon_{\text {local }}, 0\right)$. Neglecting any effect of the debonded inclusion, the volume of the cavity around the inclusion is related to $\sigma_{\text {local }}$ via

$$
\Delta V=\frac{4 \pi}{3} R_{0}^{3}\left[\left(1+\varepsilon_{\text {local }}\right)^{2}-1\right]
$$

where $\varepsilon_{\text {local }}=\sigma_{\text {local }}\left(1-v-2 v^{2}\right) / E$. Here we have assumed that the stress field $\sigma_{\text {local }}$ is spatially uniform over a length scale on the order of the inclusion size $R_{0}$ and that local deformation is primarily elastic. We shall consider the case of plastic deformations around the inclusions in Section 5.

Debonding of the inclusion induces a diffusive flux of atomic hydrogen from within the bulk of the specimen towards the cavity so as to both cover the newly formed Fe surface with $\mathrm{H}$ and fill the cavity with hydrogen gas. This tendency to fill the cavity and cover the surface is a result of the fact that the enthalpy of hydrogen gas with respect to $\mathrm{H}$ in bulk Fe is $-\Delta H_{\mathrm{s}}=$ $-27 \mathrm{~kJ} \mathrm{~mol}^{-1}$ (Nelson and Stein, 1973; Sorescu, 2005) while atomic hydrogen on Fe surfaces has a binding enthalpy $\Delta H \approx-96 \mathrm{~kJ} \mathrm{~mol}^{-1}$ (Jiang and Carter, 2004a; Zou et al., 2012). Recall that we are considering the limit of slow loading of the specimen such that prior to the onset of fracture, equilibrium conditions are always maintained. Thus, under equilibrium conditions, for any realistic value of $c_{\mathrm{L}}$ (i.e. $c_{\mathrm{L}} \geq 0.01 \mathrm{appm}$ ), we anticipate all $\mathrm{H}$ sites on the cavity surface to be fully occupied while hydrogen gas will be at a pressure $p_{\mathrm{H}}$ within the cavity, i.e. under equilibrium conditions the hydrogen gas pressure within the cavity will equal that of the external hydrogen atmosphere with $c_{\mathrm{L}}$ in the specimen given by Eqs. (3.2) and (3.4). Further, we also assume that the hydrogen pressure within the cavity is sufficiently small so that filling of the cavity with hydrogen does not significantly affect the cavity volume estimate given by Eq. (3.5).

\subsection{Fast microcrack growth driven by hydrogen supplied from within the cavity}

Detailed atomistic calculations presented in Section 4 demonstrate that (i) mode-I loading of cracks allows for a hydride to form in about a $1.25 \mathrm{~nm}$ region ahead of the crack-tip and (ii) this hydride induces cleavage at a critical energy release rate $G_{\mathrm{I}} \approx 4.2 \mathrm{~J} \mathrm{~m}^{-2}$. (for the 
crystallographic orientation considered in Section $4, G_{\mathrm{I}} \approx 4.2 \mathrm{~J} \mathrm{~m}^{-2}$ corresponds to $K_{\mathrm{I}}=$ 1.0 MPa $\sqrt{\mathrm{m}}$; see Appendix B). Moreover, with hydrogen being supplied from within the cavity, the crack-tip hydride is replenished rapidly resulting in cleavage crack propagation of a microcrack emanating from the cavity at a velocity $v_{\mathrm{c}} \approx 100 \mathrm{~ms}^{-1}$. Here, we use these findings to estimate (a) the stress $\sigma_{\text {local }}$ required to induce cleavage from cracks associated with the roughness of the cavity surface and (b) the extent to which these cracks can propagate by hydrogen-assisted cleavage.

We model the nanoscopic cracks associated with the cavity surface roughness as penny-shaped cracks that emanate a distance $r$ from the surface of a spherical void of radius $R_{0}$ and loaded by a remote hydrostatic stress $\sigma_{\text {local }}$. With deformations constrained to remain elastic, the surface of the void is then loaded by a tensile stress $\sigma=K_{\mathrm{T}} \sigma_{\text {local }}$ where $K_{\mathrm{T}}=2$ is the stress concentration factor for a spherical void in an infinite medium subjected to remote hydrostatic loading $\sigma_{\text {local }}$. Then in the limit of a small surface crack $r \ll R_{0}$, the stress-intensity factor $K_{\mathrm{I}}$ is well-approximated by the stress intensity factor of a penny-shaped crack of radius $r$ subjected to a remote stress $\sigma$ (Francois et al., 2012) and $K_{\mathrm{I}}$ follows as

$$
K_{\mathrm{I}}=\frac{4}{\pi} \sigma_{\text {local }} \sqrt{\pi r}
$$

The atomistic calculations of Section 4 suggest hydrogen-assisted cleavage crack growth occurs at $K_{\mathrm{I}}=1.0 \mathrm{MPa} \sqrt{\mathrm{m}}$ in BCC Fe for a crack with crack plane (110) and crack front direction [110]. Setting this value for $K_{\mathrm{I}}$ in Eq. (3.6) we observe that cleavage will initiate when $\sigma_{\text {local }}=\Sigma_{\text {nuc }} \approx 14 / \sqrt{r} \mathrm{GPa}$, where $r$ is in units of nanometer. Thus, the local stress required to initiate cleavage is strongly dependent on the precise cavity surface roughness. Nevertheless, we can make an assessment on what types of surface cracks will tend to propagate by hydrogen-assisted cleavage.

In the presence of hydrogen, the above analysis suggests that a crack of size $r>22 \mathrm{~nm}$ will cleave at $\Sigma_{\text {nuc }}<3 \mathrm{GPa}$. However, recall that the cavity forms by debonding of the inclusion from the matrix at $\sigma_{\text {local }}=\Sigma_{\text {debond }}=3 \mathrm{GPa}$ and at the point of formation of the cavity no hydrogen is present within the cavity. Thus, immediately upon formation of the cavity there is no hydrogen supply to rapidly form the crack-tip hydride. In the absence of hydrogen, cracktip dislocation emission also occurs at $K_{\mathrm{I}}=K_{\text {emit }}=1.0 \mathrm{MPa} \sqrt{\mathrm{m}}$ and thus we anticipate that cracks of size $r>22 \mathrm{~nm}$ will blunt and remain dormant. Remarkably, it is the shorter cracks that will tend to propagate via the hydrogen-assisted cleavage mechanism as these cracks will be loaded to $K_{\mathrm{I}}=1.0 \mathrm{MPa} \sqrt{\mathrm{m}}$ after loading is continued beyond the debonding event, giving sufficient time for hydrogen gas to fill the cavity. This thus sets a lower limit on $\sigma_{\text {nuc }}$, i.e. $\Sigma_{\text {nuc }}>3 \mathrm{GPa}$. Here we shall present the bulk of our simulations for an assumed cleavage stress $\Sigma_{\text {nuc }}=3.6 \mathrm{GPa}$ but shall also show that the sensitivity of the predictions to the precise value of $\sigma_{\text {nuc }}$ is mild over the range $3 \mathrm{GPa} \leq \Sigma_{\text {nuc }} \leq 4.5 \mathrm{GPa}$. Thus, while $\Sigma_{\text {debond }}$ and $\Sigma_{\text {nuc }}$ are material parameters we only explicitly need information on $\Sigma_{\text {nuc }}$ for predicting the onset of hydrogen-assisted cleavage from the surface of the cavity and the reference value of $\Sigma_{\text {nuc }}$ is listed in Table 1.

Given the appropriate conditions, as parameterised here through the value of the local stress $\sigma_{\text {local }}=\Sigma_{\text {nuc }}$, hydrogen-assisted cleavage initiates from the surface of the cavity. We now proceed to estimate the distance this crack will propagate fuelled by hydrogen supplied from within the cavity. At the instant that cleavage commences, the volume of the cavity is given by Eq. (3.5) with $\varepsilon_{\text {local }}$ replaced by $\varepsilon_{\text {nuc }}=\Sigma_{\text {nuc }}\left(1-v-2 v^{2}\right) / E$. We label this volume as $\Delta V_{\text {nuc }}$. Cleavage growth of the microcrack consumes hydrogen with $\mathrm{H}$ occupying all surface sites on 
the newly created surfaces. With hydrogen supply restricted to that available as gaseous hydrogen in the cavity, mass balance provides a relation between the crack extension $a_{\mathrm{s}}$ and the molar density $\rho_{\mathrm{H}}$ of hydrogen gas within the cavity prior to the onset of crack extension as

$$
2 \Gamma_{\mathrm{s}} \pi\left[\left(R_{0}+a_{\mathrm{s}}\right)^{2}-R_{0}^{2}\right]=2 \Delta V_{\text {nuc }} \rho_{\mathrm{H}} .
$$

Here we have included a factor of two on the left-hand side to emphasize that atomic decohesion results in the formation of two crack surfaces while the factor of two on the righthand side indicates that the $\mathrm{H}_{2}$ gas in the cavity dissociates into two $\mathrm{H}$ atoms. We emphasize that crack extension with $\mathrm{H}$ being supplied from within the cavity occurs at a speed $v_{\mathrm{c}} \approx$ $100 \mathrm{~ms}^{-1}$ and there is negligible supply of $\mathrm{H}$ via diffusive flux from within the specimen bulk over the duration $a_{\mathrm{s}} / v_{\mathrm{c}}$ of crack growth in this mode. An expression for the crack extension $a_{\mathrm{s}}$ in terms of the hydrogen gas pressure $p_{\mathrm{H}}$ (recall that under equilibrium conditions $p_{\mathrm{H}}$ in the cavity prior to crack growth equals the pressure of the external hydrogen atmosphere) then follows from the equation of state (3.3) as

$$
a_{\mathrm{s}}=\sqrt{\frac{p_{\mathrm{H}} \Delta V_{\text {nuc }}}{\Gamma_{\mathrm{s}} \pi\left(R T+B p_{\mathrm{H}}\right)}+R_{0}^{2}}-R_{0} .
$$

Further cleavage crack growth via this hydrogen-assisted mechanism is precluded as the hydrogen supply within the cavity is exhausted.

\subsection{Continued brittle fracture in the absence of hydrogen (stage-B)}

The hydrogen gas within the cavity is consumed when the microcracks attain a radius $R_{0}+a_{\mathrm{s}}$ via a hydrogen-assisted fast cleavage mode. In this section we investigate conditions required for these fast-growing microcracks to continue to grow even in the absence of a hydrogen supply and thereby result in macroscopic fracture.

Following the discussion of Section 2 it is clear that blunting by dislocation emission is kept minimal for cracks growing at $v_{\mathrm{c}} \approx 100 \mathrm{~ms}^{-1}$. However, macroscopic crack growth will inevitably result in the crack triggering plasticity in the bulk as the crack front crosses dislocation sources such as Frank-Read sources and grain boundaries. The bulk plasticity triggered by this macroscopic crack growth usually implies that cleavage fracture is precluded in metals such as 4340 steel in the absence of hydrogen. There is however an exception as first analysed by Freund and Hutchinson (1985): fast-growing cracks limit plasticity around the crack-tip such that high velocity cracks can grow at $K_{\mathrm{I}} \ll K_{\mathrm{IC}}$, where $K_{\mathrm{IC}}$ is the fracture toughness of the material in the limit of slow crack growth. This framework was later extended by Landis et al. (2000) to investigate the steady-state toughness of rate dependent materials using a cohesive zone to model the fracture process. Here we shall use a similar analysis to investigate the continued growth of the microcrack but now in the absence of hydrogen. With crack-tip dislocation emission inhibited for the fast-growing crack it suffices to model the cleavage fracture process via a cohesive zone framework with plasticity in the bulk modelled using a rate dependent continuum plasticity model for the polycrystalline steel.

Consider the axi-symmetric boundary value problem sketched in Fig. 4. It comprises of a large domain of radius $W$ and height $H=10 \mathrm{~W}$ with a centrally located spherical cavity of radius $R_{0}$ subjected to a remote tensile stress $\sigma^{\infty}$. Recalling that $R_{0}$ is set by the size of carbide particles, the domain size $W \gg R_{0}$ and in the calculations presented here we set $W / R_{0}=10$ : further increasing $W / R_{0}$ had no effect on the numerical results presented here. Crack growth is modelled to occur on a diametrical plane of the cavity perpendicular to the direction of loading. The bulk material is assumed to be a $J_{2}$ flow theory elastic-plastic solid with a strain 
hardening response in the limit of a vanishing plastic strain-rate $\dot{\varepsilon}^{\mathrm{p}}$ given by Eq. (3.1). The rate sensitivity of $\bar{\sigma}$ is accounted for by modifying Eq. (3.1) to

$$
\bar{\sigma}=\sigma_{0}\left(1+E \frac{\varepsilon^{\mathrm{p}}}{\sigma_{0}}\right)^{1 / n},
$$

with the rate dependent strength $\sigma_{0}$ given by

$$
\sigma_{0}= \begin{cases}\sigma_{\mathrm{Y}}\left(1+\frac{\dot{\varepsilon}^{\mathrm{p}}}{\dot{\varepsilon}_{0}}\right)^{\frac{1}{m}}, & \dot{\varepsilon}^{p}<\dot{\varepsilon}_{\mathrm{t}} \\ \sigma_{\mathrm{Y}}\left(1+\frac{\dot{\varepsilon}_{\mathrm{t}}}{\dot{\varepsilon}_{0}}\right)^{\frac{1}{m}} \frac{\dot{\varepsilon}^{\mathrm{p}}}{\dot{\varepsilon}_{\mathrm{t}}}, & \dot{\varepsilon}^{\mathrm{p}} \geq \dot{\varepsilon}_{\mathrm{t}} .\end{cases}
$$

Here $\dot{\varepsilon}_{0}$ is a reference strain-rate with $m$ the rate sensitivity exponent and $\dot{\varepsilon}_{\mathrm{t}}$ is a transition strain-rate at which the rate sensitivity switches from a power-law dependence on $\dot{\varepsilon}^{\mathrm{p}}$ to a linear dependence set by the drag on the motion of dislocations due to phonon scattering. Representative values of the rate dependency parameters for 4340 steel are: $\dot{\varepsilon}_{0}=0.1 \mathrm{~s}^{-1}, \dot{\varepsilon}_{\mathrm{t}}=$ $10^{6} \mathrm{~s}^{-1}$ and $m=10$ (Armstrong et al., 2009).
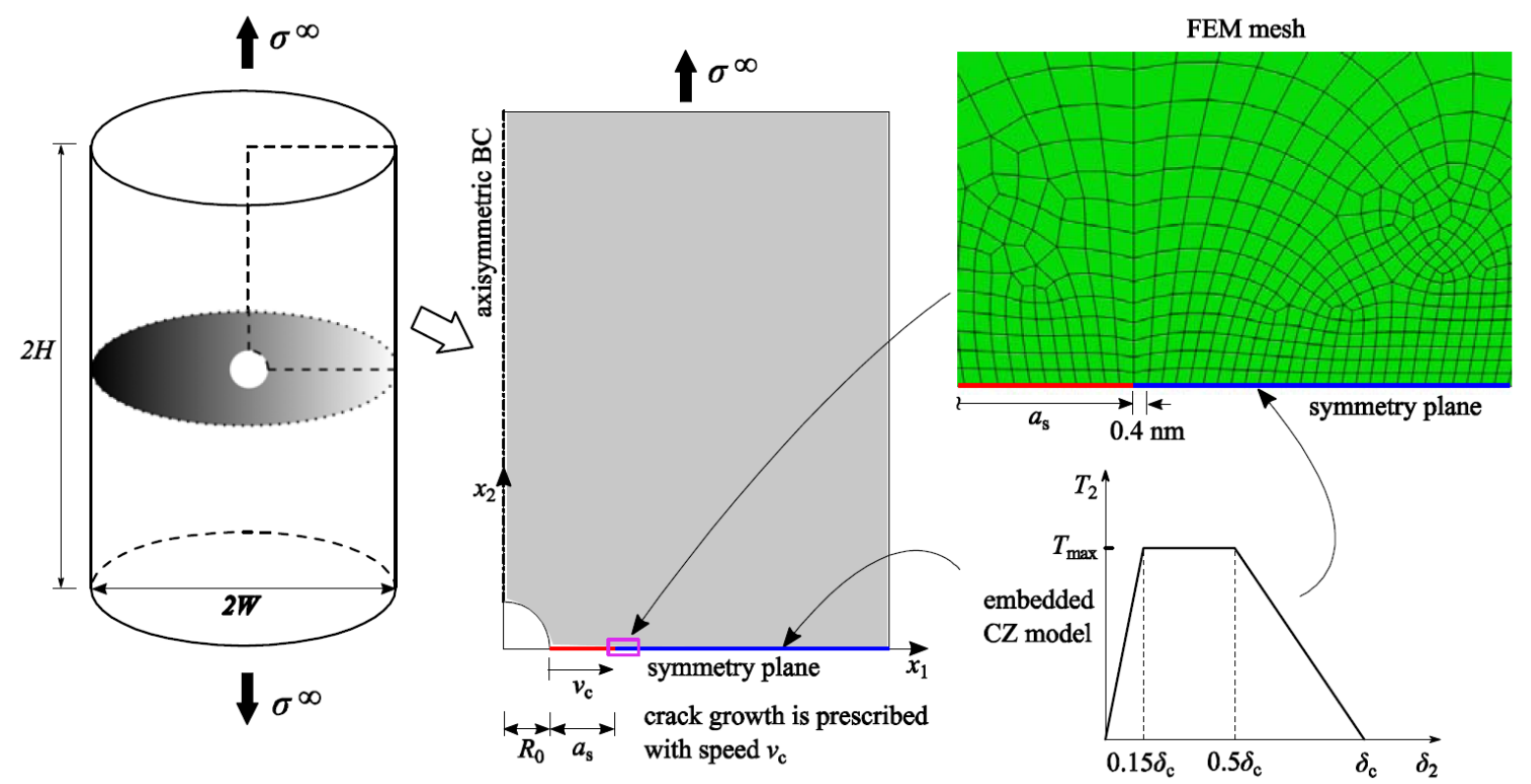

Figure 4: Sketch of the FE boundary value for modelling continued propagation of the microcrack but now in the absence hydrogen supply. In the FE model a micro-crack of length $a_{\mathrm{s}}$ is first propagated from the cavity surface of radius $R_{0}$ at a speed $v_{\mathrm{c}}$ and its subsequent propagation for a fixed remote tensile stress $\sigma^{\infty}$ investigated as an outcome of the FE solution. The crack propagation modelled via a cohesive surface framework along with rate-dependent plasticity in the bulk.

The basic numerical formulation is standard and here we provide some salient details so as to aid the discussion of the results that follow. Symmetry of the problem implies that it is sufficient to model a quarter of the domain as shown in Fig. 4 with vanishing shear tractions along the plane of crack growth. Cleavage is modelled via cohesive zone framework wherein the crack opening traction $T_{2}$ is prescribed in terms of the separation $\delta_{2}=2 u_{2}$ for material points lying on the $x_{2}=0$ plane where $u_{2}$ is the displacement of material points on the crack plane in the $x_{2}$-direction; see Fig. 4 . We take this $T_{2}-\delta_{2}$ relation to be of the form employed by Tvergaard and Hutchinson (1992) such that the maximum opening traction $T_{\max }$ and the critical separation $\delta_{\mathrm{c}}$ for the formation of the crack are related to the work of separation via $G_{0} \equiv 0.675 T_{\max } \delta_{\mathrm{c}}$; see Fig. 4 . Then with the work of separation $G_{0}=4.8 \mathrm{~J} \mathrm{~m}^{-2}$ for Fe in the 
absence of $\mathrm{H}$ (Jiang and Carter, 2004b) along with the choice $\delta_{\mathrm{c}} \approx 1.6 \mathcal{A}$, where $\mathcal{A}=$ $0.286 \mathrm{~nm}$ is the lattice parameter of $\mathrm{Fe}$, it follows that $T_{\max } \approx 15 \mathrm{GPa}$. Our aim is to investigate the continued growth of the microcracks after the supply of hydrogen from the cavity is exhausted and we do this by considering the following boundary/initial value problem. The specimen with the spherical void of radius $R_{0}$ is subjected to slow remote tensile straining until a specified remote stress $\sigma^{\infty}$ is attained and then this remote stress is held fixed. This is the remote stress at which we assume hydrogen-assisted microcrack growth occurs and we wish to determine whether this microcrack growth results in macroscopic fracture at this specified value of $\sigma^{\infty}$. Hydrogen-assisted microcrack growth is then specified by growing a crack from the cavity surface along $x_{2}=0$ plane in the positive $x_{1}$-direction at a speed $v_{\mathrm{c}}$ to a distance $a_{\mathrm{s}}$ from the cavity surface. No further crack growth is prescribed with subsequent temporal evolution of crack growth (i.e. $\Delta a$ ) obtained as an outcome of the solution of this boundary/initial value problem. The calculations were conducted using an explicit dynamic (material density $\rho=7850 \mathrm{~kg} \mathrm{~m}^{-3}$ ) finite element (FE) method in a finite strain setting with a mesh comprising 4-noded axisymmetric elements as shown in Fig. 4 (a mesh size of about $0.4 \mathrm{~nm}$ was employed around the crack-tip to resolve the large gradients associated with the cohesive zone model). The main aim of the calculations was to determine the minimum stress $\sigma^{\infty}=\sigma_{\mathrm{f}}$ at which runaway crack growth occurs: this value of $\sigma_{\mathrm{f}}$ is then defined as the tensile failure strength of the specimen.

For the specified material properties of 4340 steel (Table 1), we use dimensional analysis to write $\sigma_{\mathrm{f}}$ in terms of the hydrogen-dependent loading parameters viz. $a_{\mathrm{s}}$ and $v_{\mathrm{c}}$, i.e.

where

$$
\bar{\sigma}_{f} \equiv \frac{\sigma_{\mathrm{f}}}{\sigma_{\mathrm{Y}}}=\mathcal{F}\left[\frac{a_{\mathrm{s}}}{R_{0}}, \frac{v_{\mathrm{c}}}{r_{\mathrm{p}} \dot{\varepsilon}_{\mathrm{t}}}\right] \text {, }
$$

$$
r_{\mathrm{p}}=\frac{1}{3 \pi}\left(\frac{E}{1-v^{2}}\right) \frac{G_{0}}{\sigma_{\mathrm{Y}}^{2}}
$$

is a measure of the plane strain plastic zone size at an applied mode-I energy release rate $G_{\mathrm{I}}=$ $G_{0}$ and $\bar{v}_{\mathrm{c}} \equiv v_{\mathrm{c}} /\left(r_{\mathrm{p}} \dot{\varepsilon}_{\mathrm{t}}\right)$ is a measure of ratio of the strain-rate at the crack-tip to the transition strain-rate $\dot{\varepsilon}_{\mathrm{t}}$. Recall that $G_{0}$ is not the quasi-static toughness $G_{\text {IC }}$ of 4340 steel in the absence of hydrogen but rather just the work of separation of Fe in the absence of hydrogen. In fact, under quasi-static conditions a cohesive zone model with $T_{\max } / \sigma_{\mathrm{Y}}=10$ would predict $G_{\mathrm{IC}} \rightarrow$ $\infty$ (i.e. $\gg G_{0}$ ) as plastic deformation of the metal would prevent the crack opening stresses to build-up to $T_{\max }=15 \mathrm{GPa}$ required for decohesion; see Tvergaard and Hutchinson (1992). We shall now present numerical results for the functional dependence of $\sigma_{\mathrm{f}}$ as specified in Eq. (3.11). All results pertain to an inclusion of radius $R_{0}=0.15 \mu \mathrm{m}$ that corresponds to the mode of distribution of carbide sizes in the 4340 steel investigated by Novak et al. (2010).

With the crack-tip defined as the location where the opening $\delta_{2}=\delta_{\mathrm{c}}$, predictions of the evolution of the normalized crack growth crack extension $\Delta a / a_{\mathrm{s}}$ as a function of normalized time are included in Fig. 5a. Here $\bar{t} \equiv t \dot{\varepsilon}_{\mathrm{t}}$ with time $t=0$ corresponding to the instant when the crack had emanated a distance $a_{\mathrm{s}}$ from the cavity surface via hydrogen-assisted cleavage (i.e. prescribed in the FE calculation) and $\Delta a$ is the further crack extension that is an outcome of the FE solution. The results in Fig. 5a are for the choice $a_{\mathrm{s}}=0.25 \mu \mathrm{m}$, a hydrogen-assisted microcrack speed $v_{\mathrm{c}}=100 \mathrm{~ms}^{-1}\left(\bar{v}_{\mathrm{c}} \approx 2000\right)$ and a range of remote tensile stresses $\sigma^{\infty}$. In all cases, $\Delta a>0$, i.e. after the hydrogen from the cavity is exhausted, the crack continues to grow via a cleavage mechanism. However, at low values of $\sigma^{\infty}$ this crack growth is minimal with the crack arresting and consequently not resulting in overall fracture of the specimen. 
However, for applied stresses $\sigma^{\infty}>\sigma_{\mathrm{f}}=830 \mathrm{MPa}$ runaway crack growth ensues resulting in specimen fracture.
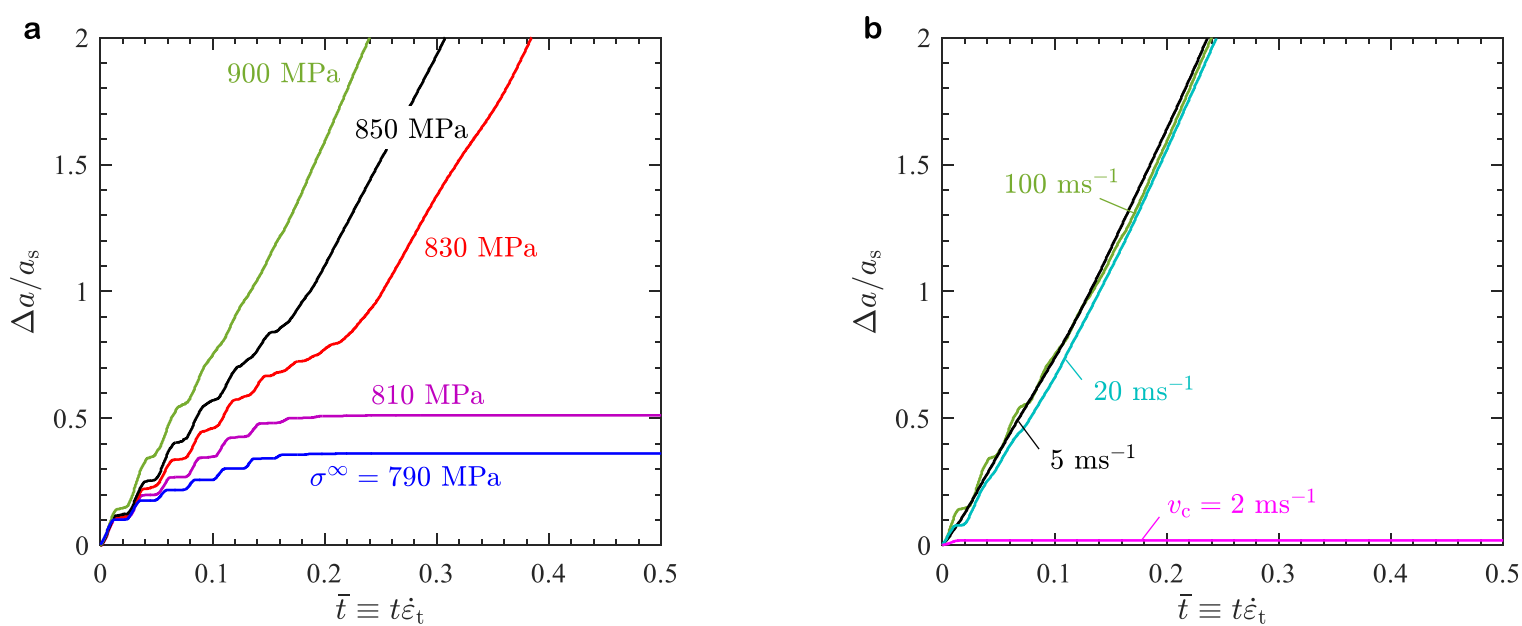

Figure 5: (a) FE predictions of the normalised crack extension $\Delta a / a_{\mathrm{s}}$ as a function of normalised time $\bar{t} \equiv t \dot{\varepsilon}_{\mathrm{t}}$ for the case of a hydrogen-assisted microcrack velocity $v_{\mathrm{c}}=100 \mathrm{~ms}^{-1}$ and selected levels of remote applied stress $\sigma^{\infty}$. (b) The corresponding predictions for a fixed applied stress $\sigma^{\infty}=900 \mathrm{MPa}$ and selected values of $v_{\mathrm{c}}$. All results are for a hydrogen-assisted microcrack of length $a_{\mathrm{s}}=0.25 \mu \mathrm{m}$ and a cavity of radius $R_{0}=0.15 \mu \mathrm{m}$. Time $t=0$ corresponds to the instant when crack growth is no longer prescribed and the crack has emanated a length $a_{\mathrm{s}}$ from the cavity surface by hydrogen-assisted growth.

It is worth emphasizing here that fracture at $\sigma_{\mathrm{f}}=830 \mathrm{MPa}$ with $a_{\mathrm{s}}=0.25 \mu \mathrm{m}$ would be precluded in the absence of the initial fast-growing hydrogen-assisted crack. To understand this, recall that with $T_{\max } / \sigma_{\mathrm{Y}}=10$, the quasi-static analysis of Tvergaard and Hutchinson (1992) predicts a steady-state fracture toughness $K_{\mathrm{ss}} \rightarrow \infty$ as plastic flow of the material around the crack-tip prevents the crack opening stress from attaining the value $T_{\max }$ required to initiate crack growth. Thus, for the parameters employed here runaway crack growth would have been precluded for any finite $\sigma_{\mathrm{f}}$ by that analysis. In our analysis specimen fracture occurs due to the high strain-rates induced at the crack-tip by the initial crack speed $v_{c}$. Material strain-rate sensitivity then increases the yield strength and allows the material to sustain crack opening stresses $\geq T_{\max }$ thereby permitting crack growth and fracture to occur with minimal plastic deformation and crack-tip blunting. We illustrate this in Fig. 6 where we include spatial distributions of the von-Mises effective plastic strain $\varepsilon_{\mathrm{e}}^{\mathrm{p}}$ in a region around the crack-tip at selected values of the normalized time $\bar{t}$ for an applied stress $\sigma^{\infty}=900 \mathrm{MPa}$. Clearly plasticity is confined to only in the immediate vicinity of the crack (for the case in Fig. 6 to a $0.2 \mu \mathrm{m}$ region) and thus this fracture can be classified as quasi-cleavage, i.e. brittle fracture with very limited plasticity - such fracture surfaces are typically observed for the tensile fracture of quasistatically loaded steels in a hydrogen environment (Martin et al., 2019).

The quasi-cleavage fracture predicted here is predicated on the fact that at $\sigma^{\infty}>\sigma_{\mathrm{f}}$, a fastgrowing cleavage crack has been initiated by feeding of hydrogen from the internal cavity around an inclusion. To illustrate this effect, we include, in Fig. 5b, FE predictions of the temporal evolution of the normalised crack extensions $\Delta a / a_{\mathrm{s}}$ for $a_{\mathrm{s}}=0.25 \mu \mathrm{m}$ and selected values of $v_{\mathrm{c}}$. These results are for a remote applied stress $\sigma^{\infty}=900 \mathrm{MPa}$ which results in runaway crack growth for the $v_{\mathrm{c}}=100 \mathrm{~ms}^{-1}$ case shown in Fig. 5a. The effect of the initial microcrack velocity is now evident with the crack arresting after a small initial extension for $v_{\mathrm{c}} \leq 2 \mathrm{~ms}^{-1}$, i.e. consistent with our expectation from the Tvergaard and Hutchinson (1992) 
analysis for the limit of $v_{\mathrm{c}} \rightarrow 0$, macroscopic fracture cannot occur for the cohesive and plasticity parameters employed here at a finite $\sigma^{\infty}$.

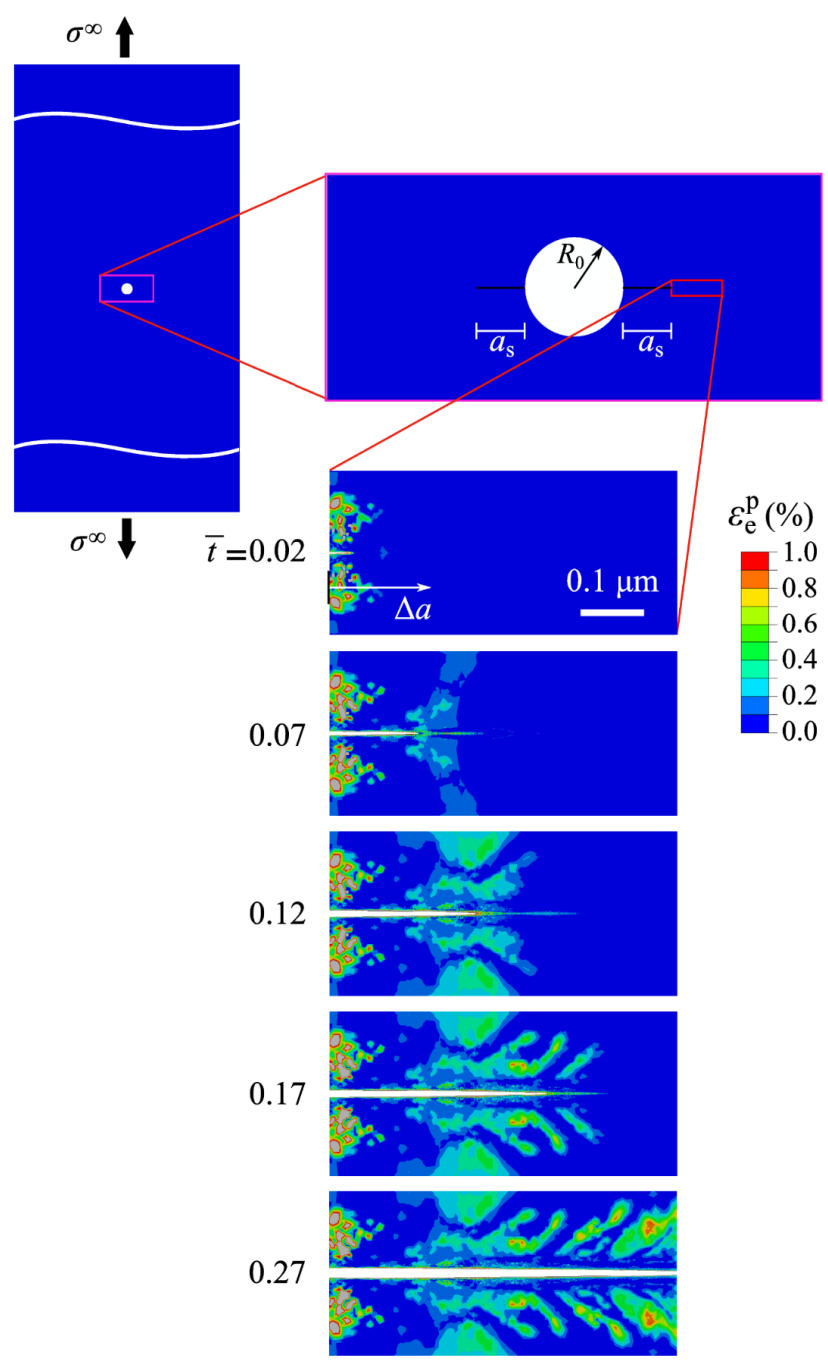

Figure 6: FE predictions of the spatial distribution of the von-Mises effective plastic strain $\varepsilon_{\mathrm{e}}^{\mathrm{p}}$ around the propagating crack-tip for an applied stress $\sigma^{\infty}=900 \mathrm{MPa}$ and a hydrogen-assisted microcrack velocity $v_{\mathrm{c}}=100 \mathrm{~ms}^{-1}$ with $a_{\mathrm{s}}=0.25 \mu \mathrm{m}$ and a cavity of radius $R_{0}=0.15 \mu \mathrm{m}$. Results are shown for 5 selected normalized times $\bar{t} \equiv t \dot{\varepsilon}_{\mathrm{t}}$ where time $t=0$ corresponds to the instant when crack growth is no longer prescribed and the crack has emanated a length $a_{\mathrm{s}}$ from the cavity surface.

For a given microcrack velocity $v_{\mathrm{c}}$ and $a_{\mathrm{s}} / R_{0}$ we define a critical failure stress $\sigma_{\mathrm{f}}$ as the minimum value of $\sigma^{\infty}$ for specimen failure by runaway crack growth. Predictions of the dependence of $\sigma_{\mathrm{f}}$ on the normalised microcrack velocity $v_{\mathrm{c}}$ are included in Fig. 7a for $a_{\mathrm{s}}=$ $0.25 \mu \mathrm{m}$. A double abscissa is included in Fig. 7a where we replot the microcrack velocity in normalised form as $\bar{v}_{\mathrm{c}} \equiv v_{\mathrm{c}} /\left(r_{\mathrm{p}} \dot{\varepsilon}_{\mathrm{t}}\right)$. Intriguingly we observe that the failure strength transitions sharply around $\bar{v}_{\mathrm{c}} \approx 1\left(v_{\mathrm{c}} \approx 0.05 \mathrm{~ms}^{-1}\right)$ and is nearly independent of the microcrack velocity above and below that transition velocity analogous to a ductile-brittle transition. For $\bar{v}_{\mathrm{c}}<1$, the microcrack arrests after minimal additional crack extension beyond the hydrogen-assisted level and cleavage fracture is precluded. Failure is then via tensile plastic necking at an overall tensile plastic strain $\varepsilon^{\mathrm{p}}=1 / n$ with $\sigma_{\mathrm{f}}=\sigma^{\mathrm{UTS}}$. At $\bar{v}_{\mathrm{c}}>1$, the plastic strain-rates $\dot{\varepsilon}^{\mathrm{p}}$ in the immediate vicinity of the crack-tip now exceed $\dot{\varepsilon}_{\mathrm{t}}$, i.e. plasticity is limited by the drag on dislocations due to phonon scattering with the flow strength increasing linearly with $\dot{\varepsilon}^{\text {p }}$. This very strong dependence on plastic strain-rate implies that the material can sustain a crack 
opening stress $T_{\max }=15 \mathrm{GPa}$ at relatively moderate levels of plastic strain as long as $\dot{\varepsilon}^{\mathrm{p}}>\dot{\varepsilon}_{\mathrm{t}}$ and this allows for a quasi-cleavage fracture mode. We shall show in Section 4, that hydrogenassisted cleavage with hydrogen supply from the cavity results in a microcrack velocity $v_{\mathrm{c}} \approx$ $100 \mathrm{~ms}^{-1}\left(\bar{v}_{\mathrm{c}} \approx 2000\right)$ implying that it will permit quasi-cleavage fracture as this velocity is in excess of both the $v_{\text {blunt }}^{\text {crit }} \approx 5.4 \mathrm{~ms}^{-1}$ required to limit dislocation emission and the $v_{\mathrm{c}} \approx$ $0.05 \mathrm{~ms}^{-1}$ required to restrict plasticity in the bulk around the crack-tip. We emphasize that while the transition velocity at which cleavage is precluded due to bulk plasticity around the crack-tip is strongly dependent on $\dot{\varepsilon}_{t}$, there exists a wealth of experimental data on this transition strain-rate with $\dot{\varepsilon}_{\mathrm{t}}=10^{6} \mathrm{~s}^{-1}$ as employed here a direct measurement for 4340 steel.
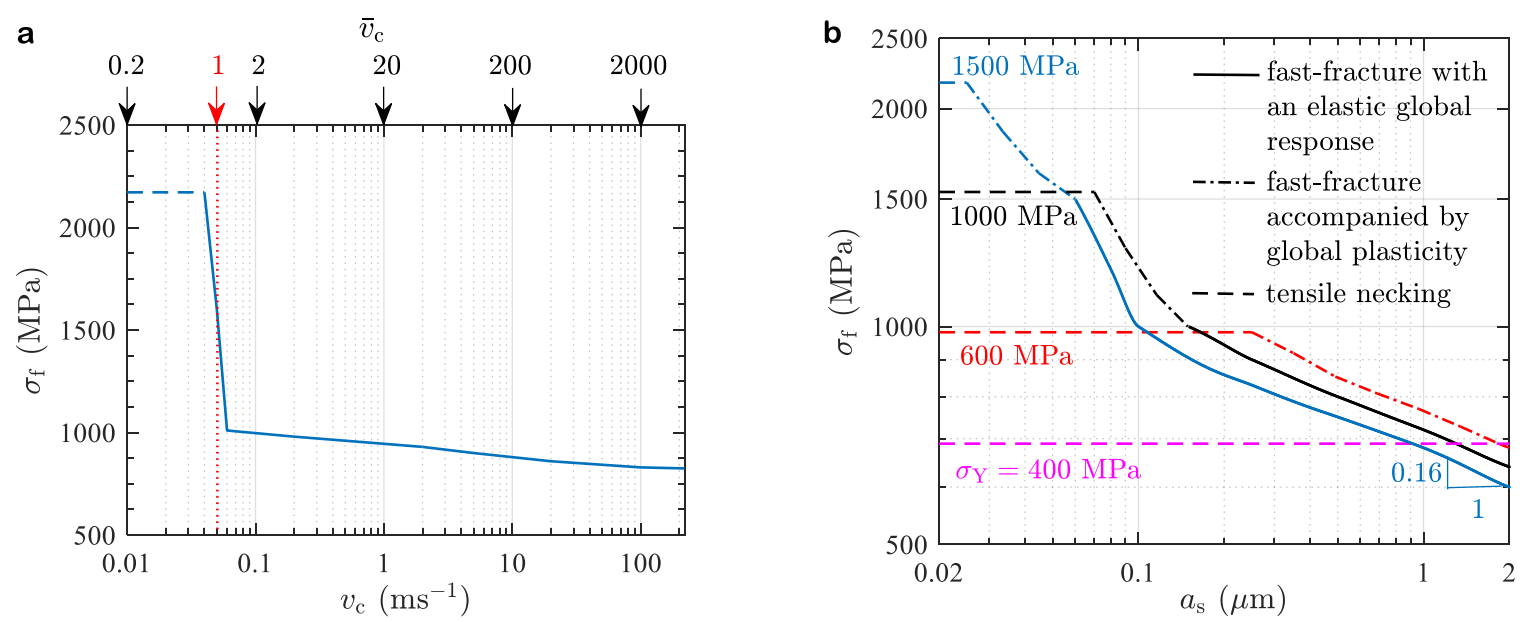

Figure 7: FE predictions of the tensile failure strength $\sigma_{\mathrm{f}}(\mathrm{a})$ as function of the hydrogen-assisted microcrack velocity $v_{\mathrm{c}}$ (a double abscissa is included to also show the corresponding normalised microcrack velocity $\left.\bar{v}_{\mathrm{c}} \equiv v_{\mathrm{c}} /\left(r_{\mathrm{p}} \dot{\varepsilon}_{\mathrm{t}}\right)\right)$ for a microcrack length $a_{\mathrm{s}}=0.25 \mu \mathrm{m}$ and (b) as a function of the microcrack length $a_{s}$ for $v_{\mathrm{c}}=100 \mathrm{~ms}^{-1}$. In (b) predictions are included for three yield strength values in addition to the reference case of $\sigma_{\mathrm{Y}}=1500 \mathrm{MPa}$. Tensile failure by necking rather than brittle crack propagation is indicated by dashed lines in both (a) and (b). All results are for a cavity of radius $R_{0}=$ $0.15 \mu \mathrm{m}$.

A summary of the failure stress $\sigma_{\mathrm{f}}$ as a function of the microcrack length $a_{\mathrm{s}}$ for hydrogenassisted microcrack growth at $v_{\mathrm{c}}=100 \mathrm{~ms}^{-1}$ is included in Fig. $7 \mathrm{~b}\left(\sigma_{\mathrm{f}}\right.$ determined to an accuracy of $\pm 10 \mathrm{MPa}$ ). Three regimes of failure are marked: (i) failure by plastic necking at an overall tensile plastic strain $\varepsilon^{p}=1 / n$ for small microcrack lengths; (ii) at intermediate microcrack lengths failure occurs by fast crack propagation at $\sigma_{\mathrm{f}}>\sigma_{\mathrm{Y}}$ and is thus accompanied by global plasticity and (iii) brittle fracture or quasi-cleavage with no global plasticity for large $a_{\mathrm{s}}$. While the overall regimes in Fig. $7 \mathrm{~b}$ are analogous to what is expected from a Dugdale (1960) and Barenblatt (1962) type analyses there is crucial difference. The Dugdale and Barenblatt models asymptote to the fracture toughness of the solid in the limit of large crack lengths (large $a_{\mathrm{s}}$ in our case) with $\sigma_{\mathrm{f}} \propto 1 / \sqrt{a_{\mathrm{s}}}$. However, it is clear from Fig. 7b that such a scaling is never attained in our analysis where we model macroscopic fracture following hydrogen-assisted fast crack propagation. Rather, the numerical calculations in Fig. 7b suggest that $\sigma_{\mathrm{f}} \propto a_{\mathrm{s}}^{-0.16}$. This is because in our numerical analysis following hydrogen-assisted crack growth, the near-tip $K_{\mathrm{I}}$ is not given by just the remote stress and $a_{\mathrm{s}}$ but requires the solution to the full elastoplastic-dynamic problem of the specific initial/boundary value problem analysed here. The outcome of that solution is a breakdown of the simple static fracture scaling laws and point to the fact that inertial effects play a significant role and therefore we cannot define an effective fracture toughness for failure that initiates from the hydrogen-assisted fast growth of 
a micro-crack. We emphasize here that the results of Fig. $7 \mathrm{~b}$ are relatively insensitive to the choice of $v_{\mathrm{c}}$ for $v_{\mathrm{c}} \gg 0.05 \mathrm{~ms}^{-1}$, i.e. an accurate estimate of $v_{\mathrm{c}}$ is not essential so long as the estimate confirms that $v_{\mathrm{c}} \gg 0.05 \mathrm{~ms}^{-1}$.

The results in Fig. $7 \mathrm{~b}$ can be converted to predictions of $\sigma_{\mathrm{f}}$ versus $c_{\mathrm{L}}$ by connecting the microcrack length $a_{\mathrm{s}}$ to the hydrogen pressure loading $p_{\mathrm{H}}$ (or equivalently $c_{\mathrm{L}}$ by using Eqs. (3.2) and (3.4) to convert the gaseous hydrogen pressure to the lattice hydrogen concentration under equilibrium conditions) through Eq. (3.8). This predicted dependence of $a_{\mathrm{s}}$ on $c_{\mathrm{L}}$ is included in Fig. 8a and then used to replot the failure strength $\sigma_{\mathrm{f}}$ predictions in Fig. $7 \mathrm{~b}$ but now as a function of $c_{\mathrm{L}}$. These $\sigma_{\mathrm{f}}$ versus $c_{\mathrm{L}}$ predictions are also included in Fig. 8a for $\sigma_{\mathrm{f}}<\sigma_{\mathrm{Y}}$, i.e. within the validity of the microcrack length estimate given in Eq. (3.8). The predictions are consistent with a wide body of experimental data (San Marchi and Somerday, 2012) that suggests that high strength steels such as 4340 fail by brittle fracture with no macroscopic plasticity when subjected to relatively high levels of hydrogen loading in the range 0.05 appm $\leq c_{\mathrm{L}} \leq 1$ appm.
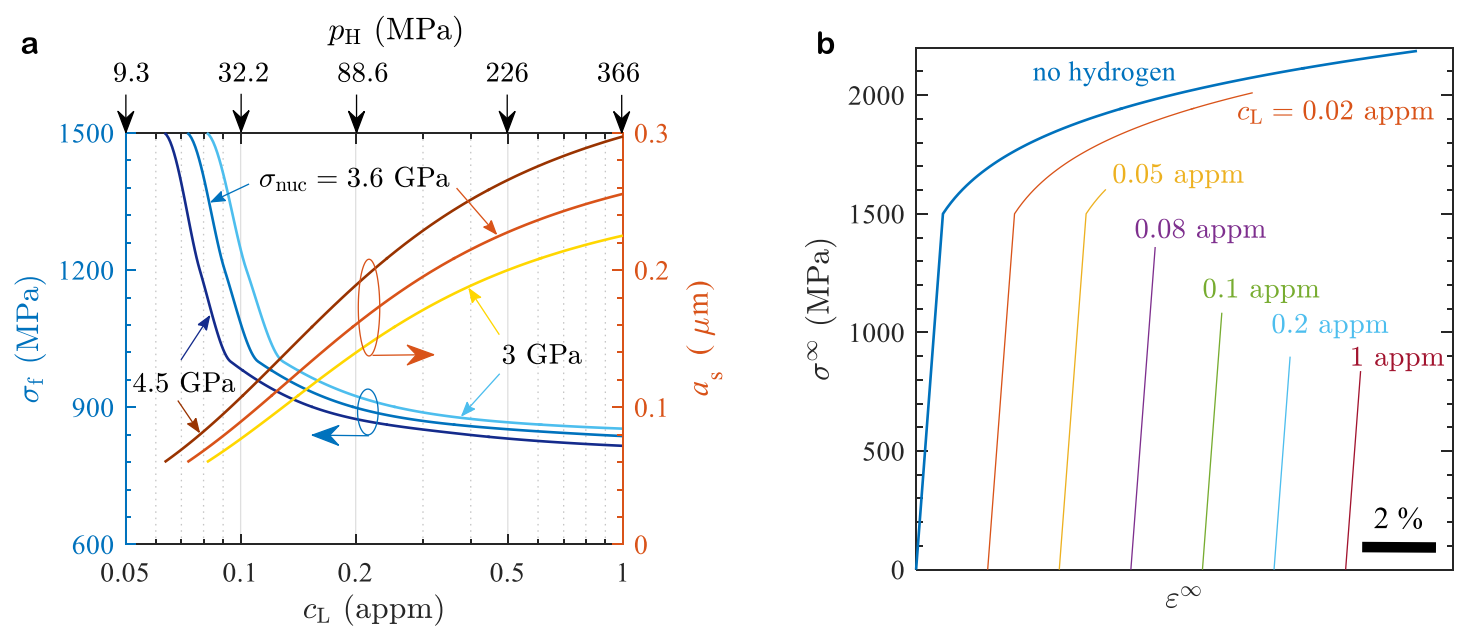

Figure 8: (a) Predictions of the tensile strength $\sigma_{\mathrm{f}}$ and corresponding microcrack size $a_{\mathrm{s}}$ (right-hand $y$-axis) that caused this failure as a function of the lattice hydrogen concentration $c_{\mathrm{L}}$. A double abscissa to show the corresponding hydrogen gas pressure $p_{\mathrm{H}}$ is also included. (b) Selected tensile stress $\sigma^{\infty}$ versus conjugate strain $\varepsilon^{\infty}$ curves for hydrogen concentrations in the range 0 appm $\leq c_{\mathrm{L}} \leq 1 \mathrm{appm}$. In (a) all results are for high levels of hydrogen $c_{\mathrm{L}}$ such that tensile fracture occurs prior to the tensile yield strength being attained while in (b) predictions for lower levels of $c_{\mathrm{L}}$ when failure occurs with $\sigma_{\mathrm{f}}>\sigma_{\mathrm{Y}}$ are also included. In (a) results are shown for three choices of the hydrogen-assisted cleavage nucleation stress $\sigma_{\text {nuc }}$ while in (b) we assume the reference cleavage nucleation stress $\sigma_{\text {nuc }}=3.6 \mathrm{GPa}$.

\subsection{Interpretation of the predictions for hydrogen-assisted brittle fracture}

Predictions of the tensile stress $\sigma^{\infty}$ versus strain $\varepsilon^{\infty}$ response in the limit of an applied strainrate $\dot{\varepsilon}^{\infty} \rightarrow 0$ are included in Fig. 8b. The reference case of loading in the absence of hydrogen is also included with the $\sigma^{\infty}-\varepsilon^{\infty}$ curve terminated at the plastic necking strain of $1 / n$. Here we only consider the case of the high levels of dissolved hydrogen in the range $0.08 \mathrm{appm} \leq$ $c_{\mathrm{L}} \leq 1$ appm such that fracture occurs in the absence of global plasticity. The results for the lower levels of hydrogen where failure is accompanied by global plasticity are discussed in Section 5 .

The dependence of $\sigma_{\mathrm{f}}$ on $c_{\mathrm{L}}$ is summarized in Fig. 8a where a double abscissa is employed to show the dependence of $\sigma_{\mathrm{f}}$ on both $c_{\mathrm{L}}$ and the corresponding pressure $p_{\mathrm{H}}$ of the external hydrogen atmosphere (or equivalently the hydrogen gas pressure in the cavity just prior to 
fracture). In order to understand these predictions, recall that the hydrogen-assisted growth of a nanoscopic crack emanating from the surface of the cavity occurs at a cavity volume $\Delta V_{\text {nuc }}$. While $\Delta V_{\text {nuc }}$ is independent of the hydrogen content $c_{\mathrm{L}}$, the pressure within the cavity and hence the amount of hydrogen within the cavity is strongly dependent on $c_{\mathrm{L}}$ through Eqs. (3.2) and (3.4). Consequently, Eq. (3.8) predicts that the extent of hydrogen-assisted crack growth $a_{\mathrm{s}}$ increases with $c_{\mathrm{L}}$; see Fig. 8a. Given that the fracture strength $\sigma_{\mathrm{f}}$ in turn decreases with increasing $a_{\mathrm{s}}$ (Fig. 7b) it follows that $\sigma_{\mathrm{f}}$ decreases with increasing $c_{\mathrm{L}}$. The sensitivity of $a_{\mathrm{s}}$ to the choice of $\sigma_{\text {nuc }}$ is also indicated in Fig. 8a along with the corresponding predictions of $\sigma_{\mathrm{f}}$ for $\sigma_{\text {nuc }}=3 \mathrm{GPa}, 3.6 \mathrm{GPa}$ and $4.5 \mathrm{GPa}$. Slightly counterintuitively, $\sigma_{\mathrm{f}}$ for a given $c_{\mathrm{L}}$ decreases with increasing $\sigma_{\text {nuc }}$ : with increasing $\sigma_{\text {nuc }}$, the cavity volume $\Delta V_{\text {nuc }}$ increases which in turn drives larger hydrogen-assisted crack extensions $a_{\mathrm{s}}$ and thereby a reduction in $\sigma_{\mathrm{f}}$. Nevertheless, we emphasize that the predictions of $\sigma_{\mathrm{f}}$ are not strongly dependent on $\sigma_{\text {nuc }}$ over the range of values investigated here.
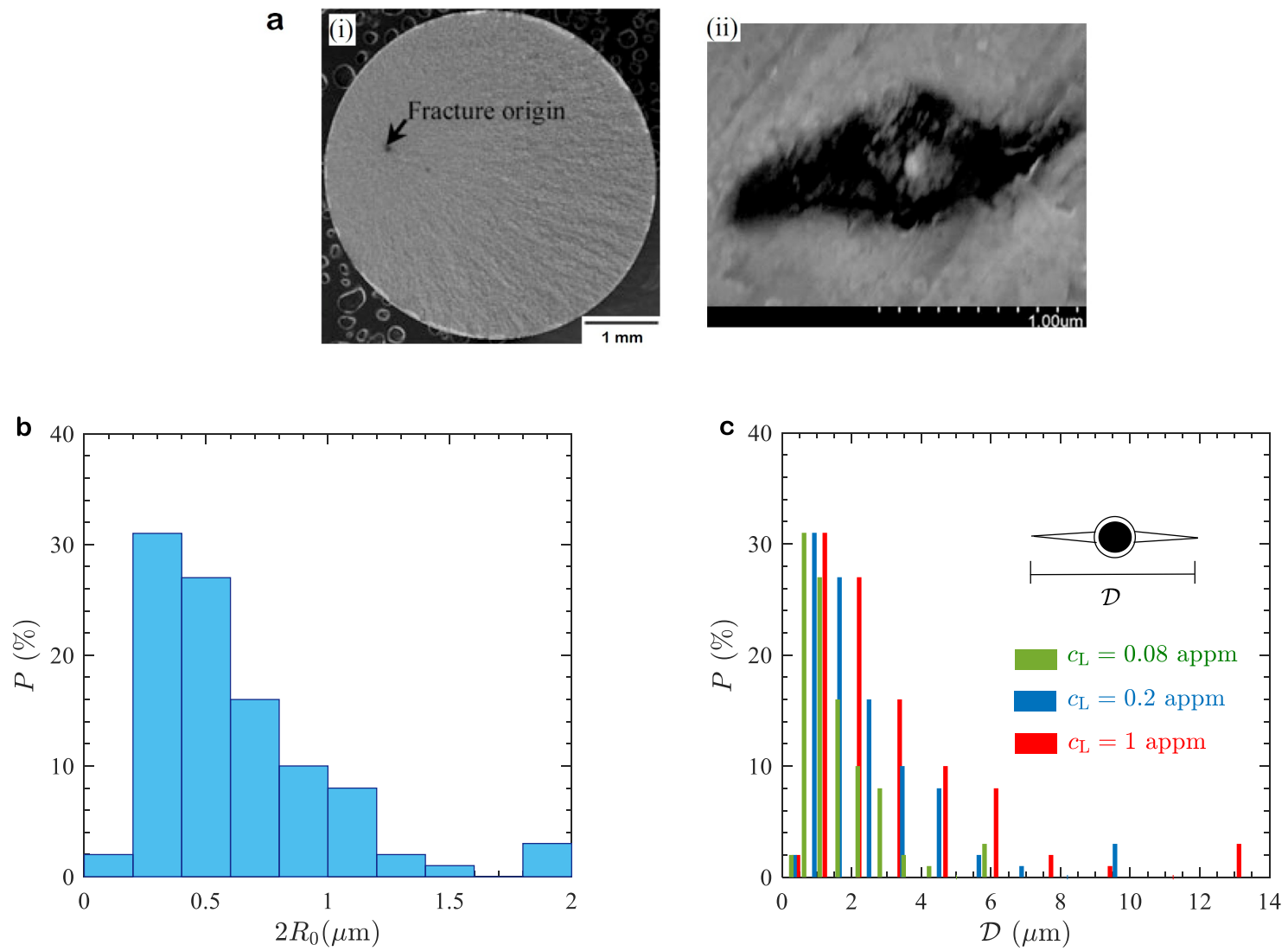

Figure 9: (a) Examples of (i) a fracture surface where failure initiated from an inclusion in a hydrogencharged A485 steel (Fujita and Murakami, 2012) and (ii) hydrogen induced microcracks around carbide particles in a high strength steel (Li et al., 2017). (b) Probability of the diameters $2 R_{0}$ of carbide particles in 4340 steel as reported by Novak et al. (2010). (c) Predictions of the probability of microcrack diameters $\mathcal{D} \equiv 2\left(R_{0}+a_{\mathrm{s}}\right)$ in the 4340 steel investigated by Novak et al. (2010) at selected levels of hydrogen charging $c_{\mathrm{L}}$.

There is extensive experimental data demonstrating that tensile fracture of steels in a hydrogen environment initiates from inclusions; see for example in Fig. 9a a micrograph of the fracture surface of an ASTM A485 steel with a lattice hydrogen concentration $c_{\mathrm{L}}=0.05$ appm (Fujita and Murakami, 2012). In addition to such observations of the fracture surfaces, extensive microcracking of hydrogen-charged specimens subjected to mechanical loading also has been widely reported. For example, Li et al. (2017) observed microcracks that nucleated from 
carbide particles in a hydrogen-charged low alloy steel (Fig. 9a) while Novak et al. (2010) also reported observations of microcracks in their hydrogen-charged 4340 steel. In such observations these microcracks are cracks that have arrested within the specimens and not caused the overall final fracture. In addition to these microcracks, acoustic emissions are also detected for steels loaded in hydrogen environments with the level and frequency of the emissions increasing just prior to the final fracture event (Kameda and Mahon, 1983). A rationalization of all these observations is possible via the hydrogen induced fast-fracture mechanism proposed here. Consider for example, the 4340 steel investigated by Novak et al. (2010) which contains carbide particles with the probability of diameters $P\left(2 R_{0}\right)$ as shown in Fig. 9b. Hydrogen-assisted microcracks grow rapidly from cavities that form around these particles when the local stress $\sigma_{\text {local }}$ attains a value $\sigma_{\text {nuc }}$. Recall that $\sigma_{\text {local }}$ is set by stress concentrations within the specimen such as grain boundary triple junctions and therefore there exists a wide distribution of $\sigma_{\text {local }}$ within the specimen at any given remote applied stress $\sigma^{\infty}$. Thus, hydrogen-assisted crack growth is expected to initiate at different locations within the specimen where $\sigma_{\text {local }}=\Sigma_{\text {nuc }}$ even when the remote stress $\sigma^{\infty}<\sigma_{\mathrm{f}}$. These hydrogen-assisted microcracks will grow by $a_{\mathrm{s}}$ and then arrest as the global stress is insufficient to sustain crack growth after the hydrogen from the cavity feeding them is exhausted. Moreover, in the absence of a rapid hydrogen supply these cracks then blunt by dislocation emission (as the local $K_{\mathrm{I}}$ exceeds $K_{\text {emit }}$ ) and are therefore no longer capable of growing even after hydrogen refills the cavity via diffusive flux from the matrix. We argue that this is the reason for the observation of microcracks for steels loaded in a hydrogen environment and the acoustic emissions are associated with the formation of these microcracks that grow dynamically at $\approx 100 \mathrm{~ms}^{-1}$ via a hydrogen-assisted cleavage mechanism.

The distribution of microcracks sizes can be deduced from our model as follows. Let $\mathcal{D}=$ $2\left(R_{0}+a_{\mathrm{s}}\right)$ denote the diameter of a microcrack emanating from a carbide particle of radius $R_{0}$. Then assuming a wide distribution of $\sigma_{\text {local }}$ within the specimen, the probability of initiating hydrogen-assisted crack growth around carbide particles of all sizes is equal. It therefore follows that the probability of $\mathcal{D}$ equals $P\left(2 R_{0}\right)$. Predictions of the probability $P(\mathcal{D})$ for three levels of hydrogen charging $C_{\mathrm{L}}$ are included in Fig. 9c. Lower levels of hydrogen charging result in a higher probability of small microcracks with the probability distribution tending to spread to larger size microcracks with increasing $c_{\mathrm{L}}$ as $a_{\mathrm{s}}$ increases with increasing $c_{\mathrm{L}}$ through Eq. (3.8). A corollary to these predictions is that the model suggests hydrogenassisted tensile fracture to have a strong statistical nature. In fact, the data presented in Yahya and Pineau (1998) and Ayas et al. (2015) shows a large scatter in the measured strengths and a resulting Weibull modulus in the range $3-4$. Of course, this statistical nature of the fracture process is not included in the numerical predictions of the fracture strength presented here where we have assumed that fracture is dominated by the carbide inclusions of size that are present in the highest numbers, i.e. the $R_{0}=0.15 \mu \mathrm{m}$ carbides in our case. The key drawback of this assumption is that we cannot correlate the fracture strength with the microcrack size distributions shown in Fig. 9c. The extension of our model to account for the statistical nature of the carbide inclusion sizes including the possible emergence of weakest-link statistics remains a topic for future investigations.

\section{Atomistic calculations of hydrogen-assisted fast crack growth (Stage-A)}

The above predictions show excellent overall agreement with observations of the tensile response of 4340 steel specimens in a hydrogen environment. One of the key ingredients to these predictions is the claim that hydrogen gas in a cavity within the material drives a fast- 
growing cleavage microcrack - this microcrack runs for a length $a_{\mathrm{s}}$ until this hydrogen supply is exhausted. Here we shall justify this claim via atomistic calculations in three steps:

(i) First, we demonstrate that, given a Fe hydride at the crack-tip, mode-I loading results in cleavage at $G_{\mathrm{I}}=4.2 \mathrm{~J} \mathrm{~m}^{-2}$ corresponding to a $K_{\mathrm{I}} \approx 1 \mathrm{MPa} \sqrt{\mathrm{m}}$. By contrast, dislocation emission and crack-tip blunting occur in the absence of the hydride. This result is in line with the overall findings of Song and Curtin (2013).

(ii) Second, we establish that crack-tip hydrides are thermodynamically stable at an applied $G_{\mathrm{I}}$ below that required for initiating dislocation emission in the system absent $\mathrm{H}$ even at relatively low levels of the lattice hydrogen concentration $c_{\mathrm{L}}$. Thus, they not only prevent early dislocation emission but also exist at the $G_{\mathrm{I}}$ levels required to initiate their cleavage.

(iii) Finally, we show that both the energy barrier for the entry of $\mathrm{H}$ into the loaded crack-tip and the barrier for $\mathrm{H}$ diffusion over a nanometer scale region ahead of the crack-tip are relatively small. These small barriers in turn imply that the hydride is rapidly replenished at the crack-tip by the hydrogen supplied from within the cavity. This results in a hydrogen-assisted cleavage crack propagating at a velocity $>$ $100 \mathrm{~ms}^{-1}$.

These findings are based on both quasi-static and molecular dynamics (MD) calculations performed using interactions between iron and hydrogen atoms described by an Embedded Atom Potential (EAM) developed by Ramasubramaniam et al. (2009). Specifically, we employed their potential B and confirmed using tests similar to those reported by Song and Curtin (2013) that, both at zero pressure and tensile strains consistent with the strains at the crack-tip, we obtained no unphysical aggregation of $\mathrm{H}$. The intrinsic response of the Fe lattice as described by this EAM potential is discussed in Appendix B and will be used not only to interpret the deformation and fracture mechanisms at the crack-tip but also help quantify results in terms of macro loading parameters.

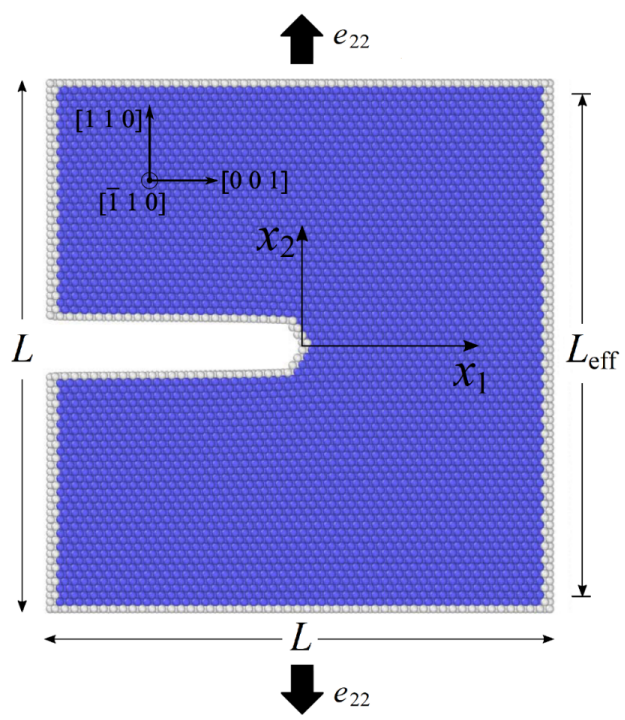

Figure 10: Sketch of the atomistically detailed quasi two-dimensional model for investigating the hydrogen-assisted growth of a microcrack. A crack with a crack plane normal (110) and crack front direction [110] is generated in BCC Fe by removing 6 half planes and loaded in mode-I by applying a strain $e_{22}$ in the $x_{2}$-direction. The co-ordinate system centred at the crack-tip is also indicated.

The problem we considered is a cracked quasi-two-dimensional (2D) BCC Fe system with a crack in the (110) plane and the crack front in the [110] direction loaded in mode-I (Fig. 10). 
(We recognise that while a full 3D calculation can introduce new crack-tip mechanisms for both cleavage and dislocation emission we do not believe our quasi-2D approximation with one unit cell in the $x_{3}$ - direction introduces significant errors in this context as our results for cleavage and dislocation emission are consistent with previous findings of Song and Curtin (2013) that used a significantly thicker system.) The crack-tip is taken as the origin of a Cartesian co-ordinate system with the crack plane normal aligned along the $x_{2}$-direction and vanishing periodic strains enforced along the [110] crack front direction that is aligned with the $x_{3}$-axis (i.e. closely analogous to plane strain conditions). The cracked configuration in Fig. 10 comprised a block of height and length $L \approx 15 \mathrm{~nm}$, i.e. consistent with the threshold system size suggested by Andric and Curtin (2019). The crack with a crack plane (110) and crack front direction [110] was generated by removing 6 half-planes ${ }^{2}$ of atoms and subjecting the system to mode-I tensile loading. Symmetry of the crystal in this orientation implied that mode-I loading could be achieved by displacing all atoms in the $x_{2}$ - direction via the relation $u_{2}^{(i)}=e_{22} x_{2}^{(i)}$, where $e_{22}$ is the imposed strain while $u_{2}^{(i)}$ is the displacement of atom $(i)$ with $x_{2}$ co-ordinate $x_{2}^{(i)}$. Subsequently, all atoms of the system (excluding atoms within $0.8 \mathrm{~nm}$ of the top and bottom surfaces) were relaxed to their minimum energy states and this either provided the quasi-static state of the system strained to $e_{22}$ or a starting position from which a MD calculation was conducted. All results are presented in terms of the applied mode-I energy release rate $G_{\mathrm{I}}=\Phi\left(e_{22}\right) L_{\text {eff }}$ where $\Phi\left(e_{22}\right)$ is the strain energy density far upstream from the crack-tip and thus given by the BCC lattice strain energy plotted in Fig. B.1a in Appendix B. Here $L_{\text {eff }}$ is the effective height of the system discounting the $0.8 \mathrm{~nm}$ regions at the top and the bottom where the atom positions were not allowed to naturally evolve (we always applied strains $e_{22}<0.14$ and thus no phase transformations occurred in the far-field from the cracktip; see Appendix B for a discussion on stress-induced phase transformations of the Fe lattice). The corresponding mode-I stress intensity factor is given by $K_{\mathrm{I}}=\sqrt{G_{\mathrm{I}} / C_{\mathrm{G}}}$, where for this crack orientation, $C_{\mathrm{G}}=3.87 \mathrm{~Pa}(\mathrm{MPa})^{-2}$ with $K_{\mathrm{I}}$ and $G_{\mathrm{I}}$ in the commonly employed units of $\mathrm{MPa} \sqrt{\mathrm{m}}$ and $\mathrm{J} \mathrm{m}^{-2}$, respectively; see Appendix B for details including the anisotropic elastic constants of BCC Fe for the EAM potential employed here. Note that $G_{\mathrm{I}}$ as calculated here is the non-linear energy release rate that is usually denoted as $J_{\mathrm{I}}$ in the continuum mechanics literature. However, to remain consistent notation typically used in atomistic calculations we shall refer to it as $G_{\mathrm{I}}$ with the understanding that relations such as $K_{\mathrm{I}}=\sqrt{G_{\mathrm{I}} / C_{\mathrm{G}}}$ hold equally if $G_{\mathrm{I}}$ is the non-linear energy release rate.

\subsection{Hydride induced cleavage}

To investigate crack propagation, we report MD calculations at $T=293 \mathrm{~K}$ using a time step of $1 \mathrm{fs}$, and Langevin thermostat with a friction constant of $\approx 1 \mathrm{ps}^{-1}$ (spot checks to confirm convergence of our MD calculations were also performed using a time step of $0.5 \mathrm{fs}$ ). In all cases the system was first loaded quasi-statically to a relatively low $G_{\mathrm{I}}$ level and then loading continued in the MD setting by incrementing positions of all atoms $(i)$ by $\Delta u_{2}^{(i)}=\Delta e_{22} x_{2}^{(i)}$, where $\Delta e_{22}=10^{-5}$ every $1 \mathrm{ps}$. This thus corresponds to an imposed average strain rate $\dot{e}_{22}=$ $10^{7} \mathrm{~s}^{-1}$ : while this is an unrealistically high loading rate in terms of experimental conditions it is sufficiently low to allow the system to explore barriers for the phenomena of interest, viz. dislocation emission, phase transformations and cleavage. We consider three cases of the

\footnotetext{
${ }^{2}$ Our aim here is to investigate a crack growth mechanism that is robust, i.e. not reliant completely on an atomistically sharp crack but rather a cleavage mechanism that persists despite some limited blunting by dislocation emission. Nevertheless, we confirmed that the results presented here remain unchanged for configurations with 2, 4, 6 and 8 half-planes removed to form the notch. Thus, to keep the notation consistent with the previous discussion we shall subsequently refer to this notch as a crack.
} 
system under mode-I loading as described above: (i) a clean BCC Fe system with no H; (ii) the system where the crack flanks have full coverage (1 ML) of $\mathrm{H}$ but no $\mathrm{H}$ within the lattice and (iii) a system with a hydride in $\sim 1.5 \mathrm{~nm}$ region ahead of the crack-tip in addition to $1 \mathrm{ML} \mathrm{H}$ coverage of the crack flanks.

a

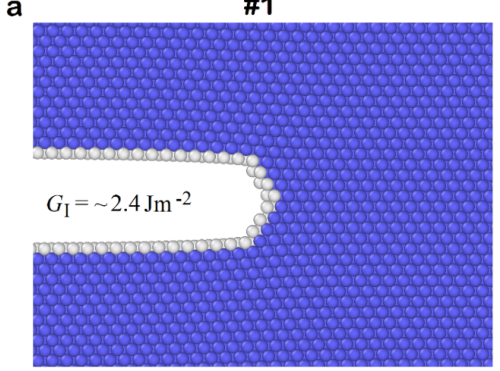

b
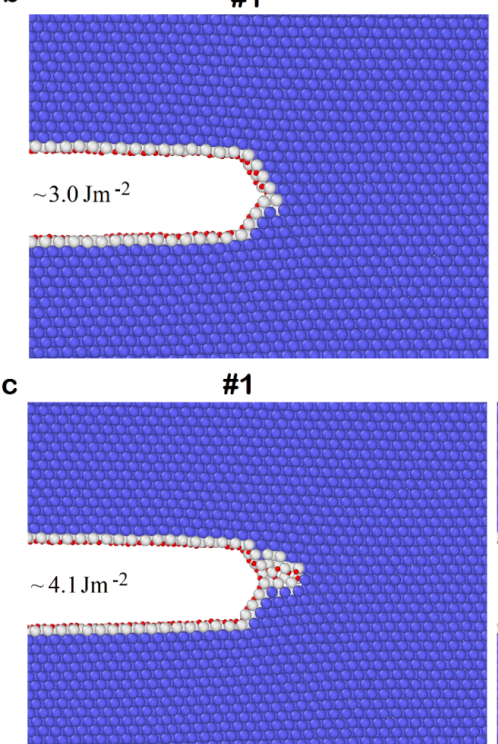

d

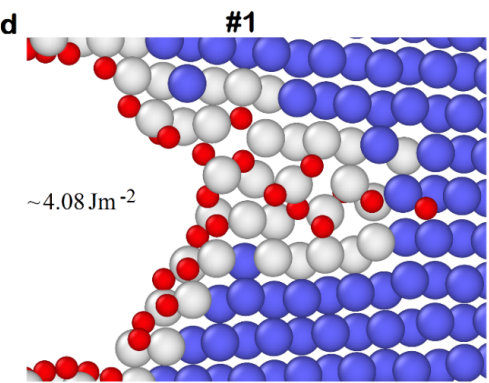

\#2

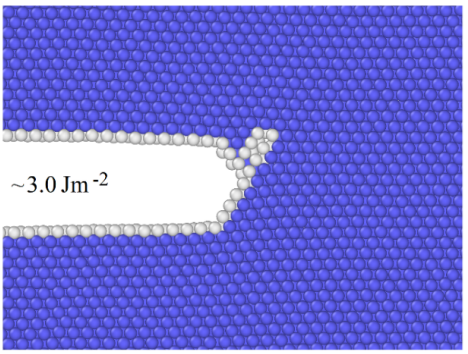

\#2

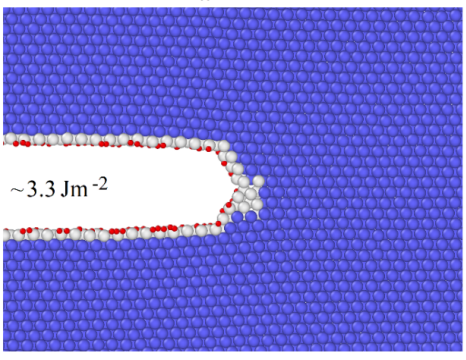

\#2

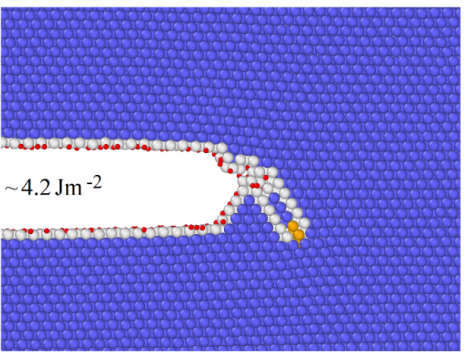

\#2

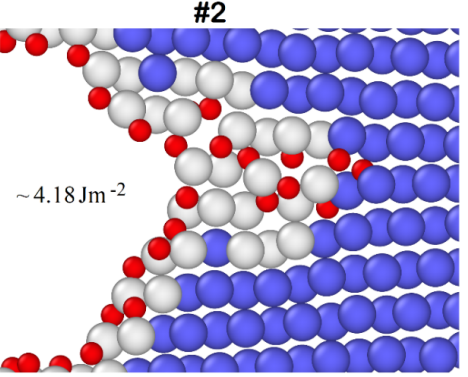

\#3

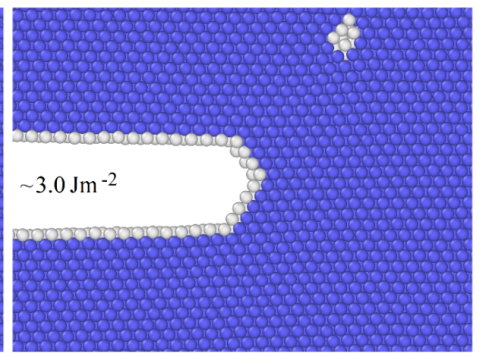

\#3

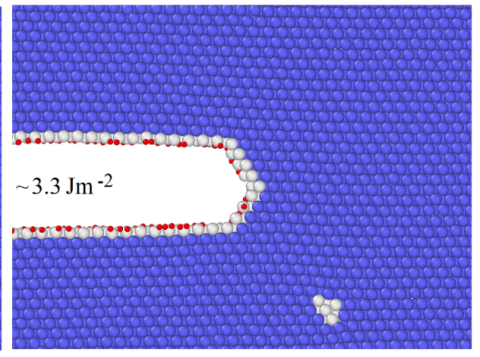

\#3

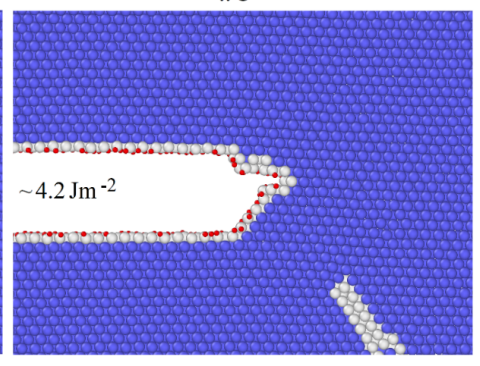

\#3

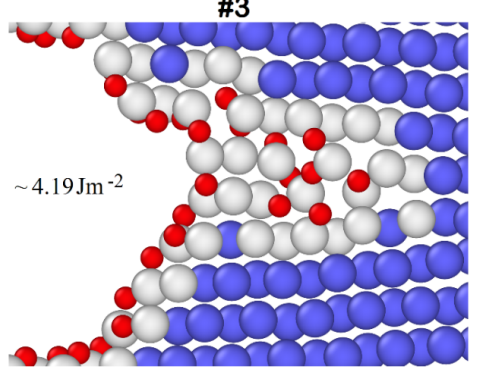

Figure 11: Snapshots of the system from MD calculations $(T=293 \mathrm{~K})$ at selected levels of applied $G_{\mathrm{I}}$. (a) The clean system absent $\mathrm{H}$, (b) the system with $1 \mathrm{ML}$ (Monolayer) $\mathrm{H}$ coverage only on the crack flanks and (c) the system with a hydride around the crack-tip in addition to the 1 ML H coverage on the crack flanks. (d) Close-up snapshots of the crack-tip hydride at $G_{\mathrm{I}} \approx 4.1 \mathrm{~J} \mathrm{~m}^{-2}$ to illustrate its fluctuating nature. Fe atoms are coloured using a common neighbour analysis with blue indicating the BCC phase and white denoting an unknown coordination while the $\mathrm{H}$ atoms (not drawn to-scale) are coloured red. The cores of the emitted dislocations are seen as having atoms with an unknown coordination.

Snapshots of the clean system (i.e. absent $H$ ) at selected levels of applied $G_{\text {I }}$ are shown in Fig. 11a. The system is stable with no clear visible changes seen around the crack-tip until $G_{\mathrm{I}} \approx$ $3.0 \mathrm{~J} \mathrm{~m}^{-2}$ when a dislocation is nucleated at the crack-tip. This dislocation moves rapidly from the tip (snapshots \#2 and \#3 are at approximately the same level of applied $G_{\mathrm{I}}$ ) resulting in the 
blunting of the tip by one atomic spacing. This emission will then continue uninhibited resulting in blunting of the crack-tip. The high affinity of $\mathrm{H}$ to surfaces of Fe implies that under equilibrium conditions (even in the absence of mechanical loading), all surface $\mathrm{H}$ sites are expected to be fully occupied for any level of $c_{\mathrm{L}}$ at which embrittlement is observed. The results in Fig. $11 \mathrm{~b}$ show that full $\mathrm{H}$ coverage of the crack flanks delays dislocation emission which now occurs at $G_{\mathrm{I}} \approx 3.3 \mathrm{~J} \mathrm{~m}^{-2}$ but again emission is preferred over cleavage. Finally consider the case where a hydride is present in approximately a $1.25 \mathrm{~nm}$ region ahead of the crack-tip. Hydrides of $\mathrm{Fe}$ are thermodynamically unstable at $T=293 \mathrm{~K}$ in the absence of large tensile stresses. Thus, in this case we first quasi-statically loaded the clean system to $G_{\mathrm{I}} \approx 4.1 \mathrm{~J} \mathrm{~m}^{-2}$ and then introduced $\mathrm{H}$ atoms both on the crack flanks and $15 \mathrm{H}$ atoms ahead of the crack-tip. The MD simulation was then commenced with no additional loading and the system locally equilibrated itself by ejecting $3 \mathrm{H}$ atoms within $1 \mathrm{ps}$. This equilibrated system with the cracktip hydride is shown in snapshot \#1 in Fig. 11c. We shall show in Section 4.2 that the system with such a large $\mathrm{H}$ concentration ahead of the crack-tip is thermodynamically stable with respect to the remote lattice hydrogen even at low levels of $c_{\mathrm{L}}$ and here we investigate the effect of this hydride on the response of the crack-tip to further loading. Now unlike the previous two cases the crack-tip loaded to $G_{\mathrm{I}} \approx 4.1 \mathrm{~J} \mathrm{~m}^{-2}$ is stable at $T=293 \mathrm{~K}$ when previously uncontrolled dislocation emission occurred at much lower loads. The onset of a dislocation emission event commences at $G_{\mathrm{I}} \approx 4.2 \mathrm{~J} \mathrm{~m}^{-2}$ (snapshot \#2 in Fig. 11c) but the emission is accompanied by cleavage as seen in snapshot \#3 in Fig. 11c. This result for a crack on the (110) plane is very much in line with the findings of Song and Curtin (2013) for cracks on the (111) plane where they too concluded that crack-tip hydrides promote crack propagation by cleavage. As an aside, it is worth pointing out that while we refer to the material with the high $\mathrm{H}$ concentration at the crack-tip as a hydride it behaves unlike normal solids. In particular, the $\mathrm{H}$ within the material is highly mobile with the consequence that the atomic structure is fast fluctuating at $T=293 \mathrm{~K}$. We illustrate this by showing three snapshots of this hydride from the MD simulations at a load $G_{\mathrm{I}} \approx 4.1 \mathrm{~J} \mathrm{~m}^{-2}$. Even over the short MD timescales the hydride fluctuates between significantly different structures and in that respect, it may more aptly be referred to as a dynamic hydride. We defer a detailed investigation of the precise nature of this material comprising $\mathrm{Fe}$ with a high $\mathrm{H}$ concentration (i.e. $\mathrm{FeH}_{y}$ where $y \approx 1-3$ ) to future studies.

An important point to note here is that the surface energy of two Fe (110) surfaces with full $\mathrm{H}$ coverage is predicted to be $1.18-1.28 \mathrm{~J} \mathrm{~m}^{-2}$ by the EAM potential employed here (Appendix B). However, in Fig. 11c we see that the hydride ahead of a crack-tip cleaves at $G_{\mathrm{I}} \approx 4.2 \mathrm{~J} \mathrm{~m}^{-2}$ to form two Fe (110) surfaces with full $\mathrm{H}$ coverage, i.e. a much higher energy is required to cause cleavage fracture. The discrepancy is partially due to the fact that the emitted dislocation (Fig. 11c) absorbed some energy but of course the crack was stable even at $G_{\mathrm{I}} \approx 4 \mathrm{~J} \mathrm{~m}^{-2}$ when there was no dislocation emission. Thus, this discrepancy is mainly attributed to the fact that cleavage involves not only the work required to create new hydrogen covered Fe surfaces but also the work associated with the loss of entropy of the lattice hydrogen (i.e. the difference in the entropy between the hydrogen in the lattice and the hydrogen on the fractured surfaces). This result has significant implications on continuum cohesive zone models for hydrogen embrittlement that assume that the reduction in surface energy due to hydrogen coverage directly sets the hydrogen-mediated fracture energy; see for example Serebrinsky et al. (2004) and Martínez-Pañeda et al. (2016). Importantly, similar to Song and Curtin (2013), we conclude that the main role of $\mathrm{H}$ is in inhibiting dislocation emission and thereby permitting cleavage rather than a reduction in the fracture energy as assumed in the cohesive zone models (the fracture energy of pure Fe (110) based on the surface energy is $\sim 3.2 \mathrm{~J} \mathrm{~m}^{-2}$ using this EAM potential; see Appendix B). 


\subsection{Stress concentrations stabilise a crack-tip hydride}

In Section 4.1, we have shown that a Fe hydride at the crack-tip promotes cleavage and stabilises against dislocation emission. However, in those calculations the hydride was created by introducing $\mathrm{H}$ atoms around the crack-tip with the MD simulations ensuring that the $\mathrm{H}$ atoms attained a local equilibrium. Nevertheless, the MD simulations are unable to ascertain the conditions, in terms of the applied load and far-field lattice hydrogen concentrations $c_{\mathrm{L}}$ (or equivalently pressure of gaseous $\mathrm{H}_{2}$ in the cavity), under which the crack-tip hydrides are in global thermodynamic equilibrium. Here we shall analyse the stability of crack-tip hydrides and show:

(i) Crack-tip hydrides are stable even below $G_{\mathrm{I}} \approx 3 \mathrm{~J} \mathrm{~m}^{-2}$ at which dislocation emission occurs in the clean system. Thus, the hydrides can inhibit dislocation emission which occurs at $G_{\mathrm{I}} \approx 3 \mathrm{~J} \mathrm{~m}^{-2}$ in the clean system (Fig. 11a).

(ii) The formation of these hydrides is promoted by a stress-induced BCC to HCP phase transformation at the crack-tip that enhances the binding affinity of $\mathrm{H}$. This also results in the size of the stable crack-tip hydrides increasing with increasing $G_{\mathrm{I}}$.

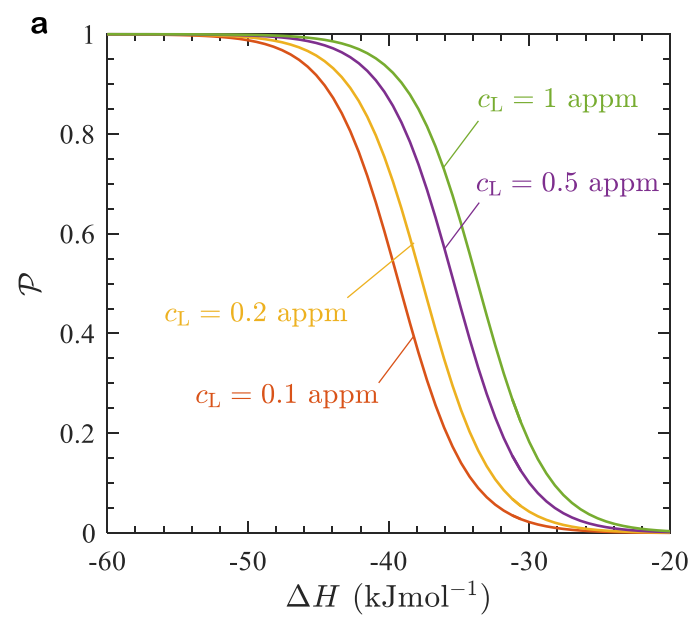

b

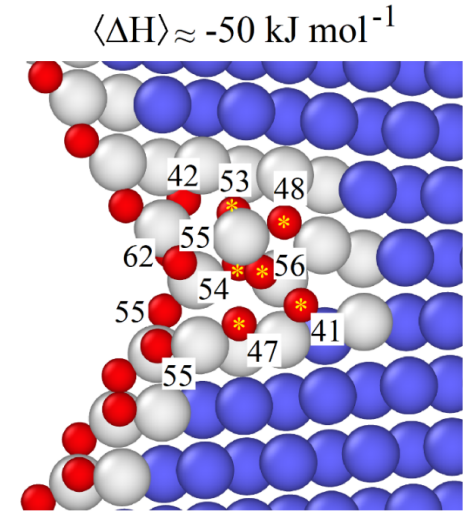

C

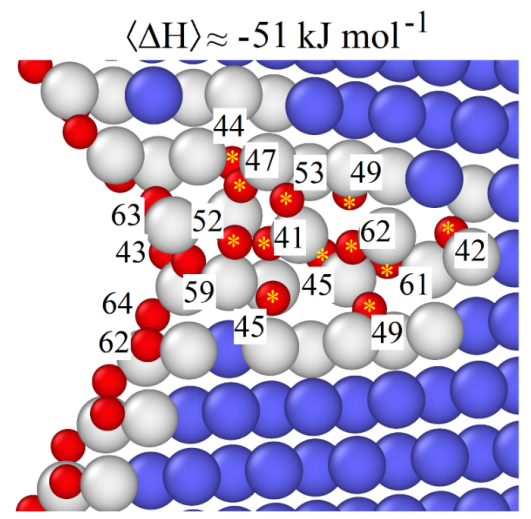

Figure 12: (a) The probability $\mathcal{P}$ of occupancy of a site by a $\mathrm{H}$ atom as a function of the binding energy $\Delta H$ of that site. Results are shown for a system at $T=293 \mathrm{~K}$ and selected values of the lattice hydrogen concentration $c_{\mathrm{L}}$ in the range where embrittlement is typically observed. The binding energies of individual $\mathrm{H}$ atoms in (b) a small hydride loaded to $G_{\mathrm{I}} \approx 3 \mathrm{~J} \mathrm{~m}^{-2}$ and (c) a larger hydride loaded to $G_{\mathrm{I}} \approx 4.1 \mathrm{~J} \mathrm{~m}^{-2}$. In (b) and (c) the binding energies of the individual $\mathrm{H}$ atoms are marked (the values shown are $-\Delta H$ in units of $\mathrm{kJ} \mathrm{mol}^{-1}$ ) along with the average binding energy $\langle\Delta H\rangle$ of the entire hydride (the $\mathrm{H}$ atoms defined to be part of the hydride are indicated by a $*$ ). Fe atoms are coloured using a common neighbour analysis with blue indicating the BCC phase and white denoting an unknown coordination while the $\mathrm{H}$ atoms (not drawn to-scale) are coloured red. 
The stability of a hydride is best characterised in terms of the probability $\mathcal{P}$ of the $\mathrm{H}$ atoms to be present around the crack-tip for the specified level of mechanical loading and remote concentration $c_{\mathrm{L}}$ of lattice hydrogen. The occupancy $\theta$ of sites with a binding energy $\Delta H$ is specified by the Oriani (1970) analysis and is equivalent to the probability $\mathcal{P}$ that the sites are occupied given by

$$
\mathcal{P}=\frac{\theta_{\mathrm{L}} \exp \left(-\frac{\Delta H}{R T}\right)}{1+\theta_{\mathrm{L}} \exp \left(-\frac{\Delta H}{R T}\right)},
$$

where $\theta_{\mathrm{L}}=10^{-6} c_{\mathrm{L}}$. This probability is plotted in Fig. 12a as a function of $\Delta H$ at $T=293 \mathrm{~K}$ for choices of $c_{\mathrm{L}}$ in the range $0.1 \mathrm{appm} \leq c_{L} \leq 1 \mathrm{appm}$ that represents the spectrum of $c_{\mathrm{L}}$ values known to embrittle 4340 steel. Clearly, the probability $\mathcal{P}>50 \%$ for $\Delta H \leq$ $-40 \mathrm{~kJ} \mathrm{~mol}^{-1}$ and approaches $100 \%$ for $\Delta H \leq-50 \mathrm{~kJ} \mathrm{~mol}^{-1}$ even for $c_{\mathrm{L}}=0.1 \mathrm{appm}$, i.e. all sites with binding $\Delta H \leq-40 \mathrm{~kJ} \mathrm{~mol}^{-1}$ can be thought to be occupied under equilibrium conditions over the range of lattice hydrogen concentrations of interest. It now remains to investigate the stability of hydrides in this context.

Consider a crack-tip hydride comprising $n \mathrm{H}$ atoms loaded to a level $G_{\mathrm{I}}$. The binding energy $\Delta H_{i}$ of the $i^{\text {th }} \mathrm{H}$ atom given the presence of the remaining $(n-1)$ hydride $\mathrm{H}$ atoms is then defined as

$$
\Delta H_{i} \equiv N_{\mathrm{A}}\left[E_{\mathrm{ref}}-\left(E_{i}+E_{0}\right)\right]
$$

where $E_{i}$ is the energy of the relaxed system absent this $i^{\text {th }} \mathrm{H}$ atom and $N_{\mathrm{A}}$ is the Avogadro constant. Here $E_{\text {ref }}$ denotes the energy of the relaxed system with all $\mathrm{H}$ atoms (surface plus hydride) being present while $E_{0}$ is the solution energy of a $\mathrm{H}$ atom in bulk unstressed BCC Fe (i.e. $E_{0} \equiv$ energy of system comprising $\mathrm{H}$ in Fe lattice - energy of Fe lattice absent $\mathrm{H}-(1 / 2)$ energy of $\mathrm{H}_{2}$ molecule in vacuum). These binding energies are marked in Fig. $12 \mathrm{~b}$ for a hydride structure subjected to $G_{\mathrm{I}} \approx 3 \mathrm{~J} \mathrm{~m}^{-2}$ : the fact that $\Delta H \leq-41 \mathrm{~kJ} \mathrm{~mol}^{-1}$ for all $\mathrm{H}$ atoms implies that the probability of $\mathrm{H}$ atoms migrating away from the crack-tip is small at $T=293 \mathrm{~K}$ and this level of applied mechanical load. Another measure of stability of the hydride is the probability that all the $n \mathrm{H}$ atoms come together to form the hydride. This is characterised by the average binding energy of all the hydride $\mathrm{H}$ atoms (the $\mathrm{H}$ atoms defined to be part of the hydride are indicated in Fig. $12 \mathrm{~b}$ and exclude the surface $\mathrm{H}$ atoms). This binding energy is defined as

$$
\langle\Delta H\rangle \equiv \frac{N_{\mathrm{A}}\left[E_{\mathrm{ref}}-\left(E_{n}+n E_{0}\right)\right]}{n},
$$

where $E_{n}$ is the energy of the system absent the $n \mathrm{H}$ atoms of hydride. At $G_{\mathrm{I}} \approx 3 \mathrm{~J} \mathrm{~m}^{-2},\langle\Delta H\rangle \approx$ $-50 \mathrm{~kJ} \mathrm{~mol}^{-1}$. These results taken together suggest that hydride shown in Fig. 12b is stable at $T=293 \mathrm{~K}$ with an applied load $G_{\mathrm{I}} \approx 3 \mathrm{~J} \mathrm{~m}^{-2}$ for all $c_{\mathrm{L}} \geq 0.1 \mathrm{appm}$. We note in passing that the mean value of the binding energies of the individual hydride atoms marked in Fig. $12 \mathrm{~b}$ is also $\approx-50 \mathrm{~kJ} \mathrm{~mol}^{-1}$ and thus it suffices to just consider these individual binding energies to determine the stability of the hydride. Next consider a larger hydride shown in Fig. $12 \mathrm{c}$ and loaded to $G_{\mathrm{I}} \approx 4.1 \mathrm{~J} \mathrm{~m}^{-2}$. The individual binding energies of the $\mathrm{H}$ atoms are again labelled with the average binding energy of the hydride $\mathrm{H}$ atoms (labelled in Fig. 12c) $\langle\Delta H\rangle \approx$ $-51 \mathrm{~kJ} \mathrm{~mol}^{-1}$ (again the mean value of the binding energies of the individual hydride atoms $\approx\langle\Delta H\rangle)$. The individual binding energies in Fig. 12c along with $\langle\Delta H\rangle$ both clearly show that this larger hydride is stable at $G_{\mathrm{I}} \approx 4.1 \mathrm{~J} \mathrm{~m}^{-2}$ for all $c_{\mathrm{L}} \geq 0.1 \mathrm{appm}$. 
These calculations have clearly demonstrated the existence of stable hydrides under loads to both prevent dislocation emission and induce cleavage, i.e. the hydride in the MD calculations of Fig. 11c is in equilibrium with the far-field lattice hydrogen concentration or equivalently the gaseous $\mathrm{H}_{2}$ in the cavity. An equivalent stability analysis was also reported by Song and Curtin (2010) for $\mathrm{H}$ at crack-tips in Nickel. However, this fact that a state with such a high $\mathrm{H}$ concentration at the crack-tip is thermodynamically stable is surprising based on commonly employed continuum analyses (Sofronis and McMeeking, 1989). In that analysis, the occupancy $\theta_{\text {tip }}$ of crack-tip hydrogen sites subjected to a hydrostatic stress $\sigma_{\mathrm{m}}$ is estimated using Eqs. (1.2) and (1.3) with the binding energy of crack-tip $H$ sites taken to be $\Delta H_{\text {tip }}=$ $-\sigma_{\mathrm{m}} V_{\mathrm{H}}$. Here $V_{\mathrm{H}}$ is the molar volume of interstitial $\mathrm{H}$ in an unstressed BCC Fe lattice, i.e. $V_{\mathrm{H}}=$ $2 \times 10^{-6} \mathrm{~m}^{3} \mathrm{~mol}^{-1}$ (Hirth, 1980). Then setting $\sigma_{\mathrm{m}}=10 \mathrm{GPa}$ (the maximum tensile stress sustained by the BCC phase loaded in the [110] direction; see Fig. B.1b in Appendix B) it follows that $\theta_{\text {tip }} \approx 3 \times 10^{-3}$ at $T=293 \mathrm{~K}$ even for $\mathrm{c}_{\mathrm{L}}=1$ appm $\left(\right.$ i.e. $\left.\theta_{\mathrm{L}}=10^{-6}\right)$. Given that it is reasonable to assume that there is approximately one $\mathrm{H}$ site associated with each Fe atom around the crack tip, this analysis predicts a $\mathrm{H}$ atom to be present only for about every $300 \mathrm{Fe}$ atoms, i.e. predicts that hydride structures such as those in Fig. 12 are not thermodynamically stable. We now proceed to investigate the mechanism that has been neglected in this continuum analysis and allows the formation of the hydride.

$\# 1$

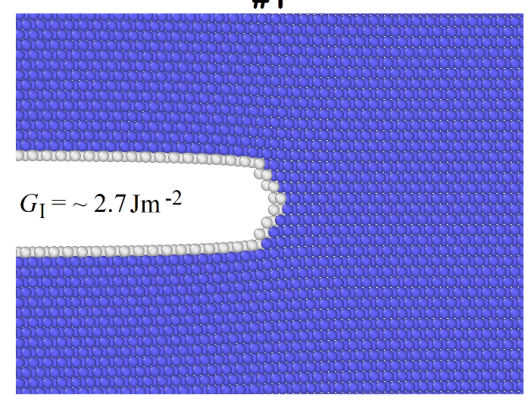

$\# 4$

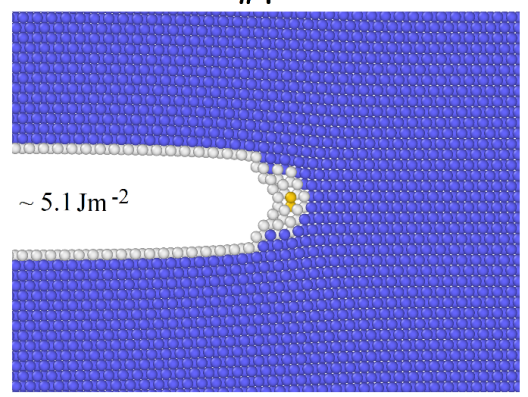

\#2

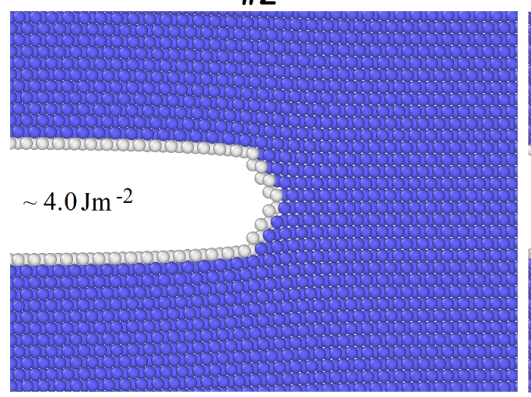

\#5

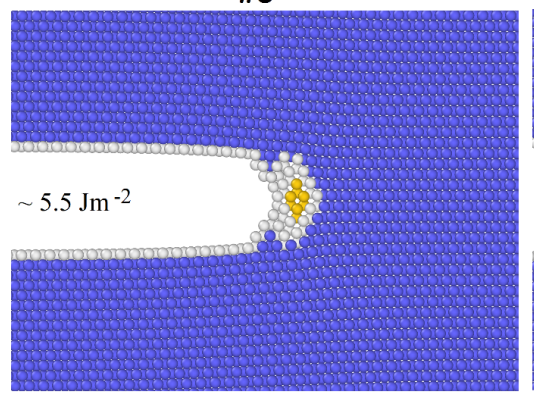

\#3

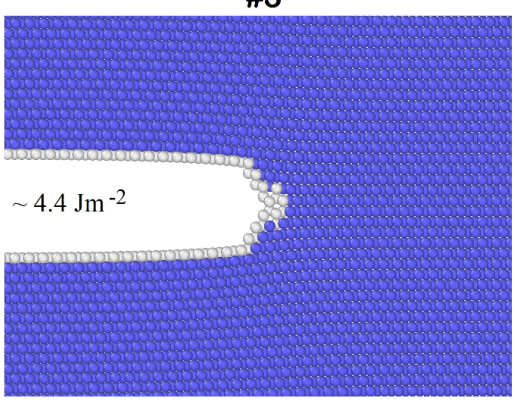

\#6

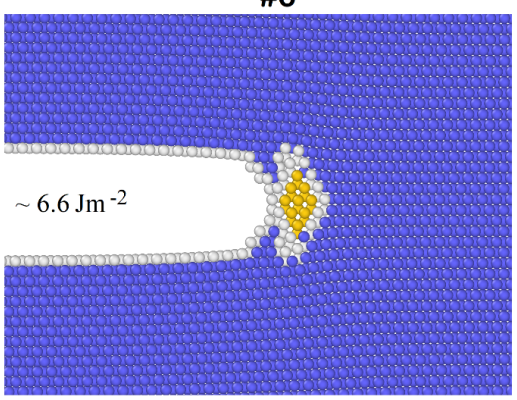

Figure 13: Snapshots of the quasi-statically loaded clean system (absent $\mathrm{H}$ ) at selected levels of applied $G_{\mathrm{I}}$. Fe atoms are coloured using a common neighbour analysis with blue indicating the BCC phase, yellow the HCP phase and white denoting an unknown coordination.

It is first instructive to examine the deformation of the clean system and understand the conditions required for $\mathrm{H}$ to be stable ahead of the crack-tip. Snapshots of the quasi-statically loaded system absent $\mathrm{H}$ at selected levels of applied $G_{\mathrm{I}}$ are included in Fig. 13. No visible crystallographic changes are seen below $G_{\mathrm{I}} \approx 4.4 \mathrm{~J} \mathrm{~m}^{-2}$ when a BCC to HCP phase transformation initiates ahead of the crack-tip (the atoms are coloured based on the local phase using a common neighbour analysis). With continued loading, the phase-transformed region 
grows $^{3}$. The BCC to HCP phase transformation is consistent with the predicted tensile response of the unit cell discussed in Appendix B and provides the mechanism to allow a hydride to form at the crack-tip. Specifically, the HCP transformation creates a more open lattice structure which is expected to increase the affinity of $\mathrm{H}$ atoms in the transformed zone compared to the BCC lattice and allow the formation of the hydrides- the effect of phase transformation is neglected in the expression for $\Delta H_{\text {tip }}$ used by Sofronis and McMeeking (1989).

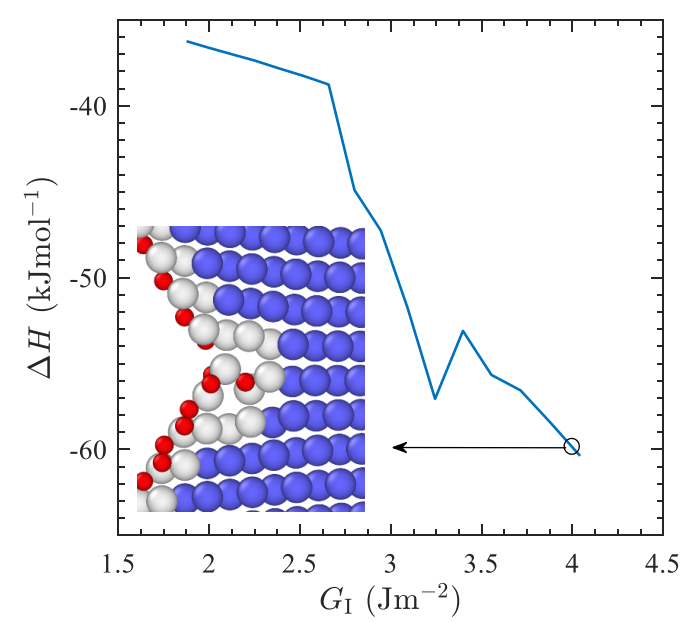

Figure 14: Predictions of the variation of the binding energy $\Delta H$ of a $H$ atom inserted in a subsurface site immediately ahead of the crack-tip (of the system of Fig. 10 with 1 ML H coverage of the crack flanks) as a function of $G_{\mathrm{I}}$. The inset shows a snapshot at load $G_{\mathrm{I}} \approx 4 \mathrm{~J} \mathrm{~m}^{-2}$ where $\mathrm{Fe}$ atoms are coloured using a common neighbour analysis with blue indicating the BCC phase and white denoting an unknown coordination while the $\mathrm{H}$ atoms (not drawn to-scale) are coloured red.

In fact, the situation is more complicated as there exists a co-operativity between stress, phase transformation and $\mathrm{H}$ in enhancing the binding affinity of $\mathrm{H}$ atoms at the crack tip. To illustrate this, we consider a system with the crack flanks fully covered with $\mathrm{H}$ and loaded to a specified $G_{\mathrm{I}}$. We then insert a $\mathrm{H}$ atom in a sub-surface site immediately ahead of the crack-tip and relax the system to calculate the binding energy of $\Delta H$ of that inserted $\mathrm{H}$ atom. This binding energy is calculated using (4.2) but with $E_{\text {ref }}$ now denoting the energy of the system with the inserted atom and $E_{i}$ the relaxed energy of the system absent this inserted $\mathrm{H}$ atom. The predicted dependence of $\Delta H$ on $G_{\mathrm{I}}$ is plotted in Fig. 14 where we also show as an inset a snapshot of the system loaded to $G_{\mathrm{I}} \approx 4 \mathrm{~J} \mathrm{~m}^{-2}$ with the inserted $\mathrm{H}$ atom directly ahead of the crack-tip. The rapid reduction in $\Delta H$ commences at $G_{\mathrm{I}} \approx 3 \mathrm{~J} \mathrm{~m}^{-2}$ even though the BCC to HCP phase transformation is seen to initiate in the absence of $\mathrm{H}$ only for $G_{\mathrm{I}}>4.4 \mathrm{~J} \mathrm{~m}^{-2}$ (Fig. 13). Comparing the $G_{\mathrm{I}} \approx 4 \mathrm{Jm}^{-2}$ snapshot in Figs. 13 and the inset of Fig. 14, it is evident that inserting an $\mathrm{H}$ atom opens up space within the Fe lattice, i.e. the $\mathrm{H}$ promotes a phase transformation analogous to the BCC to HCP phase transformation seen in Fig. 13 even for loads at which no transformation occurs in the clean system. It is this co-operativity (the phase transformation creates more space for the $\mathrm{H}$ atoms and increases their binding affinity while in turn the $\mathrm{H}$ atoms themselves promote further phase transformation) that permits the entire hydride structures shown in Figs. $12 \mathrm{~b}$ and $12 \mathrm{c}$ to be stable at loads when there is no evidence

\footnotetext{
${ }^{3}$ The MD simulations predict dislocation emission at $G_{\mathrm{I}} \approx 3 \mathrm{~J} \mathrm{~m}^{-2}$ even though such an emission event is not predicted by the quasi-static simulations at a much higher level of $G_{\mathrm{I}}$. This is because finite temperature effects in the MD simulations enable the system to overcome the barrier to dislocation emission at much lower $G_{\mathrm{I}}$ levels and the quasi-static simulations suggest that the emission barrier has not entirely vanished even at $G_{\mathrm{I}} \approx 6.6 \mathrm{~J} \mathrm{~m} \mathrm{~m}^{-2}$.
} 
of the BCC structure of the Fe lattice transforming in the clean system. However, the fact that larger hydrides are stable at higher loads is of course related to the fact that the size of the phase transformed region at the crack-tip grows with increasing load (Fig. 13).

This co-operativity between stress-induced phase transformations and $\mathrm{H}$ in the lattice is not accounted for in the Sofronis and McMeeking (1989) continuum estimate of crack-tip H concentrations. In particular, that analysis assumes a constant value of $V_{\mathrm{H}}$ given by that for an isolated $\mathrm{H}$ atom in an unstressed $\mathrm{BCC}$ lattice. The atomistic results presented here suggest that $V_{\mathrm{H}}$ is both stress and hydrogen concentration dependent with co-operativity enhancing $\left|\Delta H_{\mathrm{tip}}\right|$ compared to estimate $\Delta H_{\mathrm{tip}}=-\sigma_{\mathrm{m}} V_{\mathrm{H}}$.

\subsection{Rate of the hydride formation and speed of hydrogen-assisted cleavage crack growth}

We have demonstrated that a Fe hydride ahead of a loaded crack-tip is stable even at low levels of $c_{\mathrm{L}}$ and that this hydride promotes crack propagation by cleavage. The final key ingredient assumed in the model discussed in Section 3 is that this hydride induced cleavage results in propagation of the microcrack at a velocity $v_{\mathrm{c}}>100 \mathrm{~ms}^{-1}$ when hydrogen is supplied from within the cavity. In this section we report atomistic calculations to support this claim.

The cleavage speed of the microcrack is governed by the rate at which $\mathrm{H}$ in the crack-tip hydride can be replenished as cleavage proceeds. Here we envision the following mechanism for the entry of hydrogen from the cavity into the stressed region ahead of the crack-tip. The dissociation of hydrogen gas $\left(\mathrm{H}_{2}\right)$ into $\mathrm{H}$ and binding to available binding sites on Fe surfaces is a barrierless process. (For example, Merrill and Madix (1996) estimated the activation barrier for adsorption of $\mathrm{H}_{2}$ on $\mathrm{Fe}(100)$ to be $\approx 3 \mathrm{~kJ} \mathrm{~mol}^{-1}$ using vibrational spectroscopy measurements.) Thus, not only does the high affinity of the surface $\mathrm{H}$ sites imply that surface sites are fully occupied for any hydrogen gas pressure $p_{\mathrm{H}}$ at which embrittlement is expected but also that these sites get occupied very rapidly given a supply of $\mathrm{H}_{2}$. Thermal activation of surface $\mathrm{H}$ atoms at the crack-tip then allows them to overcome the barrier for entry into subsurface sites ahead of the crack-tip. Once within the lattice, the $\mathrm{H}$ atoms can diffuse rapidly over approximately a $1.5 \mathrm{~nm}$ region ahead of the crack-tip and form the crack-tip hydride structure. The vacant $\mathrm{H}$ sites left on the crack-tip surface by the $\mathrm{H}$ atoms that entered the system are rapidly refilled either by the fast surface diffusion of $\mathrm{H}$ atoms which is again barrierless (with vacant sites further afield from the crack-tip then refilled by the dissociation of $\mathrm{H}_{2}$ ) or the crack-tip surface sites directly replenished by dissociation of $\mathrm{H}_{2}$. This implies that the rate at which the crack-tip hydride is replenished is not limited by the availability of $\mathrm{H}_{2}$ atoms at the crack-tip. Rather the rate is set by (i) the energy barriers for $\mathrm{H}$ to enter from the surface into crack-tip subsurface sites and (ii) the energy barrier for $\mathrm{H}$ to diffuse in approximately a $1.5 \mathrm{~nm}$ region ahead of the crack-tip. Given that the $\mathrm{H}$ atoms in the crack-tip hydride need to be replenished as cleavage proceeds, we calculate these barriers for the crack loaded to $G_{\mathrm{I}} \approx$ $4 \mathrm{~J} \mathrm{~m}^{-2}$, i.e. a load level just below that required to initiate cleavage of the hydride.

The density functional theory (DFT) calculations of Jiang and Carter (2004a) predict the barrier for the entry of $\mathrm{H}$ from the BCC Fe (110) and Fe (100) unstrained surfaces into the bulk to be $\sim 96 \mathrm{~kJ} \mathrm{~mol}^{-1}$ and $37 \mathrm{~kJ} \mathrm{~mol}^{-1}$, respectively. These large barriers give the impression that ingress of $\mathrm{H}$ from the surface into the bulk is a very slow process and thus the hydride is unlikely to be restored rapidly following cleavage with $\mathrm{H}$ supplied from the cavity. However, the situation is dramatically different at a loaded crack-tip as we shall now demonstrate. Consider the hydride structure shown in the inset of Fig. 15 where we have labelled three $\mathrm{H}$ atoms $\mathrm{A}$ through $\mathrm{C}$. Atom $\mathrm{A}$ is a surface $\mathrm{H}$ atom and we first calculate the barrier for the migration of this atom to the subsurface site occupied by the atom labelled $\mathrm{B}$. The migration 
energy path was computed via the Nudged Elastic Band method (Henkelman and Jónsson, 2000) and using an initial linear path that connects the starting and final states. In this case the starting state is with atom B missing and the final with atom A missing but with all other $\mathrm{H}$ atoms in Fig. 12c present. This computed migration path energy $\mathcal{M}$ is plotted in Fig. 15 as a function of the position $\xi_{1}$ of atom A where $\xi_{1}=0$ is the current location of the crack-tip and the migrating $\mathrm{H}$ atom reaching the subsurface site originally occupied by atom $\mathrm{B}$ at $\xi_{1}=$ $0.164 \mathrm{~nm}$. Here the energy $\mathcal{M}$ is referenced to the system with all $\mathrm{H}$ atoms present, i.e. $\mathcal{M}\left(\xi_{1}\right)=E\left(\xi_{1}\right)+E_{0}-E_{\text {ref }}$, where $E\left(\xi_{1}\right)$ is the energy of the system with atom A located at $\xi_{1}$ (atom $\mathrm{B}$ absent) and $E_{\mathrm{ref}}$ the energy of the relaxed system with all $\mathrm{H}$ atoms present. The results of Fig. 15 suggest that the surface atom A needs to overcome a migration barrier $Q_{\mathrm{S}} \approx$ $9 \mathrm{~kJ} \mathrm{~mol}^{-1}$ to reach the subsurface site B. We emphasize that we do not make representations that the migration path shown here is the lowest barrier path and we can obtain multiple paths by modifying the heuristic used to initialize the path search. However, the result in Fig. 15 provides an upper bound for the surface to subsurface migration barrier and is considerably lower than the barrier computed by Jiang and Carter (2004a). Next, we consider the migration barrier for $\mathrm{H}$ to diffuse in a nm scale region ahead of the crack-tip. For illustrative purposes we choose to investigate the migration of the $\mathrm{H}$ atom labelled $\mathrm{B}$ in the inset of Fig. 15 to the location occupied by $\mathrm{H}$ atom labelled $\mathrm{C}$. The migration energy path was computed in a similar manner and shown in Fig. 15. It comprises two energy peaks with crossing over the second peak being the limiting process and therefore marked as the migration barrier with $Q_{\mathrm{H}} \approx$ $4 \mathrm{~kJ} \mathrm{~mol}^{-1}$ in Fig. 15. We note that the activation energy for diffusion of isolated $\mathrm{H}$ atoms in unstressed bulk BCC Fe is also $Q_{\mathrm{D}} \approx 4 \mathrm{~kJ} \mathrm{~mol}^{-1}$ using this EAM potential (Table B.1 in Appendix B) and thus the barrier for the internal diffusion of $\mathrm{H}$ within the hydride approximately equal to the diffusion barrier for $\mathrm{H}$ in unstressed $\mathrm{BCC}$ Fe.

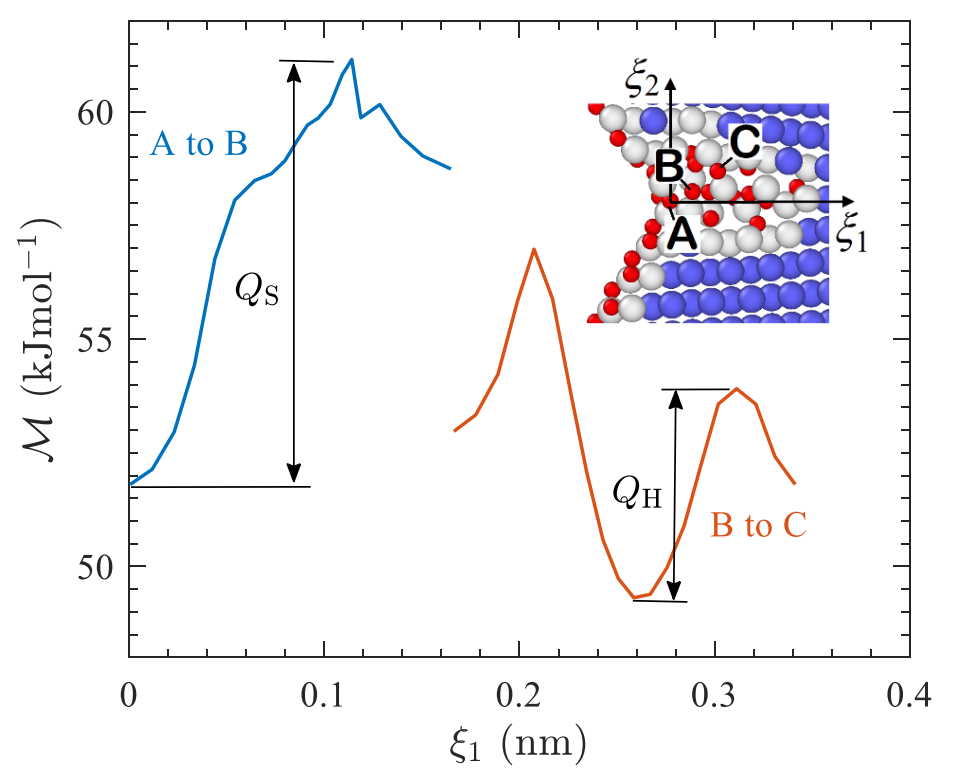

Figure 15: The migration energy $\mathcal{M}\left(\xi_{1}\right)$ paths for $\mathrm{H}$ within the hydride ahead of the crack-tip of the system loaded to $G_{\mathrm{I}}=4 \mathrm{~J} \mathrm{~m}^{-2}$. The co-ordinate defined such that $\xi_{1}=0$ is the current location of the crack-tip. Two migration paths are plotted: (i) migration of the surface $\mathrm{H}$ atom $\mathrm{A}$ to the subsurface site originally occupied by atom $\mathrm{B}$ and (ii) migration of $\mathrm{H}$ atom $\mathrm{B}$ to the site originally occupied by atom C. The critical barriers for paths (i) and (ii) are labelled $Q_{\mathrm{S}}$ and $Q_{\mathrm{H}}$, respectively. The atoms $\mathrm{A}, \mathrm{B}$ and $\mathrm{C}$ within the hydride are indicated in the inset. The colour scheme for the inset is the same as in Fig. 12. 
Given these barrier estimates we can now provide an estimate for the velocity with which the cleavage crack can propagate with hydrogen supplied from within the cavity. The barrier calculations reported above suggest that the rate of $\mathrm{H}$ ingress is limited by the barrier for $\mathrm{H}$ entry from surface sites into the subsurface. Then the rate at which $\mathrm{H}$ within the hydride is replenished (which controls the rate of propagation of the microcrack by cleavage) is set by the frequency with which the $\mathrm{H}$ atoms overcome the barrier. Each successful excursion by a $\mathrm{H}$ atom over the barrier advances the hydride by one lattice spacing of the Fe lattice. At steadystate it is reasonable to assume the microcrack cleavage velocity equals the rate of advance of the hydride and thus the microcrack velocity follows as

$$
v_{\mathrm{c}}=\omega \mathcal{A} \exp \left(-\frac{Q_{\mathrm{S}}}{R T}\right)
$$

where $\omega=10^{14} \mathrm{~Hz}$ is the atomic vibrational frequency of $\mathrm{H}$ atoms (Jiang and Carter, 2004a). With $Q_{\mathrm{S}}=9 \mathrm{~kJ} \mathrm{~mol}^{-1}$ it follows that $v_{\mathrm{c}} \approx 770 \mathrm{~ms}^{-1}$.

The calculations presented here to estimate the crack velocity are approximate as we have not performed explicit kinetic calculations of crack propagation with hydrogen being supplied from within the cavity. Nevertheless, the calculations strongly suggest fast cleavage crack propagation when hydrogen is supplied via this route. Critically recall that the cleavage velocity needs to exceed $\approx 5.4 \mathrm{~ms}^{-1}$ to inhibit blunting by dislocation emission (Fig. 2b) while a velocity of $\approx 0.05 \mathrm{~ms}^{-1}$ suffices to inhibit bulk plasticity (Fig. 7a). The barrier calculations presented here suggest hydrogen-assisted cleavage velocities well in excess of these critical values and in Section 3 we have used a conservative estimate of $v_{c}=100 \mathrm{~ms}^{-1}$ to account for any uncertainties due to the fact that detailed kinetic calculations have not been performed.

\section{Hydrogen induced reduction in tensile ductility}

The model described in Section 3 is applicable for relatively high levels of lattice hydrogen $c_{\mathrm{L}}$ such that tensile failure occurs at $\sigma_{\mathrm{f}}<\sigma_{\mathrm{Y}}$. With global plasticity then precluded, the cavity volume at the initiation of hydrogen-assisted microcrack growth is given by the elastic calculation (3.5) with $\sigma_{\text {local }}=\sigma_{\text {nuc }}$. Here we extend the model to lower levels of $c_{\mathrm{L}}$ such that tensile fracture of the specimen occurs at $\sigma_{\mathrm{f}} \geq \sigma_{\mathrm{Y}}$, i.e. global plastic deformation of the specimen precedes fracture. To do this, we need to account for the growth of the cavity due to global plastic deformation in addition to the elastic growth characterised by Eq. (3.5).

In order to calculate the contribution to the growth of the cavity from global plastic deformation, we consider a rigid-plastic solid with a strain hardening relation specified by Eq. (3.1). Strain-rate effects at this global level are neglected as tensile loading is conducted in the limit $\dot{\varepsilon}^{\infty} \rightarrow 0$. Tensile deformation nucleates and grows cavities around carbide particles of radius $R_{0}$. In the undeformed state, the carbide particles are assumed to be uniformly dispersed with a volume fraction $\eta_{0} \ll 1$ such that the carbide particle spacing $S_{0}$ is well-approximated as $S_{0}=R_{0} / \sqrt[3]{\eta_{0}}$. At the initiation of debonding of the carbide particles from the surrounding metal matrix, the volume fraction of voids in the specimen is $\eta_{0}$ and continued loading results in the growth of the radius of these voids from $R_{0}$ to $R$ and a consequent change of their volume fraction to $\eta$. With the new void spacing given by $S=R / \sqrt[3]{\eta}$, volume conservation of plastic deformation dictates

$$
R=R_{0}\left[\frac{1-\eta_{0}}{1-\eta} \frac{\eta}{\eta_{0}}\right]^{1 / 3}
$$


Then, adding the elastic straining of the void specified by the principal strains $\left(\varepsilon_{\text {local }}, \varepsilon_{\text {local }}, 0\right)$, the cavity volume including the contribution from global plastic deformation is given by

$$
\Delta V^{\mathrm{p}}=\frac{4 \pi}{3} R_{0}^{3}\left[\left(\frac{1-\eta_{0}}{1-\eta} \frac{\eta}{\eta_{0}}\right)\left(1+\varepsilon_{\text {local }}\right)^{2}-1\right] .
$$

Hydrogen-assisted crack growth initiates from the cavity surface at $\varepsilon_{\text {local }}=\varepsilon_{\text {nuc }}=$ $\sigma_{\text {nuc }}\left(1-v-2 v^{2}\right) / E$ and we label the cavity volume at this stage as $\Delta V_{\text {nuc }}^{\mathrm{p}}$. The problem is now reduced to determining $\eta$ in terms of applied tensile remote stress $\sigma^{\infty}$ which is a classical problem in the ductile growth of voids in metals (see Appendix $C$ for details). In contrast to the elastic case, the void volume fraction $\eta$ and consequently the cavity volume $\Delta V_{\text {nuc }}^{\mathrm{p}}$ are now dependent on $\sigma^{\infty}$ and we are unable to derive an explicit expression for the fracture stress $\sigma_{\mathrm{f}}$ in terms of $p_{\mathrm{H}}$. Rather, the solution involves solving an implicit set of equations as described below.

The aim here is to determine the tensile stress versus strain response and the stress $\sigma_{\mathrm{f}}<\sigma^{\text {UTS }}$ at which the specimen fails by hydrogen-assisted fracture. Consider a tensile test in a hydrogen environment with low $p_{\mathrm{H}}$ (or equivalently low $c_{\mathrm{L}}$ ). Prior to fracture, the tensile stress $\sigma^{\infty}$ versus $\varepsilon^{\mathrm{p}}$ response is given by coupled solutions of Eqs. (C.3)-(C.5) and we wish to calculate the fracture strength $\sigma_{\mathrm{f}}$. At every stage of the tensile loading (i.e. given a macroscopic tensile stress $\sigma^{\infty}$ and the corresponding macroscopic plastic strain $\varepsilon_{22}^{\mathrm{p}}$ ) we envisage hydrogen-assisted microcrack growth occurring around a carbide particle at some location within the specimen where $\sigma_{\text {local }}=\sigma_{\text {nuc }}$ due to local stress concentrations as discussed in Section 3.3. The cavity volume $\Delta V_{\text {nuc }}^{\mathrm{p}}$ around this carbide is given by Eq. (5.2) with $\eta$ obtained by the solution of Eqs. (C.3)-(C.5) for the given remote applied stress $\sigma^{\infty}$. A microcrack extends a distance $a_{\mathrm{s}}$ from the cavity surface with $a_{\mathrm{s}}$ obtained from (3.8) by replacing $\Delta V_{\text {nuc }}$ with $\Delta V_{\text {nuc }}^{\mathrm{p}}$, i.e. we obtain $a_{\mathrm{s}}\left(\sigma^{\infty}\right)$. It now remains to determine whether this microcrack extension $a_{\mathrm{s}}$ is sufficient to cause macroscopic fracture at a remote stress $\sigma^{\infty}$.

The fracture strength $\sigma_{\mathrm{f}}$ for a given $a_{\mathrm{s}}$ is given by the relation in Fig. 7b which extends to when $\sigma_{\mathrm{f}} \geq \sigma_{\mathrm{Y}}$, i.e. global plasticity has preceded fracture. The remarkable finding in Fig. $7 \mathrm{~b}$ is that in spite of global plastic deformation, the toughness remains approximately the same as in the elastic failure regime when $\sigma_{\mathrm{f}}<\sigma_{\mathrm{Y}}$. This is because while global plasticity does occur prior to the onset of crack propagation, hydrogen-assisted microcrack growth induces very high strainrates at the crack-tip and crack propagation occurs with little additional plasticity. It then follows from the results of Fig. $7 \mathrm{~b}$ that specimen failure by brittle crack propagation is only anticipated if $\sigma^{\infty} \geq \sigma_{\mathrm{f}}\left(a_{\mathrm{s}}\right)$. If this inequality is not satisfied, the hydrogen-assisted microcrack will arrest and contribute to the observed microcracking in specimens loaded in a hydrogen environment as discussed in Section 3.5. Thus, in numerical terms we continue increasing the applied $\sigma^{\infty}$ and solving the above set of equations at every stage of the loading until the equality $\sigma^{\infty}=\sigma_{\mathrm{f}}\left(a_{\mathrm{s}}\right)$ is satisfied- this value of the applied stress is then the fracture strength $\sigma_{\mathrm{f}}$ for the given $c_{\mathrm{L}}$ and the corresponding value of the plastic strain $\varepsilon_{22}^{\mathrm{p}}$ is the tensile ductility $\varepsilon_{\mathrm{f}}^{\mathrm{p}}$.

\subsection{Hydrogen induced fracture over a wide range of lattice hydrogen concentrations}

The only additional parameter introduced in the plastic void growth model is $\eta_{0}$. Novak et al. (2010) estimated that the 4340 steel they investigated had a carbide particle density of $N_{\mathrm{C}}=$ $5.5 \times 10^{11} \mathrm{~m}^{-3}$ and in all calculations reported subsequently we assume, consistent with our analysis in Section 3, that the carbide particles are of radius $R_{0}=0.15 \mu \mathrm{m}$ and therefore set $\eta_{0}=(4 / 3) \pi R_{0}^{3} N_{\mathrm{C}}=8 \times 10^{-9}$. Predictions of the tensile stress $\sigma^{\infty}$ versus strain $\varepsilon^{\infty}$ responses for lattice hydrogen concentrations in the range $0 \leq c_{\mathrm{L}} \leq 1 \mathrm{appm}$ are included in 
Fig. 8b. Tensile fracture accompanied by global plasticity occurs for hydrogen charging conditions with $c_{\mathrm{L}}<0.07$ appm and within this regime the tensile ductility (and consequently the fracture strength) increase with decreasing $c_{\mathrm{L}}$. The hydrogen-assisted fracture strength is predicted to exceed the ultimate tensile strength $\sigma^{\mathrm{UTS}}$ for $c_{\mathrm{L}}<0.013 \mathrm{appm}$ and in that regime we conclude that tensile necking intervenes to set failure with no effect of hydrogen embrittlement on the macroscopic tensile response.
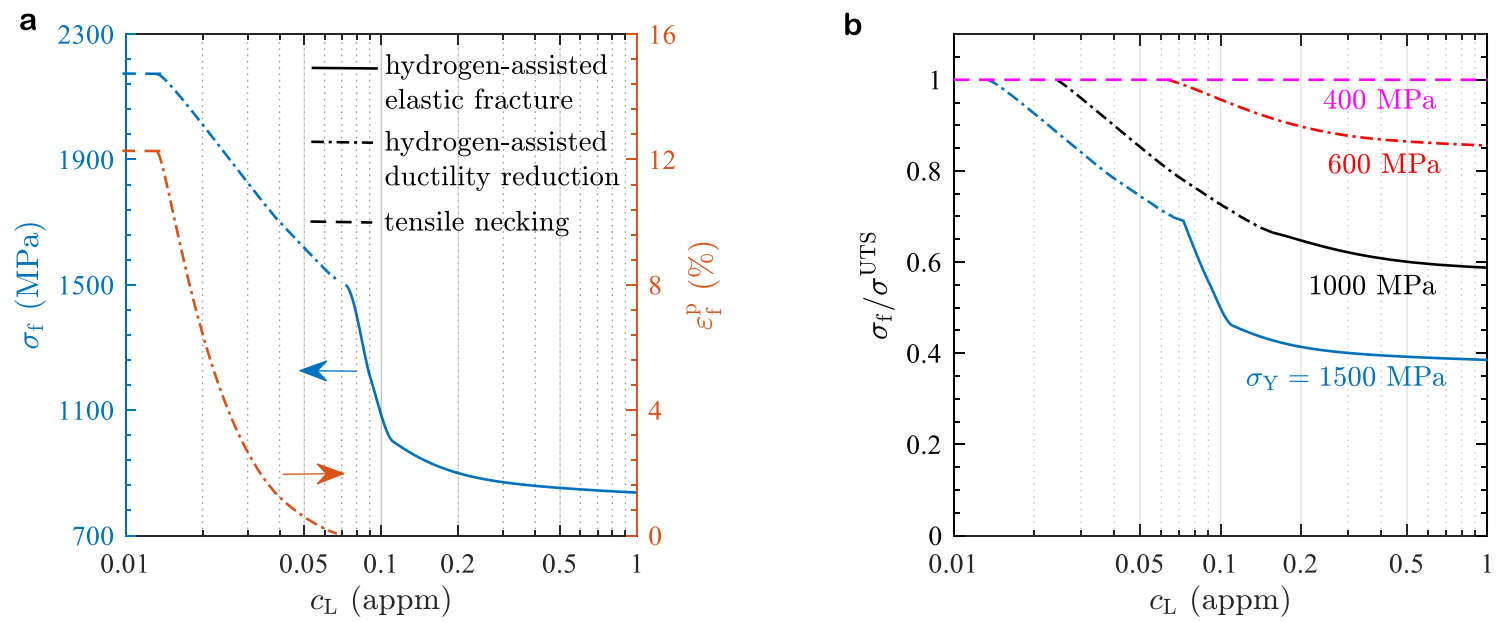

Figure 16: (a) The failure strength $\sigma_{\mathrm{f}}$ (left $y$-axis) and corresponding tensile ductility $\varepsilon_{\mathrm{f}}^{\mathrm{p}}$ (right $y$-axis) as a function of the lattice hydrogen concentration $c_{\mathrm{L}}$ for the 4340 steel with yield strength $\sigma_{\mathrm{Y}}=1500 \mathrm{MPa}$. (b) Predictions of the hydrogen environment embrittlement (HEE) index defined as the ratio of the failure strength $\sigma_{\mathrm{f}}$ in the hydrogen environment to the strength $\sigma^{\mathrm{UTS}}$ in air as a function of the lattice hydrogen concentration $c_{\mathrm{L}}$ for materials with selected values of yield strength $\sigma_{\mathrm{Y}}$. All other properties kept fixed at the values listed in Table 1 .

The predictions of the fracture strength $\sigma_{\mathrm{f}}$ and corresponding plastic failure strain $\varepsilon_{\mathrm{f}}^{\mathrm{p}}$ are summarized in Fig. 16a for a wide range of $c_{\mathrm{L}}$ in the range $0 \leq c_{\mathrm{L}} \leq 1 \mathrm{appm}$. Over this wide range of lattice hydrogen concentrations, the tensile fracture strength is predicted to drop from $\sigma_{\mathrm{f}} \approx 2200 \mathrm{MPa}$ for $c_{\mathrm{L}}<0.013 \mathrm{appm}$ when no hydrogen embrittlement is predicted to about $\sigma_{\mathrm{f}} \approx 850 \mathrm{MPa}$ at $c_{\mathrm{L}}=1 \mathrm{appm}$. The very large range of $c_{\mathrm{L}}$ values in Fig. 16a help illustrate that three regimes of failure emerge from the calculations and these are marked in Fig. 16a: (i) for $c_{\mathrm{L}}>0.07$ appm failure occurs at $\sigma_{\mathrm{f}}<\sigma_{\mathrm{Y}}$ and this is labelled hydrogen-assisted elastic fracture; (ii) for $0.013 \mathrm{appm}<c_{\mathrm{L}} \leq 0.07 \mathrm{appm}$ hydrogen-assisted fracture is accompanied by global plastic and this is labelled as hydrogen-assisted ductility reduction and (iii) for $c_{\mathrm{L}} \leq$ $0.013 \mathrm{appm}$ plastic necking is the governing failure mode with hydrogen not having any significant effect on the tensile response. We emphasize that for 4340 steel, hydrogen embrittlement as manifested by a ductility reduction occurs only for $c_{\mathrm{L}} \leq 0.07 \mathrm{appm}$. These very low values of lattice hydrogen concentration are rarely achievable in a controlled manner and thus this regime is not typically reported in experimental measurements on 4340 steels.

\subsection{Effect of yield strength}

Among the parameters that determine the susceptibility to hydrogen embrittlement, the yield strength has been identified as having a significant effect with high strength metals most susceptible irrespective of composition or structure. For example, data presented by Brahimi et al. (2017) suggests that for a given set of hydrogen charging conditions, the HEE index reduces from 0.5 for a steel with a Rockwell hardness 40 HRC to a HEE of less than 0.25 for a 52 HRC steel. We now investigate the effect of the yield strength on the tensile fracture 
strength using the model developed here. To do this in a systematic way and precisely understand the effect of yield strength we keep all parameters fixed at their values for 4340 steel (and listed in Table 1 ) and only vary $\sigma_{\mathrm{Y}}$.

The yield strength of steels (and in fact almost all metals) is typically varied by alloying and/or cold-work. These processes do not affect the theoretical strength $T_{\max }$ and thus we performed additional FE simulations to determine the dependence of $\sigma_{\mathrm{f}}$ on the extension $a_{\mathrm{s}}$ of the hydrogen-assisted microcrack for a microcrack velocity $v_{\mathrm{c}}=100 \mathrm{~ms}^{-1}$. These predictions are included in Fig. $7 \mathrm{~b}$ for $\sigma_{\mathrm{Y}}=1000 \mathrm{MPa}, 600 \mathrm{MPa}$ and $400 \mathrm{MPa}$ in addition to our reference 4340 steel case with $\sigma_{\mathrm{Y}}=1500 \mathrm{MPa}$. Two critical features emerge from these calculations: (i) failure due to crack propagation is not predicted for the $\sigma_{\mathrm{Y}}=400 \mathrm{MPa}$ case over the range of $a_{\mathrm{s}}$ values investigated here and (ii) for large values of $a_{\mathrm{s}}$, failure by crack propagation occurs at a higher value of $\sigma_{\mathrm{f}}$ for the lower yield strength materials (of course necking failure dominates at low values of $a_{\mathrm{s}}$ and this necking strength decreases with decreasing $\sigma_{\mathrm{Y}}$ ). Both these predictions are understood in terms of the cohesive zone fracture analysis presented by Tvergaard and Hutchinson (1992). With increasing $T_{\max } / \sigma_{\mathrm{Y}}$, plastic deformation around the crack-tip is enhanced and this increases the toughness of the material and thereby $\sigma_{\mathrm{f}}$ for a given value of $a_{\mathrm{s}}$ when failure is by crack propagation. In fact, for a sufficiently high $T_{\max } / \sigma_{\mathrm{Y}}$ the stresses required to open the crack cannot be generated by the material and this precludes failure by crack propagation. This is the basic finding of the analysis of Tvergaard and Hutchinson (1992) that neglected material strain-rate effects. While the strain-rate effects included in our calculations mean that our predictions quantitatively differ from those of Tvergaard and Hutchinson (1992), a number of qualitative features such as the fact that crack growth is inhibited with increasing $T_{\max } / \sigma_{\mathrm{Y}}$ remain unaffected.

These predictions of the failure strength $\sigma_{\mathrm{f}}$ as a function of $a_{\mathrm{s}}$ can be transformed to predictions of the $\sigma_{\mathrm{f}}$ as a function of the lattice hydrogen concentration $c_{\mathrm{L}}$ using the prescription detailed above. The results are summarised in Fig. 16b for the four values of $\sigma_{\mathrm{Y}}$ reported in Fig. $7 \mathrm{~b}$ in terms of the HEE index, viz. $\sigma_{\mathrm{f}} / \sigma^{\mathrm{UTS}}$. Hydrogen embrittlement is precluded in the $\sigma_{\mathrm{Y}}=$ $400 \mathrm{MPa}$ strength case with $\sigma_{\mathrm{f}} / \sigma^{\mathrm{UTS}}=1$ over the whole range of $c_{\mathrm{L}}$ values investigated here. With increasing $\sigma_{\mathrm{Y}}$ the HEE index decreases and in fact for a given $c_{\mathrm{L}}$ not only does $\sigma_{\mathrm{f}} / \sigma^{\mathrm{UTS}}$ decrease with increasing $\sigma_{\mathrm{Y}}$ but hydrogen embrittlement also persists to lower values of $c_{\mathrm{L}}$. These results are rationalized by noting that the toughness of the material decreases with decreasing $T_{\max } / \sigma_{\mathrm{Y}}$ and this implies that for a given $a_{\mathrm{s}}$, the high $\sigma_{\mathrm{Y}}$ materials have a lower fracture strength. Our findings rationalise observations that hydrogen embrittlement is mainly a phenomenon associated with high strength steels.

\section{Fracture toughness measurements; why no fast-fracture?}

The hydrogen-assisted tensile fracture mechanism proposed here is similar to that of Song and Curtin (2013) and can be broadly classified as a HEDE mechanism. However, it differs in three crucial aspects:

(i) The failure process involves fast crack propagation fuelled initially by hydrogen supplied directly by gaseous hydrogen within an internal cavity rather than by diffusion from within the bulk. This fast crack growth is then maintained, even in the absence of hydrogen, by the fact that the strain-rate sensitivity of the steel limits plasticity around the tip of the fast-growing crack. The fast propagation of the crack, in both the hydrogen-assisted and subsequent regime, is essential to maintain a sharp crack: slow hydrogen-assisted crack growth with hydrogen supplied from the 
bulk will inevitably result in crack blunting via either dislocation emission and/or bulk plasticity.

(ii) This fast-crack growth mechanism for the tensile fracture strength $\sigma_{\mathrm{f}}$, implies that $\sigma_{\mathrm{f}}$ is relatively decorrelated from the quasi-static fracture toughness measured in typical toughness tests under the same hydrogen environmental conditions.

(iii) The reduction in $\sigma_{\mathrm{f}}$ with increasing lattice hydrogen is not because of an increasing hydrogen concentration around the crack tip but rather because more hydrogen is stored in the cavities with increasing $c_{\mathrm{L}}$. This higher mass of hydrogen in the cavities results in more hydrogen-assisted fast crack growth and consequently a lower $\sigma_{\mathrm{f}}$.

Thus, unlike what is usually assumed in the HEDE explanation of tensile fracture, the model proposes that tensile fracture cannot be directly related to the static hydrogen-mediated toughness $J_{\mathrm{TH}}$ measured in identical hydrogen environments. This however leaves the question as to why the fast crack-growth fracture mechanism, which is proposed to operate in a tensile test, is not observed in toughness measurements reported in the literature.

Typical toughness tests conducted on polycrystalline alloys such as 4340 steel are based on the ASTM E1820 procedure. The specimens used in these tests have a macroscopic crack with a crack front that spans across a large number of grains in a variety of orientations as sketched in Fig. 17. The effect of hydrogen on toughness is measured by either pre-charging the specimens so as to achieve a specified level of lattice hydrogen $c_{\mathrm{L}}$ or conducted in a hydrogen environment (e.g. with gaseous hydrogen pressure $p_{\mathrm{H}}$ ). We focus on the very slow loading limit where equilibrium conditions are maintained (and the embrittlement effect is a maximum) and in such circumstances no appreciable differences are observed between the two charging conditions. Thus, in keeping with the above discussion we shall base our following discussion on a toughness test conducted in an environment with a gaseous hydrogen pressure $p_{\mathrm{H}}$.
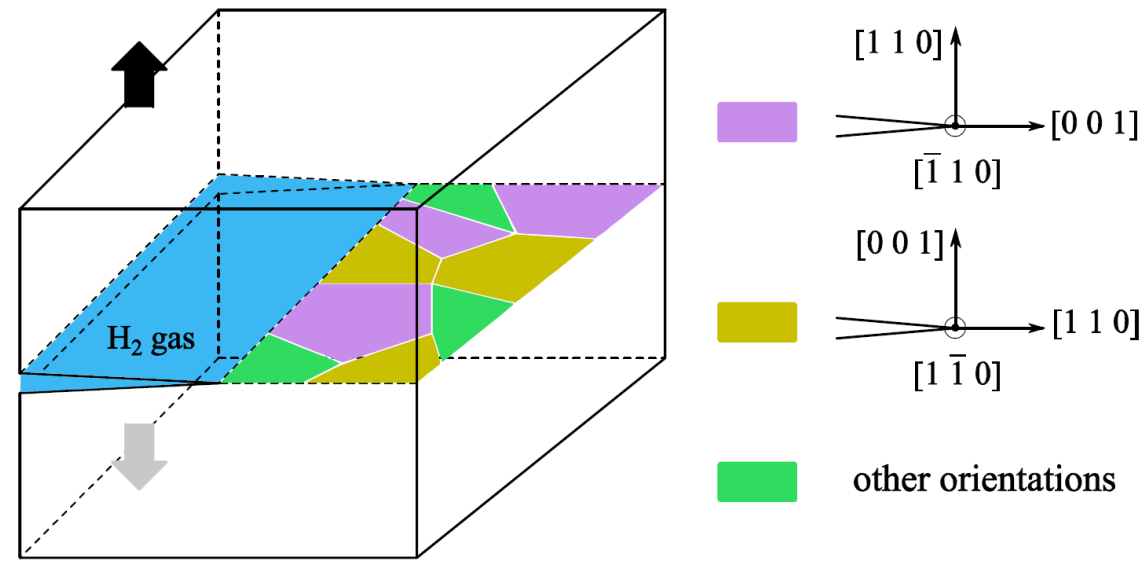

other orientations

Figure 17: Three-dimensional sketch of a typical plane strain fracture toughness test with a macroscopic crack in a polycrystalline solid. The test is shown to be conducted in a $\mathrm{H}_{2}$ atmosphere so that hydrogen gas is present between the crack flanks. A range of grain orientations exist along the initially straight crack front.

With a gaseous hydrogen pressure $p_{\mathrm{H}}$ maintained behind the crack front as sketched in Fig. 17, the scenario is very similar (although at a much larger length scale) to the nanoscopic crack emanating from cavities inside the material (Fig. 3c). The question then of course remains as to why fast-fracture is not induced by hydrogen in this macroscopic toughness test. The answer lies in the fact that in a macroscopic toughness test, multiple grain orientations exist along the crack front (Fig. 17). Some orientations where for example the crack plane lies along the (110) 
plane are prone to embrittlement and tend to cleave as discussed in detail above. However, we shall subsequently show that hydrogen and crack-tip hydrides do not embrittle and promote cleavage for cracks lying in some other crystallographic planes. This implies that the macroscopic crack front in the polycrystalline material, while expected to advance by cleavage in some locations, will be pinned back in other locations. This enhances the overall measured toughness and prevents a fast propagating crack front. On the other hand, the micron-sized internal flaw from which failure initiates in a tensile test, lies completely within a single grain and in one of the brittle orientations of the BCC Fe lattice. This permits a fast-growing hydrogen-assisted cleavage microcrack to develop. The subsequent stage-B macroscopic growth, which continues in the absence of hydrogen and through multiple differently oriented grains, occurs because of the high strain-rates at the crack-tip originally generated by the hydrogen-assisted crack growth. Here we substantiate this argument by now presenting atomistic calculations for a crack orientation that does not display a hydrogen embrittlement effect.

\subsection{Effect of hydrogen on cracks in the (001) plane}

Consider the cracked system as in Fig. 10 but now with the crack plane normal (001) aligned along the $x_{2}$-direction and periodicity enforced along the [110] crack front direction that is aligned with the $x_{3}$-axis. Both MD and quasi-static calculations were performed in a manner analogous to those described in Section 4 with mode-I loading imposed by applying a tensile strain $e_{22}$ to the system (again symmetry of the BCC lattice in this orientation implies that such an imposed strain results in pure mode-I loading). We report results in terms of the applied $G_{\mathrm{I}}=\Phi\left(e_{22}\right) L_{\text {eff }}$, where $\Phi$ now is the strain energy density of the BCC Fe lattice in this orientation (i.e. the (001) plane lies in the $x_{1}-x_{3}$ plane).

Snapshots from a MD simulation at $T=293 \mathrm{~K}$ for the system with $1 \mathrm{ML} \mathrm{H}$ coverage on the crack flanks are included in Fig. 18a at selected levels of applied $G_{\mathrm{I}}$. The atoms are coloured based on an estimated phase using a common neighbour analysis and now show a BCC to FCC phase transformation ahead of the crack-tip that initiates at $G_{\mathrm{I}} \approx 4.5 \mathrm{~J} \mathrm{~m}^{-2}$. However, unlike in the orientation discussed in Section 4 there is no dislocation emission but rather this phase transformed region grows into what resembles a twin. The associated plastic deformation results in some blunting of the crack-tip as shown in Fig. 18 a for loading to $G_{\mathrm{I}} \approx 4.8 \mathrm{~J} \mathrm{~m}^{-2}$.

Now consider the case of a hydride forming at the crack-tip. Similar to the HCP phase in the (110) orientation that opened the lattice and enhanced the affinity for $\mathrm{H}$, here the FCC phase plays a similar role. We thus loaded the system quasi-statically to $G_{\mathrm{I}} \approx 4.8 \mathrm{~J} \mathrm{~m}^{-2}$ and inserted $\mathrm{H}$ atoms in the phase transformed region and also covered the crack flanks with $\mathrm{H}$. This system was relaxed and a MD simulation $(T=293 \mathrm{~K})$ run at fixed load for 1 ps to locally equilibrate the $\mathrm{H}$ atoms. A snapshot of the crack-tip hydride at the end of this short MD run is shown in Fig. $18 \mathrm{~b}$ with the binding energies $\Delta H$ of the individual $\mathrm{H}$ atoms in the hydride marked in the snapshot. All the $\mathrm{H}$ atoms have a binding energy $\Delta H \leq-50 \mathrm{~kJ} \mathrm{~mol}^{-1}$ and the average binding energy of the entire hydride is $\langle\Delta H\rangle \approx-58 \mathrm{~kJ} \mathrm{~mol}^{-1}$. Thus, from the discussion in Section 4.2 it is clear that the hydride is thermodynamically stable at $T=293 \mathrm{~K}$ for all levels of $c_{\mathrm{L}}$ at which embrittlement is observed.

Loading of this system with the crack-tip hydride was continued via MD simulations at $T=$ $293 \mathrm{~K}$ and snapshots of the system at selected levels of $G_{\mathrm{I}} \geq 4.8 \mathrm{~J} \mathrm{~m}^{-2}$ are included in Fig. $18 \mathrm{c}$. The presence of a hydride at the crack-tip here is seen to not inhibit the crack-tip twinning. Moreover, the hydride in this orientation does not promote cleavage up to $G_{\mathrm{I}}=5.6 \mathrm{~J} \mathrm{~m}^{-2}$ computed here. This is related to the fact that the phase transformed region is located a bit away 
from the crack-tip and the hydride forms in this transformed region. Moreover, with increasing load the transformed region grows away from the crack-tip with the $\mathrm{H}$ atoms migrating further away from the crack-tip and thus inhibiting their ability to promote cleavage. These results clearly demonstrate while a crack-tip hydride might initially form in this orientation (crack plane (001) with a [1 $\overline{1} 0]$ crack front direction), the system is immune to hydrogen induced cleavage at least over the range of $G_{\mathrm{I}}$ values considered here. We note in passing that it is intriguing that the (001) orientation which is considered brittle in the absence of $\mathrm{H}$ (as it does not blunt by dislocation emission), is ductile in the context of hydrogen embrittlement.

a

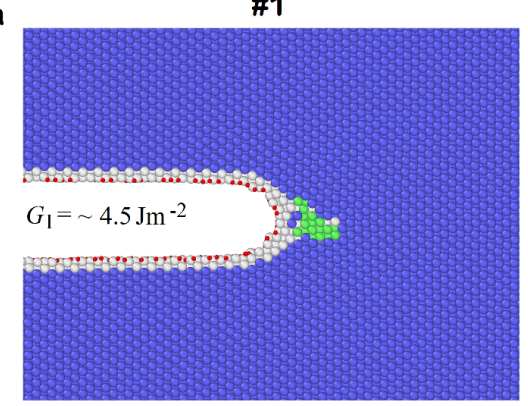

b

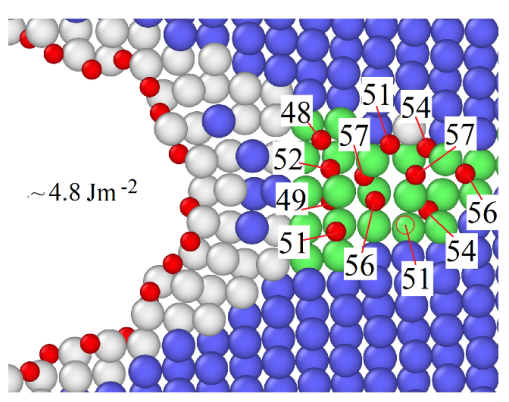

\#1

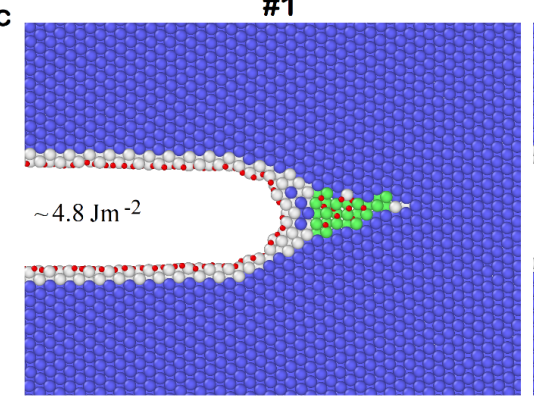

\#2

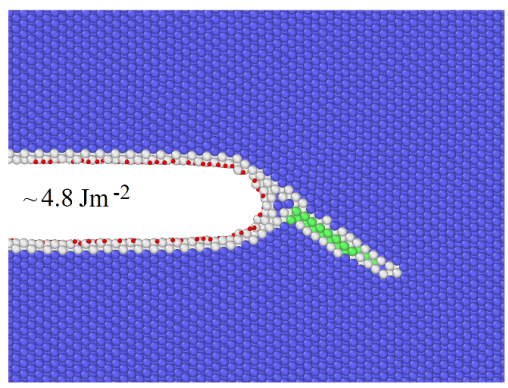

$\langle\Delta \mathrm{H}\rangle \approx-58 \mathrm{~kJ} \mathrm{~mol}^{-1}$
\#2

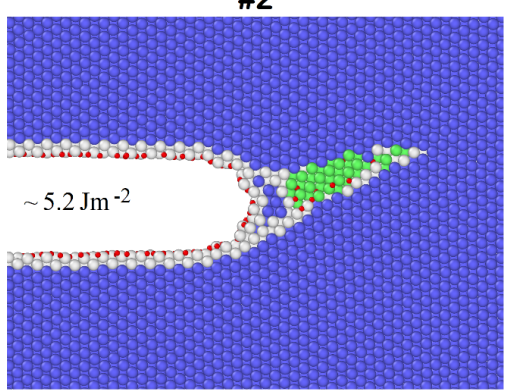

\#3

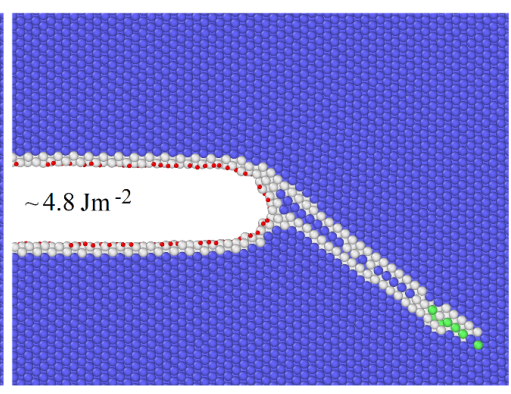

\#3

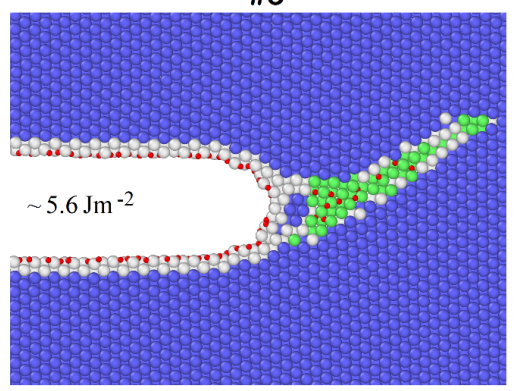

Figure 18: Atomistic predictions for loading of a crack on the Fe (001) plane with the crack front in the direction [11̄0]. (a) Snapshots from MD simulations $(T=293 \mathrm{~K})$ at selected levels of applied $G_{\mathrm{I}}$ for (a) a system with $1 \mathrm{ML} \mathrm{H}$ coverage on the crack flanks and (c) a system with $1 \mathrm{ML} H$ coverage on the crack flanks and a hydride ahead of the crack-tip. (b) The binding energies of $\mathrm{H}$ atoms in the hydride structure ahead of the crack-tip for the system loaded to $G_{\mathrm{I}} \approx 4.8 \mathrm{Jm}^{-2}$ (the values shown are $-\Delta H$ in units of $\mathrm{kJ} \mathrm{mol}^{-1}$ ). The average binding energy $\langle\Delta H\rangle$ of the entire hydride is also indicated. Fe atoms are coloured using a common neighbour analysis with blue indicating the BCC phase, green the FCC phase and white denoting an unknown coordination. The $\mathrm{H}$ atoms (not drawn to-scale) are coloured red (one of them is shown in (b) as an open red circle as it sits behind the Fe atoms).

Based on these results we conclude that the combination of grain orientations along the crack front, some of which that are prone to hydrogen-assisted cleavage and some that remain relatively ductile, results in the measured $J_{\mathrm{TH}}$ in a toughness test in a hydrogen environment being lower than the $J_{\text {IC }}$ in the absence of hydrogen. Fracture in such a toughness test is therefore expected to be accompanied by significant crack-front plasticity where the role of 
plasticity size effects such as those considered by Martínez-Pañeda et al. (2016) may play a role. However, this $J_{\mathrm{TH}}$ is still considerably higher than the effective toughness of the material in a uniaxial tension test in an identical hydrogen environment.

\subsection{Suggestion for some critical experiments}

The model proposed here has been shown to explain a large number of phenomena/features observed in relation to the effect of hydrogen on the tensile response of steels such as 4340 . These include:

(i) A tensile cleavage fracture strength that does not correlate with the measured toughness in an identical hydrogen environment.

(ii) A tensile strength that scales with the lattice hydrogen concentration but is independent of the trapped hydrogen.

(iii) The extensive microcracking that usually accompanies hydrogen embrittlement as well as the fact that the fracture typically initiates from micron-sized inclusions.

(iv) The acoustic emissions measured for tensile loading in a hydrogen atmosphere are hypothesised to be associated with the dynamic formation of microcracks predicted by our atomistic simulations.

(v) The statistical nature of the fracture strength, viz. tensile strength measurements in hydrogen environments exhibit large scatter in the data.

(vi) High strength steels are significantly more prone to hydrogen embrittlement compared to their lower yield strength counterparts.

While these serve to give confidence in the validity of the model, it is however always appealing when a new model suggests the existence of phenomena that have not yet been experimentally reported - if these predictions are borne out by future experiments it serves to significantly reinforce the validity of the model. Here we propose some such critical experiments.

One key feature of the model is that we predict that hydrogen embrittles Fe for cracks only in certain orientations and that cleavage fracture will only occur if a mechanism to rapidly supply hydrogen to the crack-tip exists. This suggests the following set of toughness experiments on Fe single crystals. First consider a crack orientation where crack-tip hydrides are expected to promote cleavage. This includes, but not expected to be limited to, cracks on (111) plane (as analysed by Song and Curtin (2013)) as well as our investigation of a crack on the (110) plane. Our simulations of a crack on the (110) plane with the crack front in the [110] direction suggest:

(i) A toughness measurement carried out in a gaseous hydrogen atmosphere at pressure $p_{\mathrm{H}}$ will result in fast cleavage fracture at $K_{\mathrm{I}}=K_{\mathrm{TH}} \approx 1 \mathrm{MPa} \sqrt{\mathrm{m}}$.

(ii) On the other hand, a test where an identical specimen is pre-charged to a lattice hydrogen concentration $c_{\mathrm{L}}$ where $c_{\mathrm{L}}$ in this experiment and $p_{\mathrm{H}}$ in (i) are related through Eqs. (3.2) and (3.4) will not result in fast-fracture. Rather we predict that such a test will lead to crack-tip blunting and consequently a higher toughness. This is because the supply of $\mathrm{H}$ to the crack-tip is now via diffusion through the bulk and this slower supply route means that a hydride does not constantly persist at the crack-tip. This permits blunting of the crack-tip by dislocation emission.

We thus predict that the nature of the hydrogen-charging will have a dramatic effect on the crack growth for a Fe single crystal in this orientation. No experimental data has been reported to-date to suggest such an effect. Finally, our simulations also indicate that a Fe single crystal with a crack on the (001) plane and crack front in the [110] direction will have a considerably higher toughness. 


\section{Concluding remarks}

We have presented a combined atomistic/continuum model to rationalize a number of apparently contrasting observations for the hydrogen-assisted tensile fracture of steels. While the overall model is expected to be applicable generally to ferritic steels we have presented results for a 4340 steel: detailed material property and measurements and microstructural characterisations are available in the literature for this steel enabling detailed predictions without fitting parameters. The critical aspect of the model is that it recognises that both cracktip dislocation emission and bulk plasticity around the crack-tip need to be limited to allow cleavage fracture to occur. Here we show that this is possible in two stages. Stage-A is a hydrogen-assisted cleavage regime where hydrogen is supplied from hydrogen gas within a micron-sized cavity that forms by the debonding of an inclusion such as a carbide particle. This hydrogen supply route promotes the fast propagation of a crack emanating from the surface of the cavity in a cleavage mode. This stage ends when the hydrogen gas within the cavity is exhausted and is followed by a stage-B regime where cleavage crack growth persists in the absence of hydrogen supply given an appropriate level of remote tensile stress. This is because the high crack-tip strain-rates generated by the hydrogen-assisted growth in stage-A limit bulk plasticity. This embrittlement mechanism thus has analogies to the cleavage fracture of ferritic steels (in the absence of hydrogen) that is initiated from the sudden fracture of brittle second phase inclusions such as carbides (Hahn, 1984) and analysed by Kroon and Faleskog (2005).

Atomistic calculations, using an Embedded Atom Model (EAM) for interactions between iron and hydrogen atoms, provide support for stage-A of the process and include a number of novel findings. These are: (i) hydrides form at crack-tips due to a co-operative mechanism between a stress-induced phase transformation that increases the affinity for $\mathrm{H}$ and the $\mathrm{H}$ in turn further enhancing the phase transformation; (ii) the barrier for the entry of surface $\mathrm{H}$ into subsurface sites is greatly reduced at the loaded crack-tip and this combined with a low diffusion barrier allows for the rapid formation of a crack-tip hydride fed by hydrogen from the cavity and (iii) hydrides promote cleavage on the $\mathrm{Fe}$ (110) plane but do not prevent twinning and crack-tip blunting on the $\mathrm{Fe}(001)$ plane. Continued fast propagation in stage- $\mathrm{B}$, now in the absence of $\mathrm{H}$ supply, is supported by continuum calculations in a rate dependent elastic-plastic solid with fracture modelled using a cohesive zone. The cohesive zone has a fracture energy of $4.8 \mathrm{~J} \mathrm{~m}^{-2}$ and a peak strength of $15 \mathrm{GPa}$ both of which are representative for the atomic separation of Fe. The calculations show that these high crack opening stresses are able to be generated because strain-rates at the tip of a fast propagating cracks are sufficiently high for drag on the motion of dislocations due to phonon scattering to limit plasticity.

The calculations reported rationalize a number of observations of hydrogen embrittlement including: (i) the disconnect between the measured toughness and tensile strength of steels in identical hydrogen environments; (ii) the dependence of the level of embrittlement primarily on the lattice or diffusible hydrogen rather than trapped hydrogen; (iii) the extensive microcracking that is associated with hydrogen embrittlement; and (iv) the higher susceptibility of high strength steels to hydrogen embrittlement. Moreover, our calculations point towards some critical experiments, with some counterintuitive expected findings, that have not been reported to-date: future experiments to confirm or refute these predictions will help gauge the validity of the model developed here. It is emphasized that the model as presented here is applicable in the limit of slow loading such that hydrogen is in equilibrium (spatially uniform chemical potential) prior to the onset of hydrogen-assisted cleavage. This represents an upperbound on the level of embrittlement with it being well-established that embrittlement reduces with increasing applied strain rate. Extension of the present model to higher applied strain rates 
where we need to account for the fact that the voids may not fill with hydrogen gas to the equilibrium pressure prior to the onset of cleavage is part of on-going work by the authors.

Finally, we note that the $\mathrm{R} 6^{4}$ and equivalent procedures that are widely used to assess the integrity of structures containing defects are based on the usual notions of fracture mechanics wherein tensile strength can be related to the macroscopic toughness through a defect size. Here, we argue that the disconnect between the hydrogen-assisted toughness and tensile strength is because failure in these situations occurs via different mechanisms. The implications of this for the applicability of procedures such as R6 for the design of steel structures in hydrogen environments needs further investigation.

Appendix A: Minimum cleavage crack velocity to avoid blunting by dislocation emission For BCC Fe, the mode-I stress intensity factor $K_{\mathrm{I}}$ for dislocation emission in the absence of hydrogen is $K_{\text {emit }} \approx 1 \mathrm{MPa} \sqrt{\mathrm{m}}$ (see atomistic calculations reported in Section 4 ). On the other hand, the $K_{\mathrm{I}}$ required for cleavage follows from the work of separation $G_{0}=4.8 \mathrm{~J} \mathrm{~m}^{-2}$ for Fe again in the absence of hydrogen (Jiang and Carter, 2004b) as

$$
K_{\text {cleavage }}=\sqrt{\frac{E G_{0}}{\left(1-v^{2}\right)}} \approx 1 \mathrm{MPa} \sqrt{\mathrm{m}} .
$$

This estimate for $K_{\text {cleavage }}$ assumes the existence of a sharp crack such that an opening traction $T_{\text {max }}=15 \mathrm{GPa}$ can readily be attained immediately ahead of the crack tip (see Section 3). However, continued dislocation emission will blunt the crack preventing the attainment of this traction leading to a ductile response. Here, we show that a crack travelling at a velocity $v_{\mathrm{c}}>$ $v_{\text {blunt }}^{\text {crit }}$ will not blunt sufficiently to prevent cleavage. The analysis comprises two steps: (i) estimation of the number of dislocations $\alpha$ that need to be emitted by a stationary crack to sufficiently blunt so that the opening tractions cannot exceed $T_{\max }$ at $K_{\mathrm{I}}=K_{\text {cleavage }}$ and (ii) estimation of the rate of dislocation emission $\dot{\mathcal{N}}$. These two estimates can be combined to calculate $v_{\text {blunt }}^{\text {crit }}$.

For simplicity, we consider the plane strain case of large plate with a central notch of length $2 \lambda$ and root radius $r_{\mathrm{n}} \ll \lambda$ subjected to a remote tensile stress $\sigma^{\infty}$. Now consider the distribution of the opening stress $\sigma_{y y}(x)$ along $y=0$ ahead of the notch root where the origin of $(x, y)$ coordinate system is at the notch root. At distances $x \gg r_{\mathrm{n}}$, the stress is well-approximated by a K-field solution such that

$$
\sigma_{y y}=\frac{K_{\mathrm{I}}}{\sqrt{2 \pi x}}
$$

where $K_{\mathrm{I}}=\sigma^{\infty} \sqrt{\pi \lambda}$ while in the limit $x \rightarrow 0$, opening stress attains the value dictated by the elastic stress concentration ahead of the notch (Rice, 1968), viz.

$$
\sigma_{y y} \rightarrow \sigma_{\max }=2.43 \sigma^{\infty} \sqrt{\frac{\lambda}{r_{\mathrm{n}}}}=2.43 \frac{K_{\mathrm{I}}}{\sqrt{\pi r_{\mathrm{n}}}} .
$$

For cleavage to occur, $\sigma_{\max }$ needs to attain a value $\sigma_{\max }>T_{\max }=15 \mathrm{GPa}$ and upon setting $K_{\mathrm{I}}=K_{\text {cleavage }}=K_{\text {emit }} \approx 1 \mathrm{MPa} \sqrt{\mathrm{m}}$, we obtain the maximum root radius for cleavage as $r_{\mathrm{n}} \approx$ $8.3 \mathrm{~nm}$. Given an initially atomistically sharp crack, the crack will blunt to a radius $r_{\mathrm{n}}=$

\footnotetext{
${ }^{4}$ CEGB R6, "Assessment of the Integrity of Structures Containing Defects", Revision 4, British Energy, Gloucester, UK, 2001.
} 
$8.3 \mathrm{~nm}$ upon emitting $\alpha=2 r_{\mathrm{n}} / b \approx 66$ dislocations where $b=0.25 \mathrm{~nm}$ is the Burgers vector magnitude. We emphasize here that this estimate of $\alpha$ that defines a blunt crack holds even in the presence of a crack-tip hydride as the mode-I stress intensity factor required for cleavage of the hydride also is $K_{\text {cleavage }}^{\mathrm{H}} \approx 1 \mathrm{MPa} \sqrt{\mathrm{m}}$; see Section 4 .

Now to estimate the rate $\dot{\mathcal{N}}$ of dislocation emission recall that for a given applied remote $K_{\mathrm{I}}$, the effective crack-tip stress-intensity $K_{\text {eff }}$ reduces below $K_{\text {emit }}$ immediately after an emission event due to the elastic shielding of the crack by the emitted dislocation. The rate of emission is then limited by the time it takes for $K_{\text {eff }}$ to recover back to a level $K_{\text {emit }}$ as the dislocation moves away from the crack-tip. The effective crack-tip stress intensity $K_{\text {eff }}$ is given by the sum of the remote applied $K_{\mathrm{I}}$ and the stress intensity factor $K_{\mathrm{D}}$ at the crack-tip due to the presence of the emitted dislocation, i.e. $K_{\text {eff }}=K_{\mathrm{I}}+K_{\mathrm{D}}$. The stress intensity factor $K_{\mathrm{D}}$ due to an emitted dislocation with Burgers vector magnitude $b$ that is now at a distance $z$ from the crack-tip is given by (Majumdar and Burns, 1981)

$$
K_{\mathrm{D}} \approx-\frac{\mu b}{\sqrt{2 \pi z}}
$$

where $\mu=E /[2(1+v)]$ is the shear modulus. The crack tip will then emit the next dislocation when $K_{\text {eff }}$ recovers to $K_{\text {emit }}$ after the first dislocation has reached a distance $z_{\text {sh }}$ away from the crack-tip. This distance is obtained by setting $K_{\mathrm{eff}}=K_{\text {emit }}$ and substituting for $K_{\mathrm{D}}$ from Eq. (A.4) as

$$
z_{\mathrm{sh}}=\frac{1}{2 \pi}\left[\frac{\mu b}{K_{\mathrm{I}}-K_{\mathrm{emit}}}\right]^{2} .
$$

The continuum elastic dislocation stress fields used to compute (A.5) lose accuracy when the dislocations are within a few atomic spacing from the crack-tip. In fact, a dislocation can only be thought to have fully nucleated once it is at a distance $10 \mathrm{~b}$ from the crack-tip: another dislocation cannot be emitted until this first dislocation has at-least been fully nucleated. Hence, it is reasonable to cut-off Eq. (A.5) for $z_{\text {sh }}<10 b$ and define a critical distance as $\bar{z}_{\text {sh }} \equiv$ $\max \left(z_{\mathrm{sh}}, 10 b\right)$. The rate of emission can then be calculated from the time it takes for the emitted dislocation to reach $\bar{z}_{\mathrm{sh}}$. There is a large repulsive force (Peach-Koehler force) tending to drive the emitted dislocation away from the crack-tip and therefore we expect an emitted dislocation to be moving at a speed approaching the elastic shear wave speed $V_{\mathrm{s}}$ in the vicinity of the crack-tip. The time for the shielding effect of the emitted dislocation to diminish such that $K_{\text {eff }}$ recovers back to $K_{\text {emit }}$ is then given by $\bar{z}_{\mathrm{sh}} / V_{\mathrm{s}}$ and consequently the rate of dislocation emission is $\dot{\mathcal{N}}=V_{\mathrm{s}} / \bar{z}_{\text {sh. }}$.

Cleavage is precluded if a crack emits more than $\alpha$ dislocations and therefore cannot attain a tip stress greater than $T_{\text {max }}$ before it advances one atomic spacing $\mathcal{A}$, where $\mathcal{A}=0.286 \mathrm{~nm}$ is the lattice constant for BCC Fe. For a crack propagating at a speed $v_{\mathrm{c}}$, the number of dislocations $\mathcal{N}$ emitted in the time taken for the cleavage crack to propagate one atomic spacing is given by $\mathcal{N}=\dot{\mathcal{N}} \mathcal{A} / v_{\mathrm{c}}$. Then setting $\mathcal{N}=\alpha$ and substituting $\dot{\mathcal{N}}=V_{\mathrm{s}} / \bar{z}_{\text {sh }}$ gives the critical velocity above which dislocation emission will not result in blunting sufficient to prevent cleavage is given by

$$
v_{\text {blunt }}^{\text {crit }}=\frac{V_{\mathrm{s}} \mathcal{A}}{\alpha \bar{z}_{\mathrm{sh}}} .
$$

Predictions of $v_{\text {blunt }}^{\text {crit }}$ with $\alpha=66$ are included in Fig. $2 \mathrm{~b}$ as a function of $K_{\mathrm{I}} / K_{\text {emit }}$, where we have taken $b=0.25 \mathrm{~nm}, V_{\mathrm{s}}=\sqrt{\mu / \rho} \approx 3100 \mathrm{~ms}^{-1}\left(\rho=7850 \mathrm{~kg} \mathrm{~m}^{-3}\right.$ is the density of Fe and $\mu=76.9 \mathrm{GPa}$ ) and $K_{\text {emit }}=1 \mathrm{MPa} \sqrt{\mathrm{m}}$. 
We recognise that there are differing views in the literature on brittle crack growth due to a fast-growing crack in a nominally ductile metal. For example, the experiments of Han (1984) and others suggest that brittle fracture can ensue in Fe due to the dynamic failure of a carbide particle while the MD simulations of Rajan and Curtin (2016) suggest that even a crack travelling a high velocity will tend to blunt by dislocation emission in a ductile metal. While the precise reasons behind these differing findings needs further investigation, it is possible that the differences lie in the definition of crack-tip blunting. Typically, in MD simulations crack-tip blunting is defined as the onset of the emission of the first dislocation while here we suggest that a crack can propagate by cleavage even though it has emitted $\alpha=66$ dislocations.

Table B.1: Some relevant material property values extracted from the EAM Fe-H potential.

\begin{tabular}{|l|c|}
\hline \multicolumn{1}{|c|}{ Property } & Value \\
\hline Diffusion barrier for $\mathrm{H}$ in $\alpha-\mathrm{Fe}, Q_{\mathrm{D}}$ & $3.86 \mathrm{~kJ} \mathrm{~mol}^{-1}$ \\
\hline$\alpha-\mathrm{Fe}$ lattice constant, $\mathcal{A}$ & $0.2855 \mathrm{~nm}$ \\
\hline $\begin{array}{l}\text { Surface energy of the } \mathrm{Fe} \mathrm{(110)} \\
\text { surface }\end{array}$ & $1.617 \mathrm{~J} \mathrm{~m}^{-2}$ \\
\hline $\begin{array}{l}\text { Binding energy } \Delta H \text { of } \mathrm{H} \text { in three- } \\
\text { fold sites on Fe }(110)\end{array}$ & $-66 \mathrm{~kJ} \mathrm{~mol}^{-1}$ \\
\hline $\begin{array}{l}\text { Binding energy } \Delta H \text { of } \mathrm{H} \text { in bridge } \\
\text { site on Fe (110) }\end{array}$ & $-68 \mathrm{~kJ} \mathrm{~mol}^{-1}$ \\
\hline $\begin{array}{l}\text { Surface energy of Fe }(110) \text { with } 1 \\
\text { ML coverage of } \mathrm{H} \text { in three-fold sites }\end{array}$ & $0.726 \mathrm{~J} \mathrm{~m}^{-2}$ \\
\hline $\begin{array}{l}\text { Surface energy of Fe (110) with } 1 \\
\text { ML coverage of } \mathrm{H} \text { in bridge sites }\end{array}$ & $0.674 \mathrm{~J} \mathrm{~m}^{-2}$ \\
\hline
\end{tabular}

\section{Appendix B: Some salient properties of the Fe-H EAM potential}

With the $x_{1}, x_{2}$ and $x_{3}$-directions aligned with the [100], [010] and [001] crystallographic directions, respectively the linear elastic stress versus strain relation for the BCC Fe lattice is written in Voigt notation as $\sigma_{i}=c_{i j} \varepsilon_{j}(i, j=1,2, \ldots, 6)$, where $c_{i j}=c_{j i}$. Cubic symmetry dictates that $c_{11}=c_{22}=c_{33}, c_{44}=c_{55}=c_{66}$ and $c_{12}=c_{13}=c_{23}$ with all other $c_{i j}=0$, i.e. the elastic relation can be fully specified in terms of three constants and for the EAM potential used here they are: $c_{11}=243 \mathrm{GPa}, c_{12}=145 \mathrm{GPa}$ and $c_{44}=116 \mathrm{GPa}$. Rotating the crystal such that the $x_{1}, x_{2}$ and $x_{3}$-directions are aligned with the [001], [110] and [110] crystallographic directions, respectively (i.e. the crystal orientation in Fig. 10), the elastic relation in Voigt notation reads $\{\boldsymbol{\sigma}\}=\left[\mathbf{c}^{\prime}\right]\{\boldsymbol{\epsilon}\}$ such that

$$
\left\{\begin{array}{l}
\sigma_{1} \\
\sigma_{2} \\
\sigma_{3} \\
\sigma_{4} \\
\sigma_{5} \\
\sigma_{6}
\end{array}\right\}=\left[\begin{array}{cccccc}
c_{11}^{\prime} & c_{12}^{\prime} & c_{13}^{\prime} & 0 & 0 & 0 \\
c_{12}^{\prime} & c_{22}^{\prime} & c_{23}^{\prime} & 0 & 0 & 0 \\
c_{13}^{\prime} & c_{23}^{\prime} & c_{33}^{\prime} & 0 & 0 & 0 \\
0 & 0 & 0 & c_{44}^{\prime} & 0 & 0 \\
0 & 0 & 0 & 0 & c_{55}^{\prime} & 0 \\
0 & 0 & 0 & 0 & 0 & c_{66}^{\prime}
\end{array}\right]\left\{\begin{array}{c}
\varepsilon_{1} \\
\varepsilon_{2} \\
\varepsilon_{3} \\
\varepsilon_{4} \\
\varepsilon_{5} \\
\varepsilon_{6}
\end{array}\right\},
$$

with $c_{11}^{\prime}=243 \mathrm{GPa}, c_{22}^{\prime}=c_{33}^{\prime}=310 \mathrm{GPa}, c_{12}^{\prime}=c_{13}^{\prime}=145 \mathrm{GPa}, c_{23}^{\prime}=78 \mathrm{GPa}, c_{44}^{\prime}=$ $49 \mathrm{GPa}$ and $c_{55}^{\prime}=c_{66}^{\prime}=116 \mathrm{GPa}$. For mode-I loading as shown in Fig. 10, the stress intensity factor $K_{\mathrm{I}}$ and energy release rate $G_{\mathrm{I}}$ are related through $G_{\mathrm{I}}=C_{\mathrm{G}} K_{\mathrm{I}}^{2}$ where the factor $C_{\mathrm{G}}$ (Sih et al., 1965) is given by 


$$
C_{\mathrm{G}}=\sqrt{\frac{B_{11}^{\prime} B_{22}^{\prime}}{2}\left(\sqrt{\frac{B_{22}^{\prime}}{B_{11}^{\prime}}}+\frac{2 B_{12}^{\prime}+B_{66}^{\prime}}{2 B_{11}^{\prime}}\right)} .
$$

Here, $B_{i j}^{\prime}$ are plane-strain elastic compliances, viz.

$$
B_{i j}^{\prime}=s_{i j}^{\prime}-\frac{s_{i 3}^{\prime} s_{3 j}^{\prime}}{s_{33}^{\prime}} \quad(i, j=1,2, \ldots, 6),
$$

where $s_{i j}^{\prime}$ are components of compliance matrix $\left[\mathbf{s}^{\prime}\right] \equiv\left[\mathbf{c}^{\prime}\right]^{-1}$. Then using the values of $c_{i j}^{\prime}$ for this EAM potential we obtain $C_{\mathrm{G}}=3.87 \mathrm{~Pa}(\mathrm{MPa})^{-2}$ for the orientation in Fig. 10. A similar analysis can be performed for the orientation with the (001) crack plane discussed in Section 6 and $G_{\mathrm{I}}$ and $K_{\mathrm{I}}$ in that case are related via $C_{\mathrm{G}}=4.37 \mathrm{~Pa}(\mathrm{MPa})^{-2}$. Some further relevant properties of the Fe-H EAM potential are listed in Table B.1 for the sake of completeness.
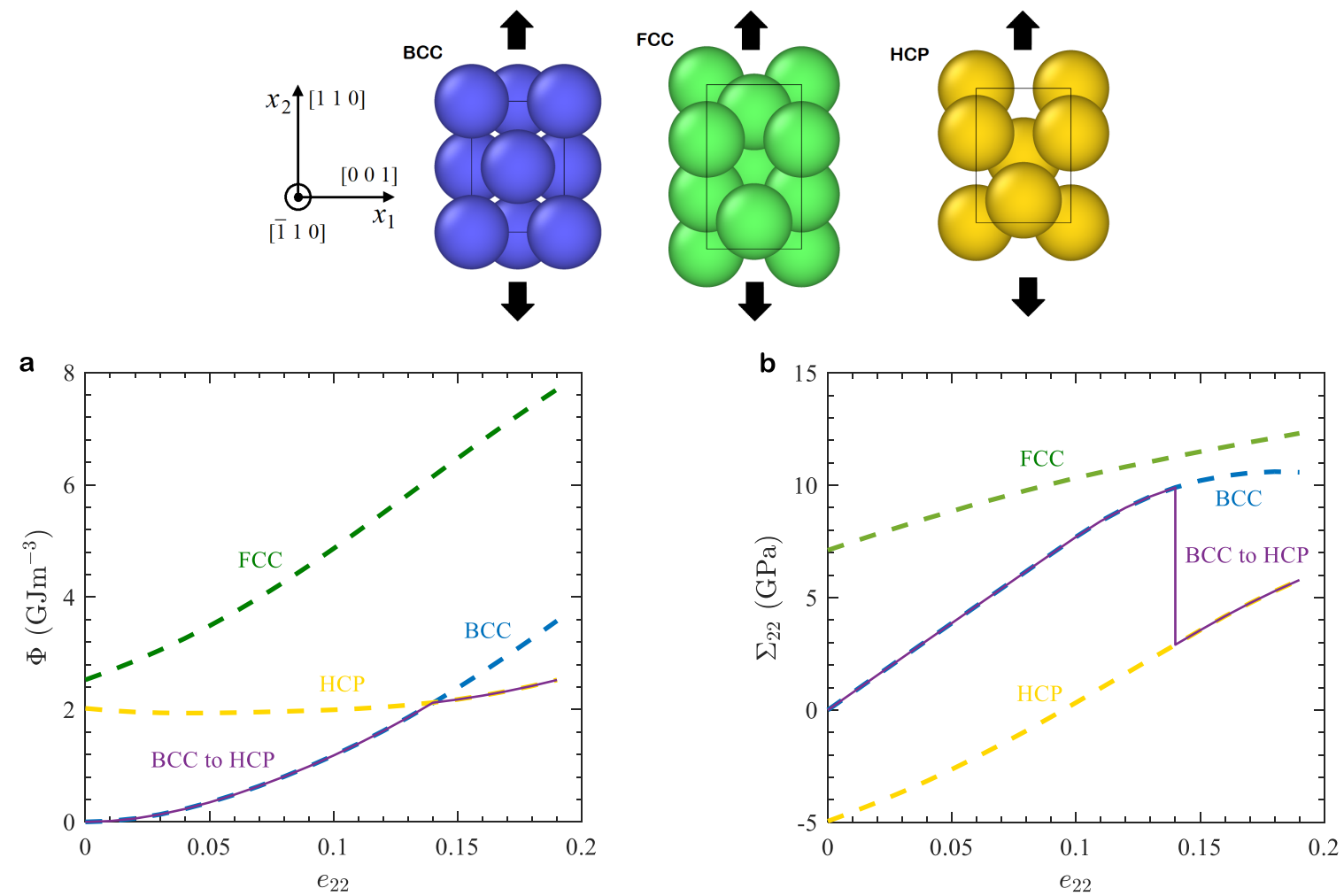

Figure B.1: (a) Predictions of the strain energy density $\Phi$ versus strain $e_{22} \equiv F_{22}-1$ for BCC, FCC and HCP Fe. (b) The corresponding nominal stress $\Sigma_{22} \equiv \partial \Phi / \partial F_{22}$ versus $e_{22}$ predictions. In both (a) and (b) we include predictions where the phase is constrained to remain unchanged as well as the case where phase transformations are permitted. In the latter, a BCC to the HCP phase transformation is predicted at $e_{22} \approx 0.14$. In both (a) and (b) the strain $e_{22}$ is defined with respect to the unstrained $\mathrm{BCC}$ unit cell with the unit cell orientated identically to the cracked geometry in Fig. 10.

It is also instructive to investigate the predicted tensile response of the Fe lattice as described by this EAM potential in order to understand the material response in the close vicinity of the crack tip. Consider the BCC Fe lattice orientated as in Fig. 10 (also see inset in Fig. B.1 that shows the BCC unit cell). We quasi-statically impose a deformation gradient $F_{i j}$, that is representative of the strain-state directly ahead of the crack-tip, on a unit cell of the lattice. Specifically, we impose $F_{i j}=0$ for $i \neq j, F_{11}=1, F_{22}=e_{22}+1$ where $e_{22}$ is the nominal strain in the $x_{2}-$ direction and enforce periodicity in the $x_{3}-$ direction with $F_{33}=1$. First consider the case where all atoms of the unit cell are constrained to displace affinely consistent 
with the imposed $F_{i j}$. The system is then constrained to remain in its BCC phase and the corresponding predictions of the strain energy per unit volume $\Phi$ and work-conjugate nominal stress $\Sigma_{22} \equiv \partial \Phi / \partial F_{22}$ are included in Figs. B.1a and B.1b, respectively, as a function of $e_{22}$. Next, we again impose $F_{i j}$ on the unit cell but now relax the positions of the internal Fe atoms at each stage of the loading to allow the system to attain its lowest energy state. The corresponding predictions of $\Phi$ and $\Sigma_{22}$ are also included in Fig. B.1. The two simulation schemes give identical predictions for $e_{22}<0.14$ but the simulation where we permit the system to relax predicts a phase transformation and an associated stress drop at $e_{22} \approx 0.14$ with the BCC phase transforming to the HCP phase.

For the sake of completeness, we also illustrate the responses of the HCP and FCC phases of the Fe lattice in addition to the $\mathrm{BCC}$ response already discussed above. Consider the HCP phase obtained by the phase transformation (unit cell shown in the inset of Fig. B.1). All atoms in the unit cell are displaced by $u_{i}=\left(F_{i j}-\delta_{i j}\right) X_{j}$, where $\delta_{i j}$ is the Kronecker delta, $X_{j}$ the positions of each of the atoms in the corresponding unstrained BCC unit cell and $F_{i j}$ as prescribed above. The resulting strain energy and $\Sigma_{22}-e_{22}$ responses of the HCP lattice are included in Figs. B.1a and B. $1 \mathrm{~b}$ respectively. Note that for the HCP lattice, $\Sigma_{22} \neq 0$ at $e_{22}=0$ because this strain is measured with respect to the unstrained BCC unit cell. Finally, we consider the FCC phase, i.e. $\gamma-$ Fe. We obtain this phase by restacking the HCP unit cell (the FCC unit cell is also shown in the inset of Fig. B.1) and the straining it as described above for the HCP phase. The $\Phi-e_{22}$ and $\Sigma_{22}-e_{22}$ predictions are included in Fig. B.1: this phase is energetically unfavourable in this orientation for all levels of imposed strain considered here.

\section{Appendix C: Ductile void growth}

There is an extensive literature on ductile void growth (Benzerga and Leblond, 2010) with the Gurson (1977) model as modified by Tvergaard and Needleman (1984) widely used. Here we shall use this classical approach to predict the evolution of $\eta$ although we emphasize that other more sophisticated void growth models (Danas and Aravas, 2012) can equally also be employed. In the Tvergaard and Needleman (1984) model, the yield surface for a voided metal in terms of co-efficients $q_{1}=1.5, q_{2}=1$ and $q_{3}=q_{1}^{2}$ is

$$
\Psi \equiv\left(\frac{\sigma_{\mathrm{e}}}{\bar{\sigma}}\right)^{2}+2 q_{1} \eta \cosh \left(\frac{3 q_{2} \sigma_{\mathrm{m}}}{2 \bar{\sigma}}\right)-1-q_{3} \eta^{2}=0,
$$

where under uniaxial tensile loading the von-Mises equivalent stress $\sigma_{\mathrm{e}}=\sigma^{\infty}$ and the mean stress $\sigma_{\mathrm{m}}=\sigma^{\infty} / 3$. The plastic strain-rate follows from the normality relation as $\dot{\varepsilon}_{i j}^{\mathrm{p}}=$ $\dot{\Lambda} \partial \Psi / \partial \sigma_{i j}$ in terms of the plastic multiplier $\dot{\Lambda}$. With plastic deformation of the metal assumed to be incompressible, the evolution of the void volume fraction is given by

$$
\frac{d \eta}{d \varepsilon_{k k}^{\mathrm{p}}}=(1-\eta)
$$

and for uniaxial tensile loading in the $x_{2}$-direction, the differential equation for the growth of $\eta$ in terms of the tensile plastic strain $\varepsilon_{22}^{\mathrm{p}}$ reads as

$$
\frac{d \eta}{d \varepsilon_{22}^{\mathrm{p}}}=\frac{(1-\eta)\left[\frac{3 q_{1} q_{2} \eta}{\bar{\sigma}} \sinh \left(\frac{q_{2} \sigma^{\infty}}{2 \bar{\sigma}}\right)\right]}{\frac{2 \sigma^{\infty}}{\bar{\sigma}^{2}}+\frac{q_{1} q_{2} \eta}{\bar{\sigma}} \sinh \left(\frac{q_{2} \sigma^{\infty}}{2 \bar{\sigma}}\right)}
$$

with initial conditions $\eta=\eta_{0}$ at $\varepsilon_{22}^{\mathrm{p}}=0$. Here $\bar{\sigma}\left(\varepsilon^{\mathrm{p}}\right)$ denotes the current flow strength at a microscopic equivalent plastic strain $\varepsilon^{\mathrm{p}}$ and is given by Eq. (3.1). This microscopic plastic 
strain $\varepsilon^{\mathrm{p}}$ is related to the macroscopic strain $\varepsilon_{22}^{\mathrm{p}}$ via a balance of macroscopic and microscopic plastic work, viz.

$$
\frac{d \varepsilon^{\mathrm{p}}}{d \varepsilon_{22}^{\mathrm{p}}}=\frac{\sigma^{\infty}}{(1-\eta) \bar{\sigma}},
$$

where the applied stress $\sigma^{\infty}$ satisfies the yield criterion (C.1) such that

$$
\left(\frac{\sigma^{\infty}}{\bar{\sigma}}\right)^{2}+2 q_{1} \eta \cosh \left(\frac{q_{2} \sigma^{\infty}}{2 \bar{\sigma}}\right)-1-q_{3} \eta^{2}=0
$$

The solution of the differential equation (C.4) with initial conditions $\varepsilon^{\mathrm{p}}=0$ at $\varepsilon_{22}^{\mathrm{p}}=0$, together with (C.3) then yields the void fraction $\eta$.

\section{Acknowledgements}

SSS and GC acknowledge EPSRC grant EP/L014742/1. VSD acknowledges the insightful discussions that arose at the workshop on environmental effects on fracture held by the Department of Materials Science and Engineering at Texas A\&M University and organised by Prof. Alan Needleman. The authors are also grateful to Prof. William Curtin (EPFL) for insightful comments, discussions and feedback.

\section{References}

Andric, P., Curtin, W.A., 2019. Atomistic modelling of fracture. Model. Simul. Mat. Sci. Eng. $27,013001$.

Armstrong, R.W., Arnold, W., Zerilli, F.J., 2009. Dislocation mechanics of copper and iron in high rate deformation tests. J. App. Phys. 105, 023511.

Ayas, C., Deshpande, V.S., Fleck, N.A., 2014. A fracture criterion for the notch strength of high strength steels in the presence of hydrogen. J. Mech. Phys. Solids 63, 80-93.

Ayas, C., Deshpande, V.S., Fleck, N.A., 2015. Hydrogen embrittlement of a bimaterial. Mech. Mater. 80, 193-202.

Barenblatt, G.I., 1962. The mathematical theory of equilibrium cracks in brittle fracture. Adv. Appl. Mech. 7, 55-129.

Beachem, C.D., 1972. A new model for hydrogen-assisted cracking (hydrogen 'embrittlement'). Metall. Trans. 3 (2), 437-451.

Bechtle, S., Kumar, M., Somerday, B.P., Launey, M.E., Ritchie, R.O., 2009. Grain-boundary engineering markedly reduces susceptibility to intergranular hydrogen embrittlement in metallic materials, Acta Mater. 57, 4148-4157.

Benzerga, A.A., Leblond, J.B., 2010. Ductile fracture by void growth to coalescence. Adv. App. Mech. 44, 169-305

Bhadeshia, H.K.D.H., 2016. Prevention of hydrogen embrittlement in steels. ISIJ Int. 56, 24 36.

Birnbaum, H., Sofronis, P., 1994. Hydrogen-enhanced localized plasticity a mechanism for hydrogen-related fracture. Mater. Sci. Eng.: A 176 (1-2), 191-202.

Brahimi, S. V., Yue, S., and Sriraman, K.R., 2017. Alloy and composition dependence of hydrogen embrittlement susceptibility in high-strength steel fasteners. Phil. Trans. R. Soc. A $375,20160407$. 
Dadfarnia, M., Nagao, A., Wang, S., Martin, M. L., Somerday, B.P., Sofronis, P., 2015. Recent advances on hydrogen embrittlement of structural materials. Int. J. Fract. 196, 223-243.

Danas, K., Aravas, N., 2012. Numerical modeling of elasto-plastic porous materials with voi shape effects at finite deformations. Composites Part B: Engineering 43, 2544-2559.

Dugdale, D.S., 1960. Yielding of steel sheet containing slits. J. Mech. Phys. Solids 8, 100-104.

François, D., Pineau, A., Zaoui, A., 2012. Mechanical behavior of materials: volume II: fracture mechanics and damage, vol. 191. Springer, Berlin.

Freund, L.B., Hutchinson, J.W., 1985. High strain-rate crack growth in rate-dependent plastic solids. J. Mech. Phys. Solids 33, 169-191.

Fujita, S., Murakami, Y., 2013. A new nonmetallic inclusion rating method by positive use of hydrogen embrittlement phenomenon. Metall. Mater. Trans. A Phys. Metall. Mater. Sci. 44, $303-322$.

Gangloff, R.P., 2003. Hydrogen assisted cracking of high strength alloys. In: Milne, I., Ritchie, R.O., Karihaloo, B. (Eds.), Comprehensive Structural Integrity, vol. 6. Elsevier Science, New York, NY, 31-101 (J. Petit and P. Scott, Volume Editors).

Giovanola, J.H., Cannizzaro, D., Doglione, R., Rossoll A., 2006. Ductile fracture by void nucleation at tempering carbides. E.E. Gdoutos (Ed.), Fracture of Nano and Engineering Materials and Structures, Springer.

Gurson, A.L., 1977. Continuum theory of ductile rupture by void nucleation and growth: path I- Yield function and flow rules for porous ductile media. ASME Transaction, J. Eng. Mat. Tech. 99, 2-17.

Hahn, G.T., 1984. The influence of microstructure on brittle fracture toughness. Metall. Trans. A 15A, 947-959.

Henkelman, G., Jónsson, H., 2000. Improved tangent estimate in the nudged elastic band method for finding minimum energy paths and saddle points. J. Chem. Phys. 113, 9978-9985.

Hirth, J. P., 1980. Effects of hydrogen on the properties of iron and steel. Metall. Trans. A 11(6), 861-890.

Jiang, D.E., Carter, E.A., 2004a. Diffusion of interstitial hydrogen into and through bcc Fe from first principles. Phys. Rev. B., 70, 64102-64110.

Jiang, D.E., Carter, E.A., 2004b. First principles assessment of ideal fracture energies of materials with mobile impurities: implications for hydrogen embrittlement of metals. Acta Mater. 52, 4801-4807.

Johnson, W.H., 1875. On some remarkable changes produced in iron and steel by the action of hydrogen and acids. Proc. Roy. Soc. 23, 168-179.

Kameda, J., McMahon, C.J., 1983. Solute segregation and hydrogen-induced intergranular fracture in an alloy steel, Mettal. Trans. A 14, 903-911.

Kroon, M., Faleskog, J., 2005. Micromechanics of cleavage fracture initiation in ferritic steels by carbide cracking, J. Mech. Phys. Solids 53, 171-196.

Landis, C.M., Pardoen, T., Hutchinson, J.W., 2000. Crack velocity dependent toughness in rate dependent materials. Mech. Mater. 32, 663-678.

Li, D., Gangloff, R., Scully, J., 2004. Hydrogen trap states in ultrahigh-strength AERMET 100 steel. Metall. Mater. Trans. A 35, 849-864. 
Li, X., Zhang, J., Shen, S., Wang, Y., Song, X., 2017. Effect of tempering temperature and inclusions on hydrogen-assisted fracture behaviors of a low alloy steel. Mater. Sci. Eng. A 682, 359-369.

Lynch, S.P., 2013. Mechanisms and kinetics of environmentally assisted cracking: current status, issues, and suggestions for further work. Metallur. Mater. Trans. A-Phys. Metallur. Mater. Sci., 44A, 1209-1229.

Majumdar, B.S., Burns, S.J., 1981. Crack-tip shielding — an elastic theory of dislocations and dislocation arrays near a sharp crack. Acta Metall. 29 (4), 579-588.

Martin, M.L., Dadfarnia, M., Nagao, A., Wang, S., Sofronis, P., 2019. Enumeration of the hydrogen-enhanced localized plasticity mechanism for hydrogen embrittlement in structural materials. Acta Mater. 165, 734-750.

Martínez-Pañeda, E., Niordson, C.F., Gangloff, R.P., 2016. Strain gradient plasticity-based modeling of hydrogen environment assisted cracking. Acta Mater. 117, 321-332.

McClintock, F.A., 1968. A criterion for ductile fracture by the growth of holes. J. Appl. Mech., 35, 363-371.

McMeeking, R.M., Parks, D.M., 1979. On criterion for J-dominance of crack tip fields in large scale yielding. ASTM Spec. Tech. Publ. 668, 175-194.

Merrill, P.B., Madix, R.J., 1996. Hydrogen bonding on iron: correlation of adsorption and desorption states on $\mathrm{Fe}(100)$ and perturbation of the $\mathrm{Fe}-\mathrm{H}$ bond with coadsorbed $\mathrm{CO}$. Surface Sci. 347, 249-264.

Nagao, A., Dadfarnia, M., Somerday, B.P., Sofronis, P., Ritchie, R.O., 2018. Hydrogenenhanced-plasticity mediated decohesion for hydrogen-induced intergranular and quasicleavage fracture of lath martensitic steels. J. Mech. Phys. Solids 112, 403-430.

Nagao, A., Smith, C.D., Dadfarnia, M., Sofronis, P., Robertson, I.M., 2012. The role of hydrogen in hydrogen embrittlement fracture of lath martensitic steel. Acta Mater. 60, 51825189.

Nelson, H.G., Stein, J.E., 1973. Gas-phase hydrogen permeation through alpha iron, 4130 steel, and 304 stainless steel from less than 100 to near 6001C. NASA Technical Note D-7265.

Novak, P., Yuan, R., Somerday, B.P., Sofronis, P., Ritchie, R.O., 2010. A statistical, physicalbased, micro-mechanical model of hydrogen-induced intergranular fracture in steel. J. Mech. Phys. Solids 58 (2), 206-226.

Oriani, R.A., 1970. The diffusion and trapping of hydrogen in steel. Acta Metall. 18 (1), 147157.

Oriani, R.A., 1972. Mechanistic theory of hydrogen embrittlement of steels. Berichte BunsenGes. Phys. Chem. 76 (8), 848-857.

Pfeil, L.P., 1926. The effect of occluded hydrogen on the tensile strength of iron. Proc. Roy. Soc. $112,182-195$.

Rajan, V.P., Curtin, W.A., 2016. Crack tip blunting and cleavage under dynamic conditions. J. Mech. Phys. Solids 90, 18-28.

Ramasubramaniam, A., Itakura M., Carter E.A., 2009. Interatomic potentials for hydrogen in $\alpha$-iron based on density functional theory. Phys Rev B 79, 174101.

Rice, J.R., 1968. A path independent integral and the approximate analysis of strain concentration by notches and cracks. J. App. Mech. 35 (2), 379-386. 
San Marchi, C., Somerday, B.P., 2012. Technical reference for hydrogen compatibility of materials. Sandia National Laboratories, Albuquerque, New Mexico and Livermore, California. Report No.: SAND2012-7321.

San Marchi, C., Somerday, B.P., Robinson, S.L., 2007. Permeability, solubility and diffusivity of hydrogen isotopes in stainless steels at high gas pressures. Int. J. Hydrogen Energy 32, 100116.

Serebrinsky, S., Carter, E.A., Ortiz, M., 2004. A quantum-mechanically informed continuum model of hydrogen embrittlement. J. Mech. Phys. Solids 52 (10), 2403-2430.

Shibata, A., Murata, T., Takahashi, H., Matsuoka, T., Tsuji, N., 2016. Characterization of hydrogen-related fracture behavior in as-quenched low-carbon martensitic steel and tempered medium-carbon martensitic. Metall. Mater. Trans. A 46(12), 5685-5696.

Sih, G.C., Paris, P.C., Irwin, I.G., 1965. On cracks in rectilinearly anisotropic bodies. Int. J. Fract. Mech. 1, 189-203.

Sofronis, P., McMeeking, R.M., 1989. Numerical analysis of hydrogen transport near a blunting crack tip. J. Mech. Phys. Solids 37 (3), 317-350.

Song, J., Curtin, W.A., 2010. Testing continuum concepts for hydrogen embrittlement in metals using atomistics. Model. Simul. Mat. Sci. Eng. 18, 045003.

Song, J., Curtin, W.A., 2013. Atomic mechanism and prediction of hydrogen embrittlement in iron. Nat. Mater. 12, 145-151.

Sorescu, D.C., 2005. First principles calculations of the adsorption and diffusion of hydrogen on $\mathrm{Fe}(100)$ surface and in the bulk. Catalysis Today 105(1), 44-65.

Tehranchi, A., Curtin, W.A., 2017a. Atomistic study of hydrogen embrittlement of grain boundaries in nickel: I. fracture. J. Mech. Phys. Solids 101, 150-165.

Tehranchi, A., Curtin, W.A., 2017b. Atomistic study of hydrogen embrittlement of grain boundaries in nickel: II. decohesion. Model. Simul. Mat. Sci. Eng. 25(7), 075013.

Troiano, A.R., 1960. The role of hydrogen and other interstitials in the mechanical behaviour of metals. Trans. ASM 52, 54-80.

Tvergaard, V., Hutchinson, J.W., 1992. The relation between crack growth resistance and fracture process parameters in elastic-plastic solids. J. Mech. Phys. Solids 40, 1377-1397.

Tvergaard, V., Needleman, A., 1984. Analysis of the cup-cone fracture in a round tensile bar. Acta Metall. 32, 157-169.

Wang, S., Martin, M.L., Sofronis, P., Ohnuki, S., Hashimoto, N., Robertson, I.M., 2014. Hydrogen-induced intergranular failure of iron. Acta Mater. 69, 275-282.

Wang, J.-S., Vehoff, H., 1991. The effect of the mobility of segregated atoms on interfacial embrittlement. Scr. Metall. Mater. 25, 1339-1344.

Yahya, B., Pineau, P., 1998. Statistical modelling of intergranular brittle fracture in a low alloy steel. Fatigue Fract. Eng. Mater. Struct. 21 (12), 1485-1502.

Yamasaki, S., Bhadeshia, H., 2006. $\mathrm{M}_{4} \mathrm{C}_{3}$ precipitation in Fe-C-Mo-V steels and relationship to hydrogen trapping. Proc. R. Soc. A: Math. Phys. Eng. Sci. 462(2072), 2315-2330.

Zou, C., van Duin, A.C.T., Sorescu, D.C., 2012. Theoretical investigation of hydrogen adsorption and dissociation on iron and iron carbide surfaces using the ReaxFF reactive force field method. Topics in Catalysis, 55(5-6), 391-401. 